\title{
Atualização de Tópicos Emergentes da Diretriz Brasileira de Insuficiência Cardíaca - 2021
}

\section{Emerging Topics Update of the Brazilian Heart Failure Guideline - 2021}

Realização: Departamento de Insuficiência Cardíaca (DEIC) da Sociedade Brasileira de Cardiologia (SBC)

Conselho de Normatizações e Diretrizes (2020-2021): Antonio Carlos Sobral Sousa, Aurora Felice Castro Issa, Bruno Ramos Nascimento, Harry Corrêa Filho, Marcelo Luiz Campos Vieira

Coordenador de Normatizações e Diretrizes (2020-2021): Brivaldo Markman Filho

Autores da Diretriz: Fabiana G. Marcondes-Braga, ${ }^{\circledR}$ Lídia Ana Zytynski Moura, ${ }^{2}{ }^{\circledR}$ Victor Sarli Issa, ${ }^{3}$ Jefferson Luis Vieira, ${ }^{4}$ Luis Eduardo Rohde, ${ }^{5,6,7}$ Marcus Vinícius Simões, ${ }^{8}$ Miguel Morita Fernandes-Silva, ${ }^{9,10 \circlearrowright}$ Salvador Rassi, ${ }^{11}$ Silvia Marinho Martins Alves, ${ }^{12,13}$ Denilson Campos de Albuquerque, ${ }^{14}$ Dirceu Rodrigues de Almeida, ${ }^{15}$ Edimar Alcides Bocchi, ${ }^{1 \oplus}$ Felix José Alvarez Ramires, ${ }^{1,17}{ }^{\circledR}$ Fernando Bacal, ${ }^{1 \oplus}$ João Manoel Rossi Neto, ${ }^{18}$ Luiz Claudio Danzmann, ${ }^{19,20}$ Marcelo Westerlund Montera, ${ }^{21}$ Mucio Tavares de Oliveira Junior, ${ }^{10}$

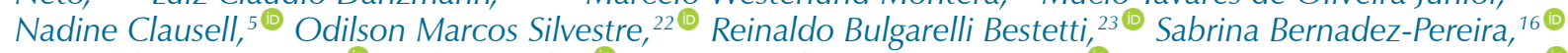
Aguinaldo F. Freitas Jr, ${ }^{11}$ Andréia Biolo, ${ }^{5}$ Antonio Carlos Pereira Barretto, ${ }^{1 \oplus}$ Antônio José Lagoeiro Jorge, ${ }^{24}{ }^{\circledR}$ Bruno Biselli, ${ }^{\circledR}$ Carlos Eduardo Lucena Montenegro, ${ }^{12,13 @ ~ E d v a l ~ G o m e s ~ d o s ~ S a n t o s ~ J u ́ n i o r, ~}{ }^{25,26}$ Estêvão Lanna

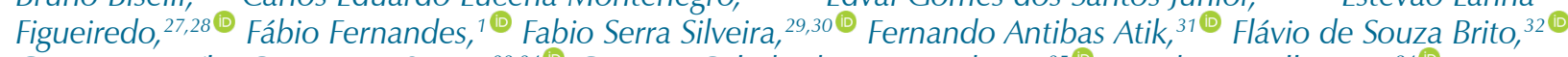

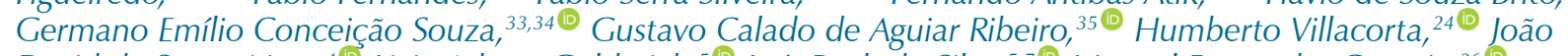
David de Souza Neto, ${ }^{4 \oplus}$ Livia Adams Goldraich, ${ }^{5}$ Luís Beck-da-Silva, ${ }^{5,7}$ Manoel Fernandes Canesin, ${ }^{36}{ }^{\oplus}$ Marcelo Imbroinise Bittencourt, $14,37 @$ Marcely Gimenes Bonatto, ${ }^{38}$ Maria da Consolação Vieira Moreira, ${ }^{39} \bullet$ Mônica Samuel Avila, ${ }^{1}$ Otavio Rizzi Coelho Filho, ${ }^{40}$ Pedro Vellosa Schwartzmann, ${ }^{41,42 @ ~ R i c a r d o ~ M o u r i l h e-~}$ Rocha, ${ }^{14 @}$ Sandrigo Mangini, ${ }^{\circledR}$ Silvia Moreira Ayub Ferreira, ${ }^{10}$ José Albuquerque de Figueiredo Neto, ${ }^{43}$ Evandro Tinoco Mesquita24,44인

Instituto do Coração (InCor) do Hospital das Clínicas da Faculdade de Medicina da Universidade de São Paulo (HCFMUSP), ${ }^{1}$ São Paulo, SP - Brasil Pontifícia Universidade Católica de Curitiba, ${ }^{2}$ Curitiba, $P R-$ Brasil

Universidade da Antuérpia, ${ }^{3}$ - Bélgica

Hospital do Coração de Messejana Dr. Carlos Alberto Studart Gomes, ${ }^{4}$ Fortaleza, CE - Brasil

Hospital de Clínicas de Porto Alegre, ${ }^{5}$ Porto Alege, RS - Brasil

Hospital Moinhos de Vento, ${ }^{6}$ Porto Alegre, RS - Brasil

Universidade Federal do Rio Grande do Sul (UFRGS), ${ }^{7}$ Porto Alegre, RS - Brasil

Faculdade de Medicina de Ribeirão Preto da Universidade de São Paulo, ${ }^{8}$ São Paulo, SP - Brasil

Universidade Federal do Paraná (UFPR), ${ }^{9}$ Curitiba, PR - Brasil

Quanta Diagnóstico por Imagem, ${ }^{10}$ Curitiba, PR - Brasil

Hospital das Clínicas da Universidade Federal de Goiás (UFCO), ${ }^{11}$ Goiânia, GO - Brasil

Pronto Socorro Cardiológico de Pernambuco (PROCAPE), ${ }^{12}$ Recife, PE - Brasil

Universidade de Pernambuco (UPE), ${ }^{13}$ Recife, PE - Brasil

Universidade do Estado do Rio de Janeiro (UERJ), ${ }^{14}$ Rio de Janeiro, RJ - Brasil

Universidade Federal de São Paulo (UNIFESP), ${ }^{15}$ São Paulo, SP - Brasil

Hospital de Coração (HCor), ${ }^{16}$ São Paulo, SP - Brasil

Hospital Israelita Albert Einstein, ${ }^{17}$ São Paulo, SP - Brasil

Instituto Dante Pazzanese de Cardiologia, ${ }^{18}$ São Paulo, SP - Brasil

Universidade Luterana do Brasil, ${ }^{19}$ Canoas, RS - Brasil

Hospital São Lucas da PUC-RS, ${ }^{20}$ Porto Alegre, RS - Brasil

Hospital Pró-Cardíaco, ${ }^{21}$ Rio de Janeiro, $R J-$ Brasil

Universidade Federal do Acre, ${ }^{22}$ Rio Branco, AC - Brasil

Departamento de Medicina da Universidade de Ribeirão Preto (UNAERP), ${ }^{23}$ Ribeirão Preto, SP - Brasil

Faculdade de Medicina da Universidade Federal Fluminense (UFF), ${ }^{24}$ Niterói, RJ - Brasil

Universidade Estadual de Feira de Santana, ${ }^{25}$ Feira de Santana, BA - Brasil

Santa Casa de Misericórdia de Feira de Santana, ${ }^{26}$ Feira de Santana, BA - Brasil 
Instituto Orizonti, ${ }^{27}$ Belo Horizonte, MG - Brasil

Hospital Vera Cruz, ${ }^{28}$ Belo Horizonte, MG - Brasil

Fundação Beneficência Hospital de Cirurgia (FBHC-Ebserh), ${ }^{29}$ Aracaju, SE - Brasil

Centro de Pesquisa Clínica do Coração, ${ }^{30}$ Aracaju, SE - Brasil

Universidade de Brasília (UnB), ${ }^{31}$ Brasília, DF - Brasil

Universidade Estadual Paulista Júlio de Mesquita Filho (UNESP), ${ }^{32}$ São Paulo, SP - Brasil

Hospital Alemão Oswaldo Cruz, ${ }^{33}$ São Paulo, SP - Brasil

Hospital Regional de São José dos Campos, ${ }^{34}$ São Paulo, SP - Brasil

Pontifícia Universidade Católica de Campinas (PUCC), ${ }^{35}$ Campinas, SP - Brasil

Hospital Universitário da Universidade Estadual de Londrina, ${ }^{36}$ Londrina, PR - Brasil

Hospital Universitário Pedro Ernesto, ${ }^{37}$ Rio de Janeiro, RJ - Brasil

Hospital Santa Casa de Misericórdia de Curitiba, ${ }^{38}$ Curitiba, PR - Brasil

Universidade Federal de Minas Gerais (UFMG), ${ }^{39}$ Belo Horizonte, MG - Brasil

Faculdade de Ciências Médicas da Universidade Estadual de Campinas (UNICAMP), ${ }^{40}$ Campinas, SP - Brasil

Hospital Unimed Ribeirão Preto, ${ }^{41}$ Ribeirão Preto, SP - Brasil

Centro Avançado de Pesquisa, Ensino e Diagnóstico (CAPED), ${ }^{42}$ Ribeirão Preto, SP - Brasil

Universidade Federal do Maranhão (UFMA), ${ }^{43}$ São Luís, MA - Brasil

Centro de Ensino e Treinamento Edson de Godoy Bueno / UHG, ${ }^{44}$ Rio de Janeiro, RJ - Brasil

Comitê Editor da Diretriz: Fabiana Marcondes-Braga, Lídia Ana Zytynski Moura, Victor Sarli Issa, José Albuquerque Figueiredo Neto, Evandro Tinoco Mesquita

Comitê Revisor da Diretriz: Fabiana Marcondes-Braga, Lídia Ana Zytynski Moura, Victor Sarli Issa, Jefferson Luis Vieira, Luis Eduardo Rohde, Marcus Vinícius Simões, Miguel Morita Fernandes-Silva, Salvador Rassi, Silvia Marinho Martins Alves, José Albuquerque de Figueiredo Neto, Evandro Tinoco Mesquita

Coordenadores de Grupos de Discussão: Denilson Campos de Albuquerque, Dirceu Rodrigues de Almeida, Edimar Alcides Bocchi, Felix José Alvarez Ramires, Fernando Bacal, João Manoel Rossi Neto, Luiz Claudio Danzmann, Luis Eduardo Rohde, Marcelo Westerlund Montera, Marcus Vinicius Simões, Miguel Morita Fernandes- Silva, Mucio Tavares de Oliveira Junior, Nadine Clausell, Odilson Marcos Silvestre, Reinaldo Bulgarelli Bestetti, Sabrina Bernadez-Pereira, Salvador Rassi, Silvia Marinho Martins

Demais Colaboradores: Aguinaldo F. Freitas Jr, Andréia Biolo, Antonio Carlos Pereira Barretto, Antônio José Lagoeiro Jorge, Bruno Biselli, Carlos Eduardo Lucena Montenegro, Edval Gomes dos Santos Júnior, Estêvão Lanna Figueiredo, Fábio Fernandes, Fabio Serra Silveira, Fernando Antibas Atik, Flávio de Souza Brito, Germano Emílio Conceição Souza, Gustavo Calado de Aguiar Ribeiro, Humberto Villacorta, João David de Souza Neto, Livia Adams Goldraich, Luís Beck-da-Silva Neto, Manoel Fernandes Canesin, Marcelo Bittencourt, Marcely Bonatto, Maria da Consolação Vieira Moreira, Mônica Samuel Avila, Otavio Rizzi Coelho Filho, Pedro Vellosa Schwartzmann, Ricardo Mourilhe-Rocha, Sandrigo Mangini, Silvia Moreira Ayub Ferreira

Esta diretriz deve ser citada como: Marcondes-Braga FG, Moura LAZ, Issa VS, Vieira JL, Rohde LE, Simões MV, et al. Atualização de Tópicos Emergentes da Diretriz de Insuficiência Cardíaca - 2021. Arq Bras Cardiol. 2021; 116(6):1174-1212

Nota: Estas atualizações se prestam a informar e não a substituir o julgamento clínico do médico que, em última análise, deve determinar o tratamento apropriado para seus pacientes.

Correspondência: Sociedade Brasileira de Cardiologia - Av. Marechal Câmara, 360/330 - Centro - Rio de Janeiro-CEP: 20020-907. E-mail: diretrizes@cardiol.br 


\section{Atualização}

\section{Sumário}

Introdução 1177

1. Inovações em Insuficiência Cardíaca com Fração de Ejeção Preservada (ICFEp), Levemente Reduzida (ICFEIr) e Melhorada (ICFEm)

1177

1.1. Diagnóstico de Insuficiência Cardíaca com Fração de Ejeção

Preservada (ICFEp)

1.2. Tratamento da Insuficiência Cardíaca com Fração de Ejeção

Preservada (ICFEp)

1.3. Tratamento da Insuficiência Cardíaca com Fração de Ejeção

Levemente Reduzida (ICFEIr)

1.4. Tratamento da Insuficiência Cardíaca com Fração de Ejeção Melhorada (ICFEm) 1181

2. Inovações em Amiloidose Cardíaca ............................... 1181

2.1. Quando Suspeitar de Amiloidose .............................................. 1181

2.2. Diagnóstico de Amiloidose Cardíaca ............................................ 1182

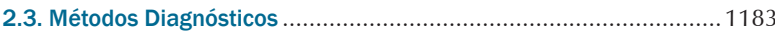

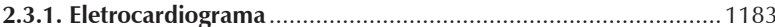

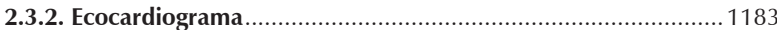

2.3.3. Cintilografia Cardíaca com Radiotraçadores Ósseos............... 1183

2.3.4. Ressonância Magnética Cardíaca ............................................... 1183

2.4. Tratamento de Amiloidose Cardíaca por Transtirretina (AC-ATTR) ... 1183

3. Inovações em Telemonitoramento na Insuficiência Cardíaca

1184

4. Inovações em Cardiointervenção ................................... 1184

4.1. Abordagem Percutânea da Insuficiência Mitral Secundária........... 1184

4.2. Ablação de Fibrilação Atrial............................................................ 1185

5. COVID-19 e a Insuficiência Cardíaca............................... 1185

6. Inovações em Insuficiência Cardíaca Avançada..........1186

6.1. Definição de Insuficiência Cardíaca Avançada...............................1186

6.2. Papel do Especialista em Insuficiência Cardíaca Avançada .......... 1188

6.3. Abordagem do Paciente com Insuficiência Cardíaca Avançada.... 1188
6.4. Inovações sobre o Manejo da Congestão em Pacientes com

Insuficiência Cardíaca Avançada ......................................................... 1189

6.5. Classificação Atual de Choque Cardiogênico ................................1190

6.6. Aplicabilidade do Cateter de Artéria Pulmonar na Insuficiência

Cardíaca Avançada.......................................................................... 1192

6.7. Inovações com Relação aos Dispositivos de Assistência Circulatória de

Curta Duração na Insuficiência Cardíaca Avançada.

.. 1192

6.8. Inovações com Relação aos Cuidados Paliativos na Insuficiência

Cardíaca Avançada.............................................................................. 1193

7. Tratamento da Insuficiência Cardíaca com Fração de

Ejeção Reduzida (ICFEr) .......................................................... 1194

7.1. Estratégias Farmacológicas Previamente Consolidadas para

Tratamento da Insuficiência Cardíaca com Fração de Ejeção Reduzida

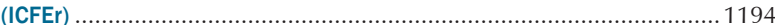

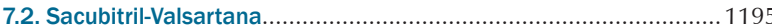

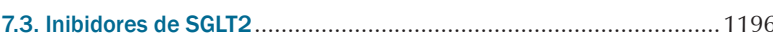

7.4. Tratamento de Comorbidades na Insuficiência Cardíaca com Fração de Ejeção Reduzida ............................................................................. 1197

7.4.1. Diabetes Tipo 2 …............................................................ 1197

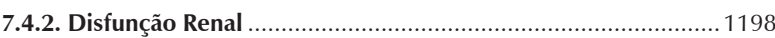

7.4.3. Deficiência de Ferro ................................................................... 1198

7.5. Algoritmo de Tratamento da Insuficiência Cardíaca com Fração de

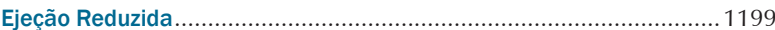

8. Inovações em Outros Temas Relacionados à Insuficiência Cardíaca

8.1. Biomarcadores na Insuficiência Cardíaca com Fração de Ejeção

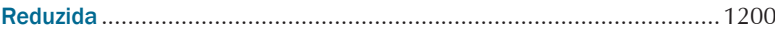

8.2. Imunizações na Insuficiência Cardíaca......................................... 1201

8.3. Indicação de Avaliação Genética nas Cardiomiopatias e na Insuficiência Cardíaca .

9. Perspectivas na Insuficiência Cardíaca - Novas

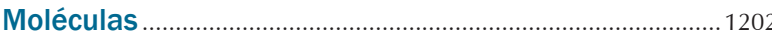

9.1. Estimuladores da Guanilato Ciclase ............................................ 1202

9.2. Ativador Seletivo da Miosina Cardíaca...................................... 1202

Referências..................................................................... 1203 


\section{Introdução}

A última Diretriz de Insuficiência Cardíaca do Departamento de Insuficiência Cardíaca da Sociedade Brasileira de Cardiologia (DEIC/SBC) foi finalizada em março de 2018. A partir de então, houve um importante número de intervenções terapêuticas e abordagens diagnósticas que surgiram ou se consolidaram na prática clínica internacional e na pesquisa clínica. Ao lado disso, a pandemia da COVID-19 trouxe-nos conhecimento sobre o modelo fisiopatológico da agressão miocárdica e muitas dúvidas acerca da continuidade e da segurança dos medicamentos nos pacientes com IC crônica que apresentaram quadro agudo dessa complexa e nova entidade clínica.

Nos últimos 6 meses, trabalhamos de forma rápida e colaborativa utilizando pela primeira vez em 20 anos do DEIC as plataformas digitais para discutir, deliberar e redigir esse importante documento, optando por realizar uma revisão focada em vez de uma ampla atualização da Diretriz ainda muito recente.

Inspiramo-nos no modelo de atualização da Diretriz Canadense de Insuficiência Cardíaca de 2020, ${ }^{1}$ porém tivemos a nosso favor a oportunidade de observar os impactos na prática clínica e da consolidação daquelas novidades, além de ter publicado novos resultados de ensaios clínicos dos últimos 12 meses. Para apresentar esses avanços, realizamos um pioneiro encontro científico em 19 de setembro de 2020, o I Heart Failure Summit Brazil 2020 (digital), com cerca de 900 participantes, muitos destes associados do DEIC.

A liderança da Diretoria Científica foi fundamental para a organização de diferentes grupos de trabalhos e elaboração de uma forma prática e segura de discussão e votação. Garantindo o distanciamento social e empregando a tecnologia digital, o encontro permitiu amplos debates sobre os diferentes pontos de vista alicerçados nas melhores evidências científicas.

No presente documento, o DEIC/SBC apresenta uma revisão e uma atualização detalhadas de sua Diretriz de Insuficiência Cardíaca Crônica. Os trabalhos tiveram início no mês de julho de 2020, com a definição do Comitê Editor, que estabeleceu prioridades, dividiu os 52 participantes em grupos de trabalho e definiu o cronograma das atividades. Estes grupos, compostos por cinco a sete participantes cada, deram início a intensas discussões virtuais que culminaram com a redação de tabelas preliminares, sendo posteriormente amplamente divulgadas e revisadas pelo Comitê Revisor composto por 11 membros. As discussões finais foram realizadas em plenária virtual em 4 de dezembro de 2020, com a participação de todos os colaboradores, nas quais as principais recomendações foram votadas. As decisões quanto às classes de recomendação foram definidas com a concordância de mais de $75 \%$ dos participantes.

As definições de Classes de Recomendação e Nível de Evidência respeitam as mesmas normas da última diretriz, conforme preconiza o SBC/CONDir para elaboração de diretrizes e são assim descritas:

\begin{tabular}{ll}
\hline Classes de Recomendação \\
\hline Classe I & $\begin{array}{l}\text { Condições para as quais há evidências conclusivas ou, em } \\
\text { sua falta, consenso geral de que o procedimento é seguro } \\
\text { e útil/eficaz }\end{array}$ \\
\hline Classe II & $\begin{array}{l}\text { Condições para as quais há evidências conflitantes e/ou } \\
\text { divergência de opinião sobre segurança, e utilidade/eficácia } \\
\text { do procedimento }\end{array}$ \\
\hline Classe IIA & $\begin{array}{l}\text { Peso ou evidência/opinião a favor do procedimento. A } \\
\text { maioria aprova }\end{array}$ \\
\hline Classe IIB & $\begin{array}{l}\text { Segurança e utilidade/eficácia menos bem estabelecida, } \\
\text { não havendo predomínio de opiniões a favor }\end{array}$ \\
\hline Classe III & $\begin{array}{l}\text { Condições para as quais há evidências e/ou consenso de } \\
\text { que o procedimento não é útil/eficaz e, em alguns casos, } \\
\text { pode ser prejudicial }\end{array}$ \\
\hline Níveis de Evidência \\
\hline Nível A & $\begin{array}{l}\text { Dados obtidos a partir de múltiplos estudos randomizados } \\
\text { de bom porte, concordantes e/ou de metanálise robusta de } \\
\text { estudos clínicos randomizados }\end{array}$ \\
\hline Nível B & $\begin{array}{l}\text { Dados obtidos a partir de metanálise menos robusta, a } \\
\text { partir de um único estudo randomizado ou de estudos não } \\
\text { randomizados (observacionais) }\end{array}$ \\
\hline Nível C & Dados obtidos de opiniões consensuais de especialistas \\
\hline
\end{tabular}

As recomendações terapêuticas propostas no presente documento embasam-se nas evidências científicas mais atuais, considerando não apenas os aspectos de eficácia clínica demonstrados em grandes ensaios clínicos. Buscamos sumarizar as principais recomendações em fluxogramas e algoritmos de fácil entendimento e grande aplicabilidade clínica, propondo abordagens para o diagnóstico e o tratamento da insuficiência cardíaca.

Nosso compromisso com a comunidade científica, ligado à pesquisa e à assistência aos pacientes com insuficiência cardíaca, gestores públicos e privados e também formuladores de políticas públicas, certamente contará com um documento que buscou apresentar as intervenções científicas de forma didática e, assim, facilitar sua implantação nas diferentes esferas de atendimento do paciente com insuficiência cardíaca.

Dr. Evandro Tinoco Mesquita

\section{Inovações em Insuficiência Cardíaca com Fração de Ejeção Preservada (ICFEp), Levemente Reduzida (ICFEIr) e Melhorada (ICFEm)}

\subsection{Diagnóstico de Insuficiência Cardíaca com Fração de Ejeção Preservada (ICFEp)}

No paciente com dispneia ou fadiga inexplicada, a avaliação da probabilidade pré-teste para insuficiência cardíaca (IC) deve ser efetuada com dados clínicos, eletrocardiográficos, ecocardiográfico e laboratorial. A seguir, na figura 1.1, os dois sistemas de escores desenvolvidos para confirmação deste diagnóstico: tanto a pontuação $\mathrm{H}_{2}$ FPEF (esquerda) quanto a HFA-PEFF (direita) podem ser utilizadas (Tabelas 


\section{Atualização}

1.1 e 1.2). Nesses modelos, os pacientes considerados de alta e baixa probabilidade são considerados como tendo ou não insuficiência cardíaca com fração de ejeção preservada (ICFEp), respectivamente. Nos pacientes com probabilidade intermediária, a avaliação da função diastólica durante estresse, que pode ser realizado por meio de teste hemodinâmico invasivo ou ecocardiografia de estresse diastólico, é capaz de auxiliar no diagnóstico de ICFEp. Nos pacientes com baixa probabilidade para a ICFEp, recomenda-se a investigação de outras causas de dispneia e fadiga ${ }^{2}$ (Figura 1.1 e Tabela 1.3).

\section{Insuficiência Cardiaca com Fração de Ejeção Preservada}

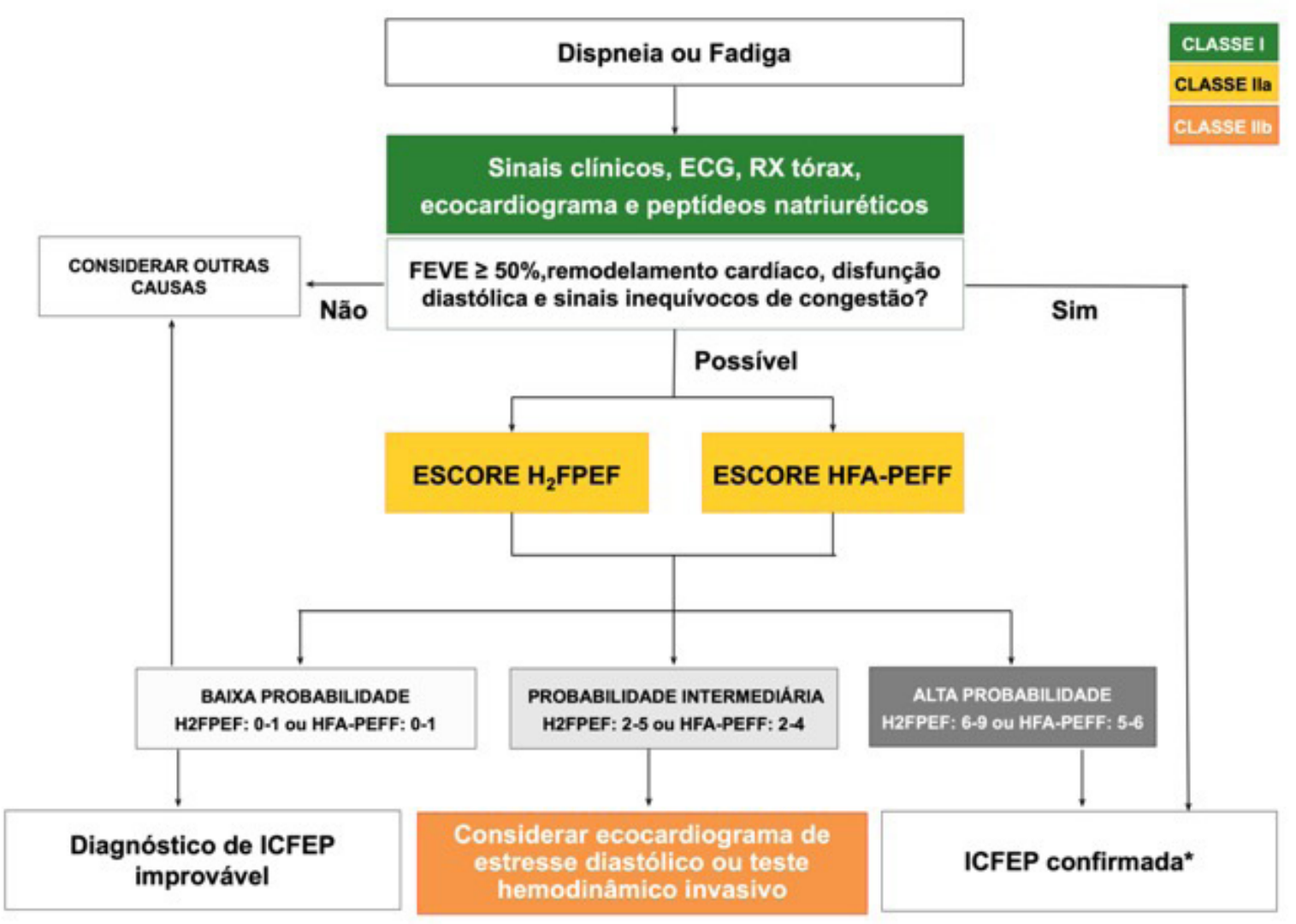

Figura 1.1 - Fluxograma diagnóstico de insuficiência cardiaca com fração de ejeção preservada (ICFEp)

Adaptado de: Borlaug BA. ${ }^{2}$ Nat Rev Cardiol. 2020; 17:559-573. ECG: eletrocardiograma; FEVE: fração de ejeção preservada; IC: insuficiência cardíaca.

Tabela 1.1 - Escore $\mathrm{H}_{2}$ FPEF para o diagnóstico da insuficiência cardiaca com fração de ejeção preservada (ICFEp)

\begin{tabular}{|c|c|c|c|}
\hline & Variável Clínica & Características & Pontos \\
\hline \multirow{2}{*}{$\mathrm{H}_{2}$} & Obesidade (Heavy) & $\mathrm{IMC}>30 \mathrm{Kg} / \mathrm{m}^{2}$ & 2 \\
\hline & Hipertensão & 2 ou mais anti-hipertensivos & 1 \\
\hline $\mathrm{F}$ & Fibrilação atrial & Paroxística ou persistente & 3 \\
\hline$P$ & Hipertensão Pulmonar & $\begin{array}{l}\text { PSAP > } 35 \mathrm{mmHg} \\
\text { (ecocardiograma) }\end{array}$ & 1 \\
\hline $\mathrm{E}$ & Idade avançada (E/derly) & Idade $>60$ anos & 1 \\
\hline $\mathrm{F}$ & Pressões de enchimento (Filling pressures) & $E / e^{\prime}>9$ & 1 \\
\hline
\end{tabular}

Adaptado de Reddy YNV et al. ${ }^{5}$ Circulation. 2018; 138:861-870. IMC: índice de massa corpórea; PSAP: pressão sistólica da artéria pulmonar. 


\begin{tabular}{|c|c|c|}
\hline CRITÉRIOS & $\begin{array}{c}\text { MAIOR } \\
\text { (2 Pontos) } \\
\end{array}$ & $\begin{array}{c}\text { MENOR } \\
\text { (1 ponto) }\end{array}$ \\
\hline FUNCIONAL & $\begin{array}{c}\text { e' septal }<7 \text { ou } \\
\text { e' lateral }<10 \text { ou } \\
\text { E/e' }>15 \text { ou } \\
\text { Velocidade RT }>2,8 \mathrm{~m} / \mathrm{s} \\
(\text { PSAP }>35 \mathrm{mmHg})\end{array}$ & $\begin{array}{l}\text { E/e' } 9-14 \text { ou } \\
\text { GLS }<16 \%\end{array}$ \\
\hline MORFOLÓGICO & $\begin{array}{c}\text { VAEI }>34 \mathrm{~mL} / \mathrm{m}^{2} \text { ou } \\
\text { Massa VE . } 149 / 122 \mathrm{~g} / \mathrm{m}^{2}(\mathrm{H} / \mathrm{M}) \text { e } \\
\text { ERP }>0,42\end{array}$ & $\begin{array}{c}\text { VAEi } 29-34 \mathrm{~mL} / \mathrm{m}^{2} \text { ou } \\
\text { Massa VE }>115 / 95 \mathrm{~g} / \mathrm{m}^{2}(\mathrm{H} / \mathrm{M}) \text { ou } \\
\text { ERP }>0,42 \text { ou } \\
\text { Septo ou } P P \geq 12 \mathrm{~mm}\end{array}$ \\
\hline $\begin{array}{l}\text { BIOMARCADOR } \\
\text { (Ritmo sinusal) }\end{array}$ & $\begin{array}{c}\text { NT-proBNP }>220 \mathrm{pg} / \mathrm{mL} \text { ou } \\
\text { BNP }>80 \mathrm{pg} / \mathrm{mL}\end{array}$ & $\begin{array}{c}\text { NT-proBNP } 125-220 \mathrm{pg} / \mathrm{mL} \text { ou } \\
\text { BNP } 35-80 \mathrm{pg} / \mathrm{mL}\end{array}$ \\
\hline $\begin{array}{l}\text { BIOMARCADOR } \\
\text { (Fibrilação atrial) }\end{array}$ & $\begin{array}{c}\text { NT-proBNP }>660 \mathrm{pg} / \mathrm{mL} \text { ou } \\
\text { BNP }>240 \mathrm{pg} / \mathrm{mL}\end{array}$ & $\begin{array}{l}\text { NT-proBNP } 365-660 \mathrm{pg} / \mathrm{mL} \text { ou } \\
\text { BNP } 105-240 \mathrm{pg} / \mathrm{mL}\end{array}$ \\
\hline
\end{tabular}

Adaptado de Pieske B et al. ${ }^{7}$ Heart Failure Association (HFA) of the European Society of Cardiology (ESC). Eur J Heart Fail. 2020; 22:391-412.

Velocidade RT: velocidade do fluxo de regurgitação da valva tricúspide; GLS: strain global longitudinal; VAEi: índice de volume atrial esquerdo; BNP: peptídeo natriurético do tipo B; NT-proBNP: peptídeo natriurético N-terminal pró-tipo B; VE: ventricular esquerda; H: homens / M:mulheres; ERP: espessura relativa da parede; PP: parede posterior.

Tabela 1.3 - Recomendações para o diagnóstico de insuficiência cardíaca com fração de ejeção preservada (ICFEp)

\begin{tabular}{|c|c|c|c|c|c|}
\hline Recomendações & Classe & NE & Comentários & $\begin{array}{c}\text { Tabela } \\
2018\end{array}$ & Ref. \\
\hline Peptídeos natriuréticos para rastreamento de ICFEp. & I & $\mathrm{B}$ & $\begin{array}{l}\text { NOVA: Devem ser consideradas a ampla variação do nível } \\
\text { sérico dos peptídeos natriuréticos nessa população e as } \\
\text { condições que modificam sua acurácia, como a fibrilação } \\
\text { atrial e a obesidade. }\end{array}$ & Nova & 3,4 \\
\hline $\begin{array}{l}\text { Ecocardiograma completo para confirmação do } \\
\text { diagnóstico. }\end{array}$ & I & $\mathrm{B}$ & $\begin{array}{l}\text { NOVA: Exame com apresentação de índices de Doppler } \\
\text { para estimativa de pressões diastólica e pulmonar, bem } \\
\text { como índices de volume e massa cardíaca indexados à } \\
\text { superfície corporal. }\end{array}$ & Nova & 4,5 \\
\hline $\begin{array}{l}\text { Escore diagnósticos } \mathrm{H}_{2} \text { FPF ou HFA PEFF para melhorar } \\
\text { a acurácia diagnóstica para ICFEp em pacientes com } \\
\text { suspeita clínica. }\end{array}$ & Ila & B & NOVA: Escores com validação em coortes retrospectivas. & Nova & $6-8$ \\
\hline $\begin{array}{l}\text { Avaliação durante estresse da função diastólica por } \\
\text { ecocardiografia ou avaliação hemodinâmica invasiva } \\
\text { em caso de dúvida diagnóstica após a aplicação dos } \\
\text { escores } \mathrm{H}_{2} \text { FPF ou HFA PEFF. }\end{array}$ & $\mathrm{Ilb}$ & B & NOVA: Escores com validação em coortes retrospectivas. & Nova & 9,10 \\
\hline
\end{tabular}

A estratégia inicial para o diagnóstico de ICFEp é a determinação da probabilidade pré-teste para IC, através do uso de achados clínicos associados a exames complementares como: eletrocardiograma, radiografia do tórax, ecocardiograma e peptídeos natriuréticos, se disponíveis. Para a interpretação do resultado dos peptídeos natriuréticos, é importante considerar que há grande variação dos níveis séricos nessa população e que, na vigência de fibrilação atrial (FA), devemos considerar pontos de corte mais elevados. ${ }^{3,4} \mathrm{Na}$ presença de plausibilidade para IC, é razoável a aplicação dos escores $\mathrm{H}_{2}$ FPEF5,6 (com dados clínicos e ecocardiográficos) e HFA PEFF (com dados ecocardiográficos completos e de peptídeos natriuréticos), os quais já foram validados em populações externas. ${ }^{6,8}$ para determinar a probabilidade alta, intermediária e baixa. Em pacientes com baixa probabilidade de ICFEp, sugere-se a busca objetiva para outras etiologias para dispneia. Nos indivíduos com probabilidade intermediária, estudos recentes demonstram que a observação dos dados diastólicos sob estresse físico pode revelar pacientes com resposta anormal, constituindo assim uma estratégia diagnóstica que pode ser não invasiva (observação diastólica por ecocardiograma) ${ }^{9}$ ou invasiva (cateterização da artéria pulmonar). ${ }^{10}$ Para a realização dos escores supracitados, faz-se necessária a realização do ecocardiograma completo, ou seja, exame com a extração dos diâmetros, índices de volume do átrio esquerdo e Doppler de fluxo, Doppler tecidual (e' septal e ou lateral) e, se possível, com dados da deformação miocárdica (strain e strain rate). ${ }^{5}$

FA: fibrilação atrial; IC: insuficiência cardíaca; ICFEp: insuficiência cardíaca com fração de ejeção preservada. 


\subsection{Tratamento da Insuficiência Cardíaca com Fração de Ejeção Preservada (ICFEp)}

Até o momento, ainda não há intervenção específica que reduza eventos cardiovasculares de pacientes com ICFEp. Os ensaios clínicos que avaliaram o uso de inibidores da enzima de conversão de angiotensina II (iECA), bloqueadores dos receptores de angiotensina II (BRA), inibidores da neprilisina e antagonistas dos receptores de angiotensina II (INRA) e espironolactona foram neutros quanto à redução do risco de eventos comparado ao placebo para pacientes com ICFEp. ${ }^{11-14}$ A análise de subgrupo, de acordo com a fração de ejeção, mostrou de maneira consistente a ausência de benefício nos subgrupos com fração de ejeção mais elevada (acima de 50\%). Achado semelhante foi encontrado para betabloqueadores em metanálise de ensaios clínicos randomizados. ${ }^{13}$ Por isso, as recomendações da diretriz de 2018 para o tratamento farmacológico da ICFEp continuam mantidas, incluindo o uso de diuréticos para aliviar congestão e o tratamento de comorbidades como a isquemia miocárdica, a fibrilação atrial e a hipertensão, para diminuir sintomas e potencialmente reduzir a progressão da ICFEp. ${ }^{15}$ Por isso, é fundamental que se investiguem condições potencialmente reversíveis e associadas à ICFEp "secundária", como as cardiomiopatias infiltrativas e restritivas, além de considerar causas alternativas de intolerância ao esforço.

\subsection{Tratamento da Insuficiência Cardíaca com Fração de Ejeção Levemente Reduzida (ICFEIr) (Tabela 1.4)}

Tabela 1.4 - Recomendações para o tratamento de insuficiência cardíaca com fração de ejeção levemente reduzida (ICFElr)

\begin{tabular}{|c|c|c|c|c|c|}
\hline Recomendações & Classe & NE & Comentários & $\begin{array}{c}\text { Tabela } \\
2018\end{array}$ & Ref. \\
\hline $\begin{array}{l}\text { Bisoprolol, carvedilol ou succinato de metoprolol para } \\
\text { pacientes com ICFEIr em ritmo sinusal para reduzir morbidade } \\
\text { e mortalidade. }\end{array}$ & Ila & A & \multirow{4}{*}{$\begin{array}{l}\text { NOVA: Os dados atualmente disponíveis } \\
\text { indicam que a resposta de pacientes com } \\
\text { ICFEIr ao tratamento da IC é semelhante à de } \\
\text { pacientes com ICFEr. }\end{array}$} & Nova & 13 \\
\hline iECA ou BRA para reduzir morbidade e mortalidade. & Ila & B & & Nova & 11 \\
\hline Espironolactona para reduzir morbidade e mortalidade & Ila & B & & Nova & 12 \\
\hline $\begin{array}{l}\text { Sacubitril-valsartana em substituição ao iECA (ou BRA), para } \\
\text { pacientes sintomáticos já em uso de terapêutica otimizada com } \\
\text { terapia tripla para reduzir hospitalização. }\end{array}$ & Ila & B & & Nova & 14 \\
\hline
\end{tabular}

Em que pese a inexistência de estudos que tenham avaliado intervenções terapêuticas dirigidas especificamente a pacientes com insuficiência cardíaca com fração de ejeção levemente reduzida (ICFEIr), as análises secundárias de ensaios clínicos em pacientes com ICFEr e ICFEp indicam que pacientes com ICFEIr (fração de ejeção ventricular esquerda, FEVE 41-49\%) podem se beneficiar das intervenções correntemente indicadas a pacientes com ICFEr (FEVE $\leq 40 \%$ ). Em metanálise de 11 estudos controlados e randomizados observou-se que os betabloqueadores se associaram a menor mortalidade em pacientes com ICFEi e ritmo sinusal. ${ }^{13} \mathrm{Em}$ subanálise do estudo Topcat, foi identificado o efeito benéfico da espironolactona na mortalidade cardiovascular de pacientes com FEVE entre 44-50\%; ${ }^{12}$ em subanálise do CHARM houve benefício com candesartan no desfecho combinado de mortalidade cardiovascular e hospitalizações nos pacientes com FEVE de $40 \%$ a $49 \% .{ }^{11}$ A análise combinada dos estudos PARAGON-HF e PARADIGM-HF sugeriu que sacubitrilvalsartana está associada à redução de hospitalizações em níveis intermediários de FEVE, sendo este efeito mais intenso entre os pacientes do sexo feminino com valores mais elevados de FEVE. ${ }^{14}$

Esta diretriz utiliza as denominações e definições de acordo com a nova classificaçao universal de IC. ICFEp: insuficiência cardiaca com fração de ejeção preservada; ICFElr: insuficiência cardíaca com fração de ejeção levemente reduzida; ICFEr: insuficiência cardíaca com fração de ejeção reduzida; FEVE: fração de ejeção ventricular esquerda; iECA: inibidores da enzima de conversão de angiotensina Il; BRA: bloqueadores dos receptores de angiotensina II. 
1.4. Tratamento da Insuficiência Cardíaca com fração de Ejeção Melhorada (ICFEm) (Tabela 1.5)

Tabela 1.5 - Recomendações para o tratamento de insuficiência cardíaca com fração de ejeção melhorada (ICFEm)

\begin{tabular}{|c|c|c|c|c|c|}
\hline Recomendações & Classe & NE & Comentários & $\begin{array}{c}\text { Tabela } \\
2018\end{array}$ & Ref. \\
\hline $\begin{array}{l}\text { Manutenção da terapêutica modificadora de prognóstico } \\
\text { utilizada no tratamento da ICFEr por cardiomiopatia } \\
\text { dilatada melhorada. }\end{array}$ & 1 & B & $\begin{array}{l}\text { NOVA: Indicação respaldada por estudo } \\
\text { randomizado multicêntrico com amostra limitada e } \\
\text { com desfechos substitutos. }\end{array}$ & Nova & 16 \\
\hline
\end{tabular}

0 avanço no tratamento da IC com fração de ejeção reduzida (ICFEr) tem determinado a melhora na FEVE e a redução no tamanho do ventrículo esquerdo em cerca de $40 \%$ dos pacientes, dependendo da etiologia. ${ }^{17}$ Recentemente, foi publicada a nova definição e classificação universal de IC, que recomenda o termo IC com fração de ejeção melhorada (ICFEm) para pacientes com FEVE prévia < $40 \%$ e tiveram um aumento de 10 pontos percentuais atingindo taxas acima de $40 \%$. Esta classificação universal recomenda que seja utilizado o termo "melhorada" ao invés de "recuperada". ${ }^{18}$ Halliday BP et al. ${ }^{16}$ testaram a segurança de retirar a medicação para IC em um grupo pequeno de pacientes com cardiomiopatia dilatada recuperada em um ensaio clínico piloto, sem cegamento, mas conduzido de modo randomizado e multicêntrico. Os critérios de inclusão foram: diagnóstico prévio de cardiomiopatia dilatada com FEVE menor ou igual a 40\%; ausência de sintomas de insuficiência cardíaca; tratamento com diurético de alça e medicações modificadoras de prognóstico; FEVE atual maior ou igual que 50\%; volume diastólico final do ventrículo esquerdo indexado à superfície corporal normal e NT-proBNP menor que $250 \mathrm{pg} / \mathrm{mL}$. Os pacientes foram randomizados para a retirada das medicações por 6 meses e o desfecho primário foi considerado uma combinação de redução da FEVE, dilatação do VE e retorno dos sintomas de IC. Após 6 meses de seguimento, 44\% dos pacientes que tiveram sua medicação retirada apresentaram algum dos critérios para desfecho primário, comparados a nenhum participante do grupo de tratamento mantido, registrando-se uma taxa de eventos estimada em 45,7\% (IC 95\% 28,5-67,2; p = 0,0001). Este estudo, apesar de pequeno e com desenho aquém do ideal, é a melhor evidência até o momento sobre essa população, sugerindo que a manutenção dos fármacos nesse contexto seja a melhor estratégia, pelo menos até a publicação de estudo mais robusto.

Esta diretriz utiliza as denominações e definições de acordo com a nova classificaçao universal de IC. ${ }^{18}$ FEVE: fração de ejeção ventricular esquerda; ICFEr: insuficiência cardíaca com fração de ejeção reduzida; NT-proBNP: fração N-terminal do peptídeo natriurético atrial do tipo B.

\section{Inovações em Amiloidose Cardíaca}

Assistimos recentemente a grandes avanços no conhecimento da amiloidose cardíaca, o que acarretou na profunda transformação do seu significado clínico, epidemiológico e no surgimento de tratamentos específicos. Várias evidências sugerem que a amiloidose cardíaca não seja uma doença rara, mas uma condição amplamente subdiagnosticada, considerada hoje uma causa relativamente comum e tratável de insuficiência cardíaca com fração de ejeção preservada (ICFEp), particularmente a amiloidose cardíaca ligada à transtirretina (ATTR) na sua forma selvagem ou sistêmica senil (ATTR-wt), cujo diagnóstico tem aumentado de forma expressiva. ${ }^{19-22}$

Trata-se de uma doença multisistêmica causada pela deposição tecidual de proteínas fibrilares insolúveis que perdem a sua conformação, o que leva à disfunção orgânica, inclusive do coração. Mais de 30 tipos de proteínas amiloidogênicas são descritas, ${ }^{23}$ sendo duas delas responsáveis por 95\% dos casos de acometimento cardíaco: a amiloidose por cadeia leve $(\mathrm{AL})$, esta relacionada com a produção monoclonal de imunoglobulinas devido à discrasia de plasmócitos; e amiloidose pela transtiretina (ATTR), a proteína transportadora de retinol e tiroxina produzida pelo fígado, que pode ter caráter secundário à sua mutação (ATTRm) ou ser selvagem (ATTRwt), causada por alterações pós-transcricionais e as proteínas de chaperonas, ligadas ao envelhecimento.
A AL apresenta incidência de 6 a 10 milhões de pessoas por ano e era considerada a principal causa de amiloidose cardíaca. ${ }^{24}$ No entanto, com o desenvolvimento de técnicas não invasivas de diagnóstico e o surgimento de tratamentos efetivos, o diagnóstico da ATTR, especialmente da ATTRwt, tem aumentado significativamente. ${ }^{19}$ Estudos demonstram ATTR em até $13 \%$ dos pacientes com ICFEp e espessamento da parede ventricular esquerda maior que $12 \mathrm{~mm},{ }^{20}$ sendo que até $25 \%$ das necropsias de muito idosos apresentam TTR no coração. ${ }^{22}$ A ATTRm apresenta um caráter autossômico dominante, com mais de 130 mutações descritas, que causam variações nos fenótipos de acometimento neurológico e cardíaco.

\subsection{Quando Suspeitar de Amiloidose}

Tendo em vista que a ATTR, particularmente a ATTRwt, é uma condição mais prevalente do que se antecipava, é importante suspeitar dessa condição na presença de pistas clínicas para posterior investigação diagnóstica (Tabela 2.1). Por se tratar de uma forma de cardiomiopatia restritiva infiltrativa, o padrão típico é espessamento da parede ventricular, da disfunção diastólica e dos distúrbios de condução. Em certos contextos clínicos, é necessário o diagnóstico diferencial com cardiomiopatia hipertrófica, ICFEp ${ }^{25}$ bloqueios atrioventriculares avançados e arritmias atriais sem causa aparente. A concomitância de ATTRwt e estenose aórtica cálcica pode causar hipertrofia ventricular acentuada e se apresentar como estenose aórtica de baixo fluxo e baixo gradiente. 
Além disso, certas manifestações multisistêmicas podem levantar suspeita de ATTR: síndrome de túnel do carpo bilateral, ruptura do tendão do bíceps, hipotensão ortostática, estenose do canal vertebral, alterações digestivas e intolerância a medicações anti-hipertensivas. ${ }^{26} \mathrm{~A}$ história familiar é muito importante nas formas hereditárias da amiloidose, que apresentam prognóstico pior do que nos pacientes acometidos com a forma selvagem da doença.

\subsection{Diagnóstico de Amiloidose Cardíaca (Tabela 2.1)}

Diante da suspeita da doença, o primeiro passo é investigar a presença de cadeias leves de imunoglobulinas para o diagnóstico da $\mathrm{AL}$, uma vez que essa forma da amiloidose cardíaca exige tratamento específico com quimioterápicos e o prognóstico piora muito com o retardo no início do tratamento. A confirmação da $\mathrm{AL}$ depende da detecção da proteína amiloide em tecidos envolvidos (biopsia), mas a forma ATTR pode ser confirmada não invasivamente, mediante emprego de cintilografia cardíaca com radiotraçadores ósseos. No Brasil, é usado o Tc-99m-pirofosfato.

Tabela 2.1 - Pistas clínicas para o diagnóstico de amiloidose

\section{História e Exame Físico}

ICFEp, particularmente em homens idosos (maiores de 65 anos)

Intolerância ao iECA/BRA/INRA e ou betabloqueadores

Síndrome do túnel do carpo bilateral

Estenose do canal vertebral

Ruptura do tendão do bíceps

Polineuropatia periférica não explicada e/ou disfunção autonômica (hipotensão postural; diarreia alternada com constipação; disfunção erétil)

Equimose periorbitária

Macroglossia

Pistas Originadas dos Exames de Imagem

Espessamento concêntrico das paredes do VE com amplitude do QRS reduzida ou não aumentada proporcionalmente ao grau de aumento da espessura das paredes do VE

Fenótipo infiltrativo ao ecocardiograma (SIV>12mm), hiperrefringência miocárdica, hipertrofia biventricular, derrame pericárdico, espessamento valvar, espessamento de septo interatrial

Redução do strain longitudinal que poupa a região apical (apical sparing)

Enchimento ventricular esquerdo de padrão restritivo, com espessamento das paredes do ventrículo direito

Realce tardio de contraste na ressonância magnética cardíaca de padrão subendocárdico ou transmural, difuso ou aumento do volume extracelular

Pistas Combinadas

Insuficiência cardíaca exibindo cavidade ventricular esquerda não dilatada e com espessamento das paredes (septo interventricular maior que 12 mm), especialmente em pacientes sem hipertensão arterial sistêmica pregressa.

Apresentação clínica de cardiomiopatia hipertrófica iniciada tardiamente (acima de 60 anos)

Estenose aórtica com espessamento das paredes do ventrículo direito, particularmente nos casos paradoxais com baixo fluxo/baixo gradiente

ICFEp: insuficiência cardíaca de fração com ejeção preservada; FEVE: fração de ejeção ventricular esquerda; iECA: inibidores da enzima de conversão de angiotensina II; BRA: bloqueadores dos receptores de angiotensina II; INRA: inibidores da neprilisina e antagonistas dos receptores de angiotensina II. 


\subsection{Métodos Diagnósticos}

\subsubsection{Eletrocardiograma}

A baixa voltagem no complexo QRS é achado comum na $\mathrm{AL}$, sendo menos prevalente na ATTR (aproximadamente $30 \%$ dos casos), sendo mais comum a discrepância entre a magnitude da hipertrofia ao ecocardiograma e a amplitude dos complexos QRS. Fibrilação atrial e o padrão de "pseudoinfarto" também podem ser encontrados.

\subsubsection{Ecocardiograma}

É um dos principais exames para levantar a suspeita. Entre os achados sugestivos se destacam o espessamento da parede ventricular esquerda maior que $12 \mathrm{~mm}$, especialmente na ausência de hipertensão arterial, aumento bi-atrial e desproporcional ao tamanho dos ventrículos, espessamento das valvas atrioventriculares e do septo interatrial, e o aumento da ecogenicidade do miocárdio com aparência granular. $\mathrm{O}$ índice de deformação sistólica longitudinal do miocárdio ou strain sistólico longitudinal pode mostrar a preservação da contratilidade do ápice do ventrículo esquerdo com relação aos demais segmentos (apical sparing ou imagem de "cereja de bolo"). ${ }^{27}$

\subsubsection{Cintilografia Cardíaca com Radiotraçadores} Ósseos

Cintilografia cardíaca com radiotraçadores ósseos, como Tc99m-pirofosfato usado no Brasil, pode ser utilizada para o diagnóstico diferencial entre a amiloidose AL e ATTR, esta última mostrando captação miocárdica anômala com intensidade maior ou equivalente à óssea. No entanto, a captação cardíaca pode ocorrer, ainda que mais discreta, em até $30 \%$ dos casos de AL. A captação cardíaca intensa (grau 2 ou 3), em conjunto com ausência de cadeias leves nos exames bioquímicos, tem especificidade de 100\% para ATTR, podendo dispensar a biopsia cardíaca para o diagnóstico da doença. ${ }^{19}$

\subsubsection{Ressonância Magnética Cardíaca}

A Ressonância Magnética Cardíaca possui alta sensibilidade e especificidade para o diagnóstico, sendo útil também para diferenciar a amiloidose cardíaca de outras miocardiopatias. A deposição amiloide no miocárdio causa aumento no volume de distribuição do contraste paramagnético nas regiões do miocárdio em que os cardiomiócitos são substituídos ou deslocados por fibrose ou inflamação, cursando com padrão de realce tardio (RT) mais comumente subendocárdico difuso e circunferencial do ventrículo esquerdo, ainda que realces tardios transmural e difuso também possam ser encontrados. ${ }^{27}$

\subsection{Tratamento de Amiloidose Cardíaca por Transtirretina (AC-ATTR) (Tabela 2.2)}

Frente à importância clínica e epidemiológica, além de novas terapias emergentes para esta doença, um Posicionamento sobre Diagnóstico e Tratamento da Amiloidose Cardíaca será publicado em breve, abordando de forma mais ampla os diferentes aspectos da doença.

Tabela 2.2 - Recomendações para o tratamento específico da amiloidose cardíaca por transtirretina (AC-ATTR)

\begin{tabular}{lccc}
\hline Recomendação & Classe & NE & Comentário \\
\hline $\begin{array}{l}\text { Tafamidis } 80 \text { mg/dia, para o tratamento de pacientes com } \\
\text { amiloidose cardíaca por transtirretina para redução da } \\
\text { mortalidade e de hospitalizações cardiovasculares. }\end{array}$ & I & B & $\begin{array}{c}\text { Tabela } \\
\mathbf{2 0 1 8}\end{array}$ \\
\hline
\end{tabular}

Várias etapas do processo de formação das fibrilas amiloides constituem alvos terapêuticos na amiloidose por transtirretina (ATTR). A primeira terapia modificadora da doença que demonstrou evidência de benefício em pacientes com cardiomiopatia amiloide é um estabilizador dos tetrâmeros da TTR, o tafamidis. Esse fármaco foi testado em um estudo clínico multicêntrico e randomizado contra o placebo, envolvendo 441 pacientes com AC e no qual 264 deles receberam o medicamento em doses de $20 \mathrm{mg}$ ou $80 \mathrm{mg}$ ao dia (estudo ATTR-ACT [Tafamidis Treatment for Patients with Transthyretin Amyloid Cardiomyopathy]). ${ }^{28}$ Os principais resultados mostraram que o uso de tafamidis se associou à redução de $30 \%$ no desfecho primário de mortalidade por qualquer causa ( $R R=0,70$ [IC 95\%: 0,51-0,96]), além de reduzir as internações por causa cardiovascular em 32\% (RR = 0,68 [IC95\%: 0,56 -0,81]) e a piora da capacidade funcional e da qualidade de vida. Esses resultados embasaram, no Brasil, a aprovação pela Anvisa do uso do fármaco para o tratamento da amiloidose cardíaca por TTR na dose de $80 \mathrm{mg} / \mathrm{dia}^{28}$

ATTR: amiloidose por transtirretina; $R$ R: risco relativo. 


\section{Atualização}

\section{Inovações em Telemonitoramento na Insuficiência Cardíaca (Tabela 3.1)}

Tabela 3.1 - Recomendações de telemonitoramento, werables, inteligência artificial e machine learning na insuficiência cardíaca

\begin{tabular}{|c|c|c|c|c|c|}
\hline Recomendações & Classe & NE & Comentário & $\begin{array}{c}\text { Tabela } \\
2018\end{array}$ & Ref. \\
\hline $\begin{array}{l}\text { Uso de monitoramento e suporte à distância } \\
\text { (telemonitoramento) para manejo de pacientes com } \\
\text { insuficiência cardíaca crônica. }\end{array}$ & Ila & A & $\begin{array}{l}\text { NOVA: Existem metanálises mostrando diminuição } \\
\text { na mortalidade e na internação por IC. }\end{array}$ & Nova & $29-32$ \\
\hline $\begin{array}{l}\text { Wearables como ferramentas auxiliares no manejo } \\
\text { diagnóstico, terapêutico e de reabilitação em pacientes } \\
\text { com insuficiência cardíaca crônica ou aguda. }\end{array}$ & Ila & B & $\begin{array}{l}\text { NOVA: Vários estudos observacionais mostram o } \\
\text { benefício do uso de wearables em paciente com IC. }\end{array}$ & Nova & 33,34 \\
\hline $\begin{array}{l}\text { Uso da Inteligência artificial no diagnóstico, avaliação de } \\
\text { prognóstico, ou seleção de pacientes com maior benefício } \\
\text { para diversas terapias. }\end{array}$ & Ilb & B & $\begin{array}{l}\text { NOVA: Estudos observacionais apontam para o } \\
\text { benefício do uso de Machine Learning e Inteligência } \\
\text { Artificial no diagnóstico e prognóstico da IC. }\end{array}$ & Nova & 35 \\
\hline
\end{tabular}

As metanálises envolvendo estudos observacionais e randomizados de monitoramento e suporte à distância invasivo ou não invasivo têm mostrado impacto positivo no prognóstico de pacientes com IC. ${ }^{29-32} \mathrm{~A}$ redução na mortalidade geral pode variar de 19 a $31 \%$ com o telemonitoramento em pacientes com IC enquanto a redução na frequência de internação hospitalar por IC varia de 27 a 39\%, principalmente em pacientes em classe funcional (CF) III/IV, segundo a New York Heart Association (NYHA). A inteligência artificial apresenta aplicações em estudo para IC, seja como diagnóstico, avaliação de prognóstico, telemonitoramento ou ainda para selecionar pacientes com maior benefício para diversas terapias. ${ }^{33,34}$ Isso pode ser feito, por exemplo, na distinção de fenótipos, alocando pacientes em diferentes perfis de assinatura de doença; na melhor acurácia para o diagnóstico de IC aguda com relação ao médico; e no eventual direcionamento para terapias novas ou já estabelecidas, como análise adicional do ECG basal para identificar paciente melhor respondedor à terapia de ressincronização cardíaca. ${ }^{35}$

CF: classe funcional; IC: insuficiência cardíaca.

\section{Inovações em Cardiointervenção}

4.1. Abordagem Percutânea da Insuficiência Mitral Secundária (Tabela 4.1)

Tabela 4.1 - Recomendações para intervenção percutânea na insuficiência mitral grave secundária

\begin{tabular}{|c|c|c|c|c|c|}
\hline Recomendação & Classe & NE & Comentários & $\begin{array}{l}\text { Tabela } \\
2018\end{array}$ & Ref. \\
\hline \multicolumn{6}{|l|}{ Clipagem percutânea da valva mitral } \\
\hline \multicolumn{6}{|l|}{ Isquêmica ou dilatada } \\
\hline $\begin{array}{l}\text { Sintomas refratários (classe funcional } \geq I I \text { ) ao tratamento } \\
\text { clínico convencional e após avaliação do Heart Team. }\end{array}$ & Ila & B & $\begin{array}{l}\text { NOVA: Estudo randomizado com desfecho de } \\
\text { morte respalda esta recomendação. }\end{array}$ & $\begin{array}{l}\text { Item } 11.3 \\
\text { (página 467) }\end{array}$ & 36 \\
\hline
\end{tabular}

Recomendamos que a terapia guiada por diretrizes esteja otimizada, incluindo terapia de ressincronização cardíaca e revascularização, quando apropriado, antes da consideração do tratamento percutâneo da insuficiência mitral (IM) para pacientes com ICFEr e IM grave. 0 estudo COAPT (Transcatheter Mitral-Valve Repair in Patients with Heart Failure) que avaliou se o uso do dispositivo edge-to-edge poderia beneficiar pacientes com IM secundária moderadamente grave ou grave (EROA maior ou igual a $30 \mathrm{~mm}^{2}$ e/ou volume regurgitante maior que $45 \mathrm{~mL}$ ) com FEVE de $20 \%$ a $50 \%$, diâmetro sistólico final do VE menor que $7 \mathrm{~cm}$ e sintomas persistentes, apesar da terapia baseada em evidências maximizada, com participação de equipe multidisciplinar experiente na avaliação e tratamento da IC e IM. ${ }^{36}$ A atualização da Diretriz de Valvopatia de 2020 não fez esta diferenciação na seleção dos pacientes. Visando manter a linearidade das diretrizes, optamos por também não incluir esta diferenciação em nossa recomendação. ${ }^{37}$

ICFEr: insuficiência cardiaca com fração de ejeção reduzida; IM: insuficiência mitral; VE: ventrículo esquerdo; IC: insuficiência cardiaca; FEVE: fração de ejeção ventricular esquerda. NYHA: New York Heart Association. 


\subsection{Ablação de Fibrilação Atrial (Tabela 4.2)}

Tabela 4.2 - Recomendações para ablação de fibrilação atrial na insuficiência Cardíaca com Fração de Ejeção reduzida (ICFEr)

\begin{tabular}{|c|c|c|c|c|c|}
\hline Recomendação & Classe & NE & Comentários & $\begin{array}{c}\text { Tabela } \\
2018\end{array}$ & Ref. \\
\hline $\begin{array}{l}\text { Ablação de FA para restaurar o ritmo sinusal em } \\
\text { pacientes sintomáticos, intolerantes ou refratários a } \\
\text { fármacos antiarrítmicos para redução de mortalidade } \\
\text { e hospitalizações por IC. }\end{array}$ & Ila & B & Recomendação de 2018 mantida. & $\begin{array}{c}\text { Item } 10.1 \\
\text { (página 465) }\end{array}$ & $\begin{array}{l}\text { Vide } \\
2018\end{array}$ \\
\hline $\begin{array}{l}\text { Ablação de FA como alternativa ao tratamento } \\
\text { clínico com fármacos antiarrítmicos para sintomas } \\
\text { refratários ou pacientes intolerantes. }\end{array}$ & I & A & $\begin{array}{l}\text { NOVA: Estudos randomizados demonstram maior } \\
\text { taxa de sucesso de manutenção do ritmo sinusal } \\
\text { com ablação da FA, além da ausência dos efeitos } \\
\text { colaterais causados pelos fármacos antiarrítmicos. }\end{array}$ & $\begin{array}{c}\text { Item } 10.1 \\
\text { (página 465) }\end{array}$ & $38-43$ \\
\hline $\begin{array}{l}\text { Ablação de FA para promover remodelamento } \\
\text { reverso em pacientes com taquicardiomiopatia } \\
\text { induzida pela FA, se refratários ao tratamento } \\
\text { medicamentoso ou na preferência do paciente pela } \\
\text { ablação, independentemente dos sintomas. }\end{array}$ & I & $\mathrm{B}$ & $\begin{array}{l}\text { NOVA: Estudo randomizado demonstrou a } \\
\text { capacidade da ablação da FA em promover } \\
\text { remodelamento reverso em pacientes com IC por } \\
\text { provável taquimiocardiopatia. }\end{array}$ & $\begin{array}{c}\text { Item } 10.1 \\
\text { (página 465) }\end{array}$ & 39,44 \\
\hline
\end{tabular}

A ablação de fibrilação atrial (FA) em pacientes com IC tem um benefício maior do que o uso de fármacos antiarrítmicos pela maior taxa de manutenção de ritmo sinusal, melhora de capacidade funcional e qualidade de vida, CF, distância no teste de caminhada de 6 minutos, VO ${ }_{2}$ máximo e redução de biomarcadores (BNP). Ela pode ser considerada uma terapia alternativa para controle de sintomas em pacientes com FA e que são intolerantes ou refratários ao tratamento com antiarrítmicos ou mesmo como terapia inicial. ${ }^{38-43}$ Remodelamento reverso foi observado em diversos estudos com ablação de FA, gerando incremento de FEVE. ${ }^{38-42,44}$ Quando a etiologia da IC é desconhecida e considera-se taquicardiomiopatia induzida pela FA como etiologia possível, o incremento esperado de FEVE com a ablação é ainda mais significativo. ${ }^{39,44}$ Estudos também demostraram redução de $45 \%$ de hospitalização por IC, $47-56 \%$ mortalidade por qualquer causa e de $38 \%$ de morte ou hospitalização por IC.41,42,44 Entretanto, a taxa de sucesso varia em torno de 60 a $80 \%$ no primeiro ano e a doença cardíaca estrutural é um fator de risco para recorrência. ${ }^{45} \mathrm{O}$ isolamento das veias pulmonares pode ser feito por radiofrequência ou crioablação e essas técnicas podem ser combinadas com ablação de outros substratos.

CF: classe funcional; FA: fibrilação atrial; IC: insuficiência cardiaca. BNP: peptídeo natriurético do tipo B; FEVE: fração de ejeção ventricular esquerda.

\section{COVID-19 e a Insuficiência Cardíaca (Tabela 5.1)}

Tabela 5.1 - Recomendações relacionadas com o manejo da COVID-19 em pacientes com insuficiência cardíaca

\begin{tabular}{lcccc}
\hline Recomendação & Classe & NE & Comentários \\
\hline $\begin{array}{l}\text { Testagem com RT-PCR para SARS-CoV-2 em indivíduos } \\
\text { com IC crônica e manifestações respiratórias agudas. }\end{array}$ & I & C & $\begin{array}{c}\text { NOVA: Editoriais e recomendações de sociedades } \\
\text { (publicação on-line). }\end{array}$ \\
\hline $\begin{array}{l}\text { Manutenção de iECA, BRA ou INRA em indivíduos com } \\
\text { IC crônica que desenvolvam COVID-19, na ausência de } \\
\text { hipotensão ou sinais de comprometimento hemodinâmico. }\end{array}$ & I & C & $\begin{array}{c}\text { NoVA: Estudos observacionais controlados com } \\
\text { número grande de pacientes, mas com percentual } \\
\text { menor de pacientes com IC. }\end{array}$ \\
\hline $\begin{array}{l}\text { Acompanhamento ambulatorial de pacientes com IC por } \\
\text { meio de visitas virtuais e telemonitoramento durante } 0 \\
\text { período de pandemia por COVID-19. }\end{array}$ & I & C & NoVA: Recomendações de experts e sociedades. & Nova \\
\hline
\end{tabular}

Considerando que sintomas de COVID-19 podem mimetizar descompensação de IC, a testagem para SARS-CoV-2 com RT-PCR é recomendável tanto no cenário de pacientes atendidos em sala de emergência como em casos ambulatoriais. ${ }^{46,47}$ Não há evidências que contraindiquem a manutenção de iECA, BRA ou INRA em pacientes com IC que apresentem quadro de COVID-19. Durante o período infeccioso, a manutenção ou não destas medicações deve ser guiada pelo quadro clínico e/ou hemodinâmico, individualmente. ${ }^{48-50}$ Ferramentas virtuais e/ou remotas (telefonemas, telemonitoramento, consultas on-line, videochamadas, entre outras) podem ser utilizadas para manter o cuidado de pacientes com IC durante a pandemia por COVID-19. Estas medidas, que reduzem a circulação de pessoas e a exposição dos pacientes ao vírus, têm-se mostrado eficientes no cuidado e tendem a consolidar-se no período pós-pandêmico. No caso de pacientes em período de maior instabilidade clínica (pós-alta de episódio de descompensação ou IC de início recente) e de candidatos a terapias avançadas para IC (transplante ou dispositivos de assistência ventricular), é recomendável manter ao menos uma visita presencial em intervalos variáveis e intercalados com visitas virtuais, especialmente considerando que durante a pandemia há uma tendência à redução do número de transplantes realizados, prorrogando o tempo de espera em fila. ${ }^{51,52}$

IC: insuficiência cardiaca; iECA: inibidores da enzima de conversão de angiotensina II; BRA: bloqueadores dos receptores de angiotensina II; INRA:

inibidores de neprilisina e antagonistas dos receptores de angiotensina II. 


\section{Inovações em Insuficiência Cardíaca Avançada}

\subsection{Definição de Insuficiência Cardíaca Avançada}

A história natural da IC caracteriza-se pela piora progressiva da função cardíaca e dos sintomas de IC. Apesar dos avanços no tratamento farmacológico e do impacto prognóstico dos dispositivos implantáveis como terapia de ressincronização cardíaca, pacientes com insuficiência cardíaca podem progredir para uma condição clínica denominada IC avançada, em que o tratamento tradicional não é efetivo e as terapias avançadas, tais como transplante cardíaco, suporte com dispositivo de assistência circulatória mecânica (DACM) ou cuidados paliativos são necessárias.

Embora o termo IC avançada já venha sendo usado desde 2007, atualizações recentes foram descritas visando incluir situações clínicas que também podem requerer terapias avançadas e que não foram contempladas na primeira classificação, como pacientes com ICFEp com quadro restritivo grave e não limitando apenas aos pacientes com IC com fração de ejeção gravemente reduzida. ${ }^{1-3,4}$ Neste cenário, a disfunção ventricular direita grave isolada e as doenças valvares graves inoperáveis, bem como anormalidades congênitas também podem ser consideradas causas de disfunção cardíaca grave (Tabela 6.1). ${ }^{53-68}$

Estes critérios variam de acordo com as diferentes sociedades de cardiologia, porém a presença de sintomas graves persistentes; a capacidade reduzida ao exercício; e episódios recorrentes de congestão pulmonar ou sistêmica que necessitem hospitalizações estão presentes em todas elas como descrito na Tabela 6.2.

É importante ressaltar que o reconhecimento precoce de um paciente com IC avançada é fator determinante para o seu prognóstico, uma vez que isto permitirá o encaminhamento para um centro especializado que disponha das terapias avançadas necessárias para a condução do caso.

Uma técnica mnemônica particularmente útil e que pode ajudar a identificar pacientes que precisam de encaminhamento a um especialista em IC é o I-NEED-HELP, que integra a história clínica, as hospitalizações e a intolerância medicamentosa, além de sintomas e a disfunção de órgãoalvo. (Tabela 6.3)

Tabela 6.1 - Critérios para a definição de insuficiência cardíaca avançada

\section{Critérios para a definição de IC avançada}

1. Sintomas de IC persistentes e graves (NYHA III ou IV).

2. Disfunção ventricular grave definida por:

- FEVE $\leq 30 \%$ ou

- IC direita isolada ou

- Alterações valvares graves não operáveis ou

- Anormalidades congênitas

Valores de BNP ou NT-proBNP persistentemente elevados e dados mostrando disfunção diastólica grave ou anormalidades estruturais do VE, de acordo com critérios de definição de ICFEp ou ICFElr.

3. Episódios de congestão pulmonar ou sistêmica que requerem altas doses de diurético endovenoso (ou combinação de diuréticos) ou episódios de baixo débito que requerem uso de inotrópicos ou fármacos vasoativos ou arritmias malignas que causem mais que uma visita não planejada à emergência ou hospitalização nos últimos 12 meses.

4. Capacidade para o exercício gravemente reduzida, com inabilidade para o exercício ou baixa capacidade no teste de caminhada de 6 minutos (TC6min $<300 \mathrm{~m})$ ou $\mathrm{VO}_{2}$ pico $\left(<12-14 \mathrm{~mL} \cdot \mathrm{kg}^{-1} \cdot \mathrm{min}^{-1}\right)$, estimado de origem cardíaca.

Adaptado de Metra M et al. ${ }^{65}$ Eur J Heart Fail. 2007; 9(6-7): 684-94; Metra M et al. ${ }^{66}$ Cardiac Fail Rev. 2019; Crespo-Leiro MG et al. ${ }^{67}$ Eur J Heart Fail. 2018; 20(11): 505-35; Trusby LK et al., ${ }^{68}$ JACC Heart Fail. 2020; 8(7): 523-36.

IC: insuficiência cardiaca; NYHA: New York Heart Association; FEVE: fração de ejeção ventricular esquerda; BNP: peptídeo natriurético do tipo B; NTproBNP: fração N-terminal do peptídeo natriurético do tipo B; ICFEp: insuficiência cardíaca com fração de ejeção preservada; ICFElr: insuficiência cardiaca com fração de ejeção levemente reduzida; TC6m: teste de caminhada de 6 minutos; VE: ventrículo esquerdo; VO ${ }_{2}$ consumo de oxigênio. 
Tabela 6.2 - Critérios propostos por diferentes sociedades de cardiologia para identificação de pacientes com IC avançada

\begin{tabular}{|c|c|c|c|c|}
\hline Critério & SBC & ACC/AHA & ESC & HFSA \\
\hline Sintomas graves e persistentes apesar de terapia otimizada & $\checkmark$ & $\checkmark$ & $\checkmark$ & $\checkmark$ \\
\hline Limitação funcional importante (classe funcional NYHA III ou IV) & $\checkmark$ & $\checkmark$ & $\checkmark$ & $\checkmark$ \\
\hline Dispneia persistente com atividades da vida diária & & $\checkmark$ & & \\
\hline Hospitalizações recorrentes & $\checkmark$ & $\checkmark$ & $\checkmark$ & $\checkmark$ \\
\hline Visitas frequentes não planejadas à emergência & $\checkmark$ & & $\checkmark$ & $\checkmark$ \\
\hline Intolerância a otimização terapêutica máxima & $\checkmark$ & $\checkmark$ & & $\checkmark$ \\
\hline Lesão em órgão-alvo & $\checkmark$ & $\checkmark$ & & $\checkmark$ \\
\hline Hiponatremia persistente & $\checkmark$ & $\checkmark$ & & $\checkmark$ \\
\hline Congestão pulmonar ou sistêmica refratária a diureticoterapia & $\checkmark$ & $\checkmark$ & & $\checkmark$ \\
\hline Choques de cardiodesfibrilador implantável frequentes & $\checkmark$ & $\checkmark$ & & $\checkmark$ \\
\hline Caquexia cardíaca & $\checkmark$ & $\checkmark$ & & $\checkmark$ \\
\hline Pressão arterial sistólica frequentemente $\leq 90 \mathrm{mmHg}$ & & $\checkmark$ & & \\
\hline Valores de BNP ou NT-proBNP persistentemente elevados & $\checkmark$ & & $\checkmark$ & \\
\hline Disfunção grave de VE com fração de ejeção reduzida (FEVE < 30\%) & $\checkmark$ & & $\checkmark$ & $\checkmark$ \\
\hline Disfunção grave de VE com padrão ecocardiográfico pseudonormal ou restritivo & $\checkmark$ & & $\checkmark$ & \\
\hline Pressões de enchimento elevadas (PCP $>16 \mathrm{mmHg}+/-\mathrm{PVC}>12 \mathrm{mmHg}$ ) & & & $\checkmark$ & \\
\hline Baixa capacidade no TC6m $(<300 \mathrm{~m})$ ou VO ${ }_{2}$ pico $<12-14 \mathrm{~mL} \cdot \mathrm{kg}^{-1} \cdot \mathrm{min}^{-1}$ & $\checkmark$ & & $\checkmark$ & $\checkmark$ \\
\hline Dependência de inotrópicos intravenosos & $\checkmark$ & & & $\checkmark$ \\
\hline Disfunção progressiva de VD e HP secundária & $\checkmark$ & & & $\checkmark$ \\
\hline
\end{tabular}

Adaptado de Metra M et al. ${ }^{65}$ Eur J Heart Fail. 2007; 9(6-7): 684-94; Metra M et al. ${ }^{66}$ Cardiac Fail Rev 2019; Crespo-Leiro MG et al. ${ }^{67}$ Eur J Heart Fail. 2018; 20(11): 505-35; Trusby LK et al.68 JACC Heart Fail. 2020; 8(7): 523-36.

ACC/AHA: American College of Cardiology/American Heart Association CDI: cardiodesfibrilador implantável; IC: insuficiência cardiaca; BNP: peptídeo natriurético do tipo B; ESC: European Society of Cardiology; HFSA: Heart Failure Society of America; HP: hipertensão pulmonar; NYHA: New York Heart Association; NT-proBNP: fração N-terminal do peptídeo natriurético do tipo B; PCP: pressão capilar pulmonar; PVC: pressão venosa central; TC6m: teste de caminhada de 6 minutos; VD: ventrículo direito; VE: ventrículo esquerdo; $V O_{2}$ : consumo de oxigênio.

Tabela 6.3 - Sinais de alerta no paciente com IC avançada

\begin{tabular}{ll}
\hline $\mathrm{I}$ & Inotrópico dependente/intolerância à terapia otimizada \\
\hline $\mathrm{N}$ & NYHA III/IV persistente \\
\hline $\mathrm{E}$ & Ejeção (fração) menor que 20\% \\
\hline $\mathrm{E}$ & Edema persistente, refratário a doses progressivas de diuréticos \\
\hline $\mathrm{D}$ & Desfibrilador (choque apropriado recorrente) \\
\hline $\mathrm{H}$ & Hospitalizações e visitas à emergência recorrentes nos últimos 12 meses \\
\hline $\mathrm{E}$ & Elevação persistente de peptídeos natriuréticos \\
\hline $\mathrm{L}$ & Lesão em órgão-alvo \\
\hline $\mathrm{P}$ & Pressão arterial sistólica persistentemente menor que $90 \mathrm{mmHg}$ \\
\hline
\end{tabular}




\section{Atualização}

\subsection{Papel do Especialista em Insuficiência Cardíaca Avançada}

Com a delimitação cada vez mais clara de um perfil específico de pacientes enquadrados na definição mais contemporânea de IC avançada, cresce também a necessidade de definir a importância do papel do especialista em IC avançada nos centros especializados. Este profissional deverá ser familiarizado (e treinado) para atender potenciais cadidatos ao transplante cardíaco (e posterior seguimento) e pacientes em choque cardiogênico (CC). Ele assume a liderança na coordenação dos trabalhos envolvendo o time de choque e, portanto, está familiarizado com as diversas (e crescentes) opções de uso de assistência circulatória. Por fim, este profissional deve ser capaz de compreender o momento e as implicações de discutir cuidados paliativos e diretivas antecipadas de vontade para pacientes não elegíveis para transplante cardíaco, bem como o uso de dispositivos de longa permanência.

\subsection{Abordagem do Paciente com Insuficiência Cardíaca} Avançada (Figura 6.1)

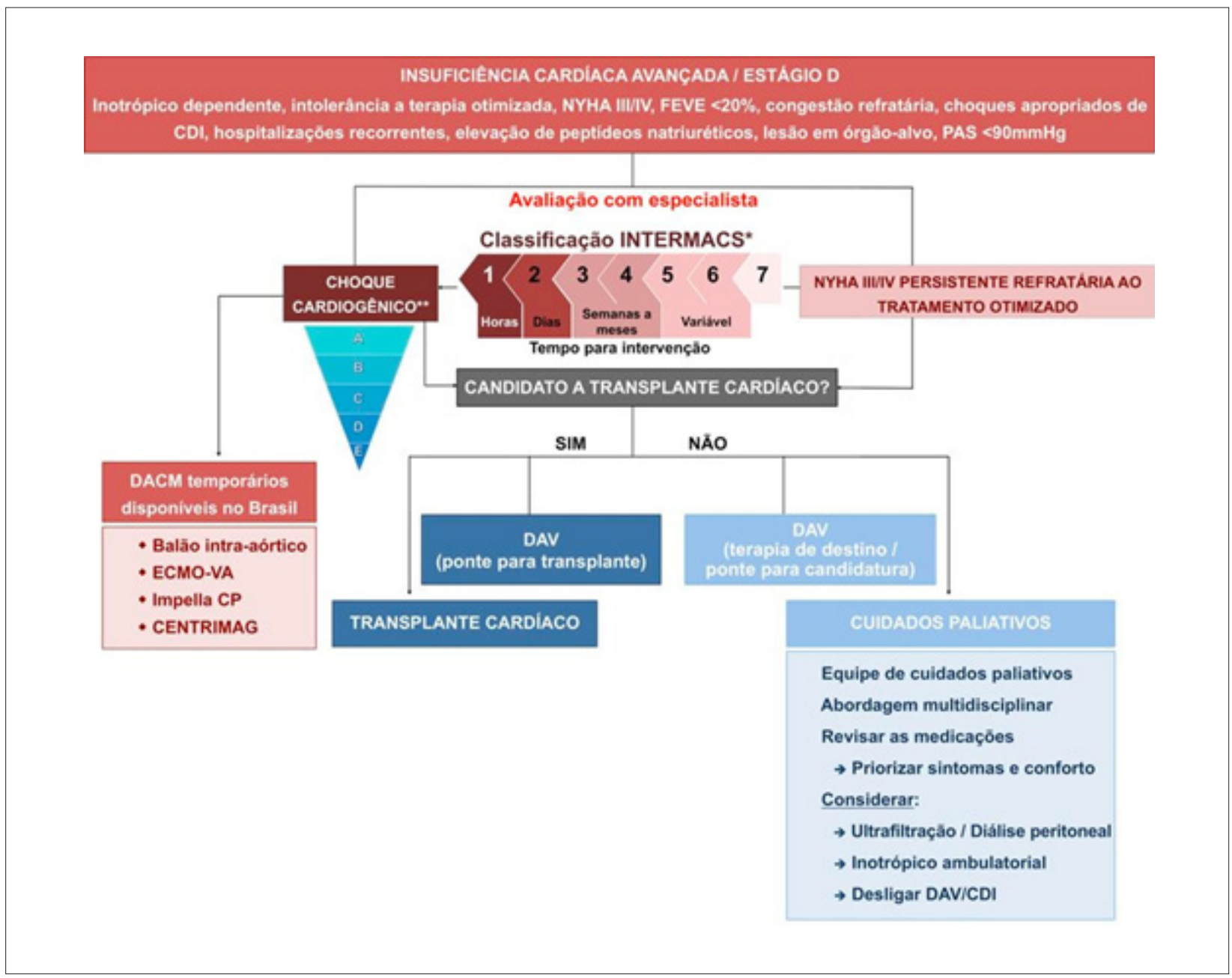

Figura 6.1 - Algoritmo de tratamento do paciente com insuficiência cardíaca avançada.

*Classificação clínica de pacientes com insuficiência cardíaca avançada da Interagency Registry for Mechanically Assisted Circulatory Support (Intermacs), vide Diretriz Brasileira de Insuficiência Cardíaca Crônica e Aguda. ${ }^{15}$ Arq Bras Cardiol. 2018; Quadro 4.6 (página 505).

${ }^{*}$ Classificação de choque cardiogênico proposta pela Society for Cardiovascular Angiography and Interventions (SCAl). Estágio A: sob risco de choque; Estágio B: início do choque; Estágio C: choque clássico; Estágio D: choque em deterioração; Estágio E: choque extremo. Adaptado de Baran DA et al. ${ }^{58}$ Catheter Cardiovasc Interv. 2019; 94(1): 29-37.

CDI: cardiodesfibrilador implantável; CF: classe funcional; DACM: dispositivos de assistência circulatória mecânica; DAV: dispositivo de assistência ventricular implantável; ECMO-VA: circuito de oxigenação por membrana extracorpórea venoarterial; IC: insuficiência cardiaca; NYHA: New York Heart Association. 
6.4. Inovações sobre o Manejo da Congestão em Pacientes com Insuficiência Cardíaca Avançada (Tabela 6.4)

Tabela 6.4 - Monitorização ambulatorial da congestão na insuficiência cardíaca

\begin{tabular}{lccc}
\hline Orientações & Classe & NE & Comentário \\
\hline $\begin{array}{l}\text { Monitorização invasiva remota da congestão, através de dispositivo } \\
\text { implantável na artéria pulmonar para reduzir hospitalizações e } \\
\text { mortalidade em pacientes com ICFEr ambulatoriais. }\end{array}$ & Ila & $\begin{array}{l}\text { NOVA: A orientação atual reflete dados } \\
\text { de estudos randomizados pequenos e } \\
\text { estudos de vida real, com impacto em } \\
\text { redução de hospitalizações e mortalidade. }\end{array}$ & $\begin{array}{c}\text { Nova } \\
\text { 30,53-57 }\end{array}$ \\
\hline
\end{tabular}

Embora poucas inovações tenham sido observadas nos últimos anos com relação ao manejo da congestão na IC avançada, algumas evidências surgiram com relação à monitorização da congestão, o que impactou no prognóstico de pacientes com IC. Neste campo, existem dados recentes sobre a monitorização não invasiva através de telemonitoramento, mostrando impacto em redução de dias perdidos com admissão hospitalar e morte por todas as causas. ${ }^{30}$ Também foram encontrados dados sobre monitorização invasiva através de dispositivo implantável na artéria pulmonar, CardioMEMs, que permite a transmissão de medidas de pressão arterial pulmonar (PAP) para uma central e, por conseguinte, ao médico do paciente. 0 impacto desta monitorização invasiva foi testado no estudo CHAMPION (CardioMEMS Heart Sensor Allows Monitoring of Pressure to Improve Outcomes in NYHA Class III Heart Failure Patients trial), que envolveu pacientes ambulatoriais com IC (NYHA III) e mostrou redução de $28 \%$ nas hospitalizações por IC. Nos pacientes que receberam ao menos duas medicações da terapia padrão para IC, a monitorização invasiva associou-se a $57 \%$ de redução de mortalidade..$^{53}$ Esta estratégia mostrou-se eficaz e segura em estudos de "vida real", ${ }^{54}$ além de custo-efetiva. ${ }^{55}$ Estes dados foram confirmados recentemente em estudo conduzido por centros na Europa ${ }^{56} \mathrm{e}$ em estudo prospectivo multicêntrico aberto que envolveu 1.200 pacientes em CF III, que demonstrou redução significativa de hospitalização por IC com baixas taxas de complicações relacionadas com o implante do sensor no seguimento de um ano. ${ }^{57}$ Trata-se de uma estratégia promissora com potencial a ser acrescentada à prática clínica.

CF: classe funcional; IC: insuficiência cardiaca; NYHA: New York Heart Association; PAP: pressão de artéria pulmonar. 


\section{Atualização}

\subsection{Classificação Atual de Choque Cardiogênico}

Em 2019, a Society for Cardiovascular Angiography and Interventions (SCAI) propôs uma nova classificação para o CC, visando facilitar a identificação das diferentes fases de deterioração clínica e a necessidade de intensificação do tratamento. ${ }^{58,59}$ As cinco fases dessa classificação incorporam achados de hipoperfusão tecidual e os sinais de disfunção orgânica que permitem uma definição hemodinâmica simples e maior discriminação entre os perfis INTERMACS (Interagency Registry for Mechanically Assisted Circulatory Support) (Figura 6.2, Tabela 6.5).

O estágio A inclui pacientes sob risco de choque cardiogênico, enquanto os estágios B a E descrevem fases progressivas do choque cardiogênico convencional. A diferença entre os estágios B e C é a presença de hipoperfusão, que está presente nos estágios $C$ e superiores. $\mathrm{O}$ estágio $\mathrm{D}$ indica que as medidas de manejo inicial do choque cardiogênico não foram suficientes para restaurar a estabilidade hemodinâmica ou a perfusão tecidual após pelo menos 30 minutos de observação, enquanto o estágio $\mathrm{E}$ caracteriza casos extremos, no qual os pacientes se apresentam hemodinamicamente instáveis e frequentemente em colapso circulatório. Pacientes em estágios SCAI D e E apresentam maior mortalidade e podem se beneficiar da transferência precoce para centros especializados, capazes de oferecer modalidades avançadas de suporte circulatório. ${ }^{59}$

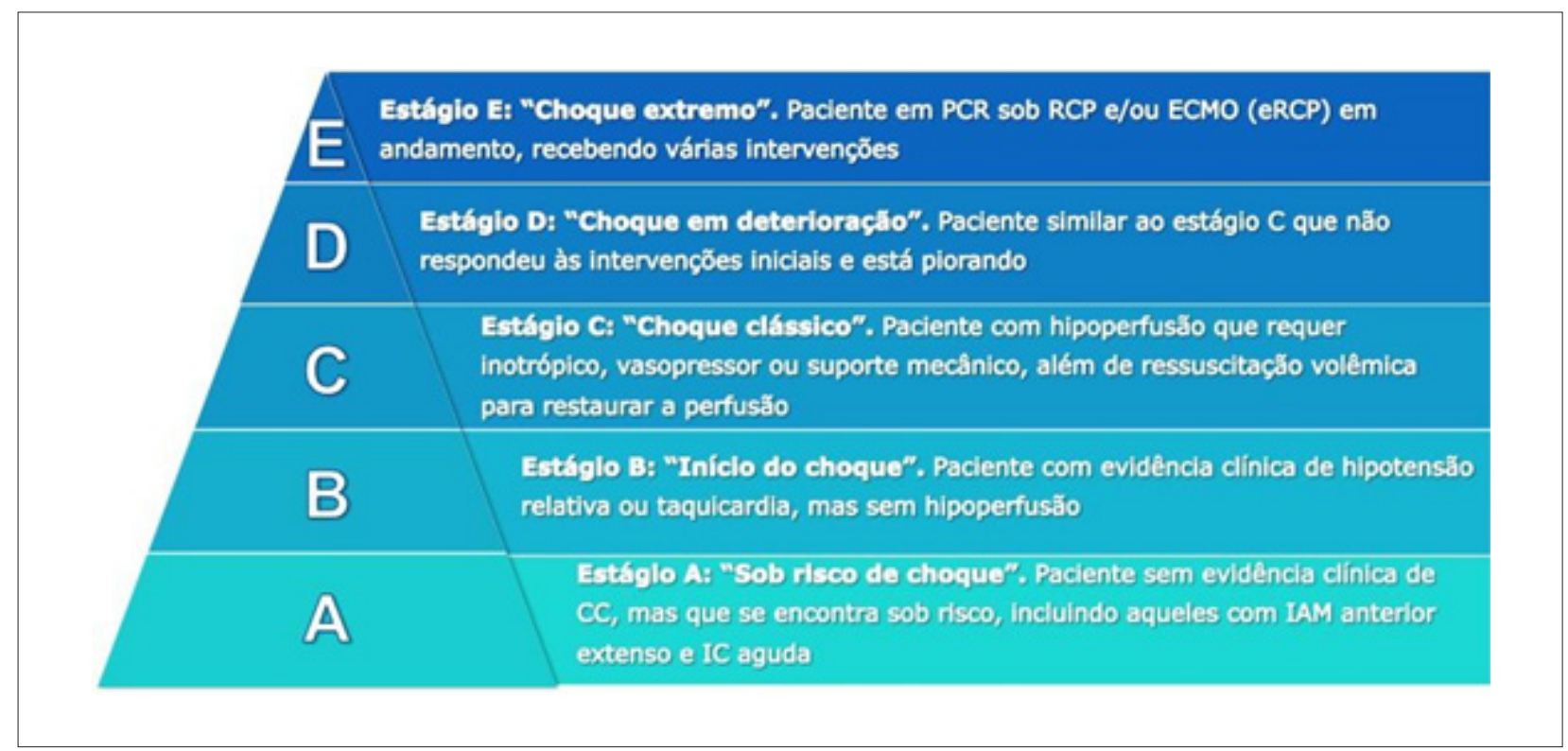

Figura 6.2 - Classificação da Society for Cardiovascular Angiography and Interventions (SCAl) para o choque cardiogênico. Adaptado de: Baran DA et al. ${ }^{58}$ SCAl clinical expert consensus statement on the classification of cardiogenic shock. Catheter Cardiovasc Interv. 2019; 94(1): 29-37.

CC: choque cardiogênico; ECMO: oxigenação por membrana extracorporal; IAM: infarto agudo do miocárdio; IC: insuficiência cardíaca; PCR: parada cardiorrespiratória; RCP: ressuscitação cardiorrespiratória. 
Tabela 6.5 - Descritores das fases do choque cardiogênico: exame físico, biomarcadores e achados hemodinâmicos

\begin{tabular}{|c|c|c|c|}
\hline Estágio do CC & Achados clínicos & Biomarcadores & Hemodinâmica \\
\hline A (sob risco) & $\begin{array}{l}\text { PVJ normal } \\
\text { Ausculta pulmonar limpa } \\
\text { Perfil quente e seco } \\
\text { Pulsos periféricos cheios } \\
\text { Estado mental preservado }\end{array}$ & $\begin{array}{l}\text { Bioquímica normal } \\
\text { Função renal normal } \\
\text { Lactato normal }\end{array}$ & $\begin{array}{rl}\mathrm{PAS} \geq 100 & \mathrm{mmHg} \text { (ou normal para } \\
& \text { o paciente) } \\
& \text { Se CAP: } \\
\bullet & \mathrm{Cl} \geq 2,5 \mathrm{~L} / \mathrm{min} / \mathrm{m}^{2} \\
\cdot & \mathrm{PVC}<10 \mathrm{mmHg} \\
& \cdot \mathrm{SvO}_{2} \geq 65 \%\end{array}$ \\
\hline B (início) & $\begin{array}{l}\text { PVJ elevada } \\
\text { Estertores pulmonares } \\
\text { Perfil quente e seco } \\
\text { Pulsos periféricos cheios } \\
\text { Estado mental preservado }\end{array}$ & $\begin{array}{l}\text { Lactato normal } \\
\text { Disfunção renal mínima } \\
\text { BNP elevado }\end{array}$ & $\begin{array}{c}\text { PAS }<90 \text { OU PAM }<60 \text { OU queda }> \\
30 \mathrm{mmHg} \text { do basal } \\
\mathrm{FC} \geq 100 \mathrm{bpm} \\
\mathrm{Se} \mathrm{CAP:} \\
\mathrm{CI} \geq 2,2 \mathrm{~L} / \mathrm{min} / \mathrm{m}^{2} \\
\mathrm{SvO}_{2} \geq 65 \%\end{array}$ \\
\hline C (clássico) & $\begin{array}{c}\text { Pode incluir qualquer um dos } \\
\text { seguintes: } \\
\text { Mal-estar geral } \\
\text { Em pânico } \\
\text { Palidez e lividez } \\
\text { Sobrecarga de volume } \\
\text { Estertores pulmonares difusos } \\
\text { Classificação de Killip } 3 \text { ou } 4 \\
\text { BiPap ou ventilação mecânica } \\
\text { Extremidades frias e pegajosas } \\
\text { Alteração aguda do estado mental } \\
\text { Débito urinário < } 30 \mathrm{~mL} / \mathrm{h}\end{array}$ & $\begin{array}{c}\text { Pode incluir qualquer um dos } \\
\text { seguintes: } \\
\text { Lactato } \geq 2 \mathrm{mmol} / \mathrm{L} \\
\text { Creatinina dobrando ou queda }>50 \% \\
\text { na TFG } \\
\text { Provas hepáticas alteradas } \\
\text { BNP elevado }\end{array}$ & $\begin{array}{l}\text { Pode incluir qualquer um dos seguintes: } \\
\text { PAS }<90 \text { OU PAM }<60 \text { OU queda } \\
>30 \mathrm{mmHg} \text { do basal. Vasopressores e/ou } \\
\text { dispositivos para manter a PA } \\
\text { CAP: } \\
\qquad \mathrm{Cl}<2,2 \mathrm{~L} / \mathrm{min} / \mathrm{m}^{2} \\
\text { - } \mathrm{PCP}>15 \mathrm{mmHg} \\
\text { - PVC/PCP } \geq 0,8 \\
\text { - } \mathrm{PAPi}<1,85 \\
\bullet \mathrm{CPO} \leq 0,6 \mathrm{~W}\end{array}$ \\
\hline D (deterioração) & $\begin{array}{l}\text { Pode incluir qualquer um dos } \\
\text { achados do estágio } C\end{array}$ & $\begin{array}{c}\text { Pode incluir qualquer um dos } \\
\text { achados do estágio } C \text {, em } \\
\text { deterioração }\end{array}$ & $\begin{array}{l}\text { Pode incluir qualquer um dos achados } \\
\text { do estágio C e: } \\
\text { Múltiplos vasopressores e/ou dispositivos } \\
\text { de assistência circulatória mecânica para } \\
\text { manter perfusão }\end{array}$ \\
\hline E (extremo) & $\begin{array}{l}\text { Pulsos pouco palpáveis } \\
\text { Colapso circulatório } \\
\text { Sob ventilação mecânica } \\
\text { Desfibrilador em uso }\end{array}$ & $\begin{array}{c}\text { PCR (modificador }-\mathrm{A}^{*} \text { ) } \\
\mathrm{pH} \leq 7,2 \\
\text { Lactato } \geq 5 \mathrm{mmol} / \mathrm{L}\end{array}$ & $\begin{array}{c}\text { PAS inaudível / PCR } \\
\text { TVSP ou TV/FV refratária } \\
\text { Hipotensão apesar do suporte máximo }\end{array}$ \\
\hline
\end{tabular}

*O modificador $(A)$ é aplicado para descrever pacientes que tiveram uma parada cardíaca independentemente da duração.

Adaptado de Baran DA et al. ${ }^{58}$ SCAI clinical expert consensus statement on the classification of cardiogenic shock. Catheter Cardiovasc Interv. 2019; 94(1):29-37.

BiPap: ventilação por dois niveis de pressão positiva; BNP: peptideo natriurético do tipo-B; CAP: cateter de artéria pulmonar; CC: choque cardiogênico; IC: índice cardíaco; CPO: poder cardíaco, do inglês cardiac power output; FC: frequência cardiaca; FV: fibrilação ventricular; IAM: infarto agudo do miocárdio; IC: insuficiência cardíaca; PAM: pressão arterial média; PAPi: índice de pulsatilidade da artéria pulmonar; PAS: pressão arterial sistólica; PCP: pressão de capilar pulmonar; PVC: pressão venosa central; PVJ: pulso venoso jugular; SvO $0_{2}$ saturação venosa mista de oxigênio; TFG: taxa de filtração glomerular; TV: taquicardia ventricular; TVSP: taquicardia ventricular sem pulso. 


\title{
Atualização
}

\author{
6.6. Aplicabilidade do Cateter de Artéria Pulmonar na
}

Insuficiência Cardíaca Avançada (Tabela 6.6)

Tabela 6.6 - Recomendações de uso de cateter de artéria pulmonar em pacientes com insuficiência cardíaca avançada

\begin{tabular}{|c|c|c|c|c|c|}
\hline Recomendações & Classe & NE & Comentário & $\begin{array}{l}\text { Tabela } \\
2018\end{array}$ & Ref. \\
\hline $\begin{array}{l}\text { Em pacientes com IC avançada, candidatos a transplante } \\
\text { cardíaco ou suporte circulatório mecânico. }\end{array}$ & 1 & B & Recomendação de 2018 mantida. & $\begin{array}{l}\text { Item 2.2.6. } \\
\text { (página 495) }\end{array}$ & $\begin{array}{l}\text { Vide } \\
2018\end{array}$ \\
\hline $\begin{array}{l}\text { Para auxiliar no tratamento e suporte hemodinâmico de } \\
\text { pacientes com IC refratária ao tratamento padrão ou em } \\
\text { pacientes com choque cardiogênico. }\end{array}$ & Ila & B & $\begin{array}{l}\text { MODIFICADO: Novas evidências respaldam } \\
\text { a mudança de classe de recomendação. }\end{array}$ & $\begin{array}{l}\text { Item 2.2.6. } \\
\text { (página 495) }\end{array}$ & $\begin{array}{c}60- \\
61\end{array}$ \\
\hline
\end{tabular}

0 uso do cateter de artéria pulmonar (CAP) na monitorização hemodinâmica de pacientes hospitalizados com IC refratária permanece controverso. ${ }^{62,63}$ Em 2005, o estudo ESCAPE (Evaluation Study of Congestive Heart Failure and Pulmonary Artery Catheterization Effectiveness) não mostrou benefício do uso rotineiro do CAP no manejo de pacientes com IC descompensada sem CC. ${ }^{64}$ Entretanto, avanços recentes no campo dos dispositivos de assistência circulatória mecânica (DACM) têm favorecido a criação de algoritmos para o manejo do CC guiado por parâmetros do CAP. 0 reconhecimento precoce, a identificação do subtipo de choque e a compreensão do impacto esperado de cada tipo de dispositivo sobre parâmetros hemodinâmicos como débito cardíaco, pressão capilar pulmonar (PCP), pressão venosa central (PVC) e pressão arterial média (PAM) permite a escolha do DACM mais adequado para cada estágio do CC (Figura 6.1). Além disso, as informações obtidas através do CAP auxiliam na caracterização fenotípica do CC em choque predominantemente esquerdo (CPO $<0,6 \mathrm{~W}, \mathrm{PAPi}>1, \mathrm{PVC}<15 \mathrm{mmHg}$ e PCP $>15 \mathrm{mmHg}$ ), direito (CPO $<0,6 \mathrm{~W}, \mathrm{PAPi}<1, \mathrm{PVC}>15 \mathrm{mmHg}$ e PCP $<15$ $\mathrm{mmHg}$ ) ou biventricular (CPO $<0,6 \mathrm{~W}, \mathrm{PAPi}<1, \mathrm{PVC}>15 \mathrm{mmHg}$ e PCP $>15 \mathrm{mmHg}$ ). ${ }^{60,65-68}$ Recentemente, em um dos primeiros estudos do Cardiogenic Shock Working Group (CSWG), Garan et al. ${ }^{61}$ avaliaram a associação entre o manejo do CC guiado por parâmetros do CAP e a mortalidade hospitalar em 1.414 pacientes com CC, a maioria com indicação de DACM e em estágio D da classificação SCAI. 0 manejo do CC guiado por parâmetros do CAP obtidos antes do implante de DACM foi associado a redução significativa de mortalidade, principalmente nos estágios mais avançados de CC (estágios D ou E da classificação SCAI). ${ }^{61}$ É importante enfatizar que o CAP é uma ferramenta diagnóstica, não terapêutica, e sua efetividade dependerá de decisões clínicas tomadas pelo time de profissionais envolvidos com o manejo do CC.

CAP: cateter de artéria pulmonar; CC: choque cardiogênico; CPO: poder cardíaco, do inglês cardiac power output; DACM: dispositivos de assistência circulatória mecânica; IC: insuficiência cardiaca; PAM: pressão arterial média; PAP: índice de pulsatilidade da artéria pulmonar; PCP: pressão de capilar pulmonar; PVC: pressão venosa central.

6.7. Inovações com Relação aos Dispositivos de Assistência Circulatória de Curta Duração na Insuficiência Cardíaca Avançada (Tabela 6.7)

Tabela 6.7 - Recomendações para descompressão do ventrículo esquerdo em pacientes com oxigenação por membrana extracorpórea (ECM0)

\begin{tabular}{lccccc}
\hline Recomendações & Classe & NE & Comentário & $\begin{array}{c}\text { Tabela } \\
\mathbf{2 0 1 8}\end{array}$ & $\begin{array}{l}\text { Ref. } \\
\text { Considerar estratégias para descompressão do ventrículo esquerdo } \\
\text { em pacientes com suporte de assistência circulatória mecânica por }\end{array}$ \\
$\begin{array}{l}\text { ECMO venoarterial periférica e evidência de distensão ventricular } \\
\text { associada à hipocontratilidade acentuada e à congestão pulmonar. }\end{array}$ & lla & C & $\begin{array}{l}\text { NOVA: A recomendação atual reflete dados } \\
\text { de estudos observacionais e metanálises. }\end{array}$ & Nova & 69-73 \\
\hline
\end{tabular}

0 uso de oxigenação por membrana extracorpórea (ECMO) venoarterial periférica é caracterizado pelo aumento na pós-carga do VE causado pelo fluxo sanguíneo da cânula de retorno arterial, que pode agravar a hipocontratilidade cardíaca gerando distensão ventricular e congestão pulmonar. Em muitos casos, a redução no fluxo da ECMO combinada à terapia inotrópica pode ser suficiente para descomprimir o VE. ${ }^{74}$ No entanto, em casos refratários, outros métodos de descompressão podem ser usados, incluindo septostomia atrial; implante cirúrgico de cateter transapical; descompressão percutânea da artéria pulmonar pela veia jugular; e dispositivo de assistência circulatório mecânica (DACM) como balão intra-aórtico (BIA), Impella ${ }^{\circledR}$, ou CentriMag ${ }^{\circledR}$. Em estudos observacionais, a descompressão do VE se associou à redução de mortalidade, à maior recuperação miocárdica e ao menor tempo de desmame da ECMO em pacientes com CC tratados com ECMO venoarterial periférica. ${ }^{69-72}$ Cada técnica de descompressão apresenta riscos inerentes que devem ser considerados individualmente de acordo com a etiologia da doença de base, as limitações do sítio de acesso, a presença de coagulopatias, a disponibilidade dos DACMs e a experiência de cada centro ${ }^{75}$ Apesar das limitações conhecidas, o BIA permanece o dispositivo mais utilizado, com uma metanálise recente sugerindo menor risco de complicações como acidente vascular cerebral, isquemia periférica e hemólise, da descompressão por BIA em comparação com outros métodos, às custas do aumento de sangramento. ${ }^{73}$ No entanto, nenhum ensaio clínico randomizado foi realizado até o momento para estabelecer 0 método ideal de descompressão do VE, e estudos prospectivos são necessários. Também não há consenso se a descompressão do VE deva ser realizada preventivamente ou como medida de resgate. Algumas indicações reconhecidas para descompressão do VE são: pressão capilar pulmonar (PCP) elevada, VE distendido e hipocontrátil ou com evidência ecocardiográfica de estase sanguínea, diminuição da abertura valva aórtica durante 0 ciclo cardíaco, hipoxemia, edema pulmonar progressivo e arritmia ventricular refratária.

CC: choque cardiogênico; DACM: dispositivo de assistência circulatório mecânica; ECMO: oxigenação por membrana extracorpórea; PCP: pressão

capilar pulmonar; VE: ventrículo esquerdo. 


\author{
6.8. Inovações com Relação aos Cuidados Paliativos na \\ Insuficiência Cardíaca Avançada (Tabela 6.8)
}

Tabela 6.8 - Uso de inotrópicos intravenosos em regime ambulatorial para pacientes com insuficiência cardíaca avançada, não elegíveis para transplante cardíaco ou dispositivos de assistência circulatória mecânica

\begin{tabular}{lccc}
\hline Recomendações & Classe & NE & $\begin{array}{c}\text { Tabela } \\
\mathbf{2 0 1 8}\end{array}$ \\
\hline $\begin{array}{l}\text { Terapia inotrópica ambulatorial intravenosa contínua como tratamento } \\
\text { paliativo para o controle de sintomas em pacientes com IC avançada, } \\
\text { que não são elegíveis para dispositivos de assistência circulatória } \\
\text { mecânica ou transplante cardíaco. }\end{array}$ & Ilb & C & $\begin{array}{c}\text { NoVA: A recomendação atual reflete } \\
\text { dados de estudos com limitações de } \\
\text { desenho execução. }\end{array}$ \\
\hline $\begin{array}{l}\text { Uso intermitente de inotrópico ou inodilatador para melhora dos } \\
\text { sintomas em pacientes com IC avançada ou para paliação em } \\
\text { pacientes sem outras opções de terapias avançadas. }\end{array}$ & Ilb & B & $\begin{array}{c}\text { NovA: Novas evidências de ECR e } \\
\text { metanálise de moderada qualidade } \\
\text { respaldam a recomendação. }\end{array}$ \\
\hline
\end{tabular}

A qualidade da evidência avaliando riscos e benefícios da terapia paliativa com inotrópico intravenoso em regime ambulatorial para pacientes com IC avançada é limitada e composta principalmente por estudos observacionais e sem um grupo controle. Metanálises de pequenos estudos randomizados controlados e estudos observacionais heterogêneos sugerem um potencial benefício clínico da terapia inotrópica ambulatorial contínua ou intermitente em pacientes com IC avançada, que não são elegíveis para DACM ou transplante cardíaco. ${ }^{76-78}$ Dentre os benefícios, destacam-se 0 alívio sintomático e a redução nas taxas de readmissão hospitalar. Entretanto, a necessidade de um cateter central para infusão contínua de inotrópicos está associada a maiores cuidados especiais e ao risco de infecções. 0 ensaio piloto LION-HEART (Efficacy and safety of intermittent intravenous outpatient administration of levosimendan in patients with advanced heart failure) randomizou 69 pacientes com IC avançada para receber placebo ou levosimendana intermitente, na dose de $0,2 \mu \mathrm{g} / \mathrm{kg} / \mathrm{min}$ por 6 horas, a cada 2 semanas e em 12 semanas demonstrou benefício do inotrópico com relação à redução de concentrações plasmáticas de NT-proBNP, escores de qualidade de vida e readmissões hospitalares, sem diferença nas taxas de eventos adversos entre os grupos. ${ }^{79}$ Até o momento, não há estudos de custoefetividade avaliando o impacto da infusão ambulatorial de inotrópicos como terapia paliativa para pacientes com IC avançada.

ECR: estudo controlado randomizado; IC: insuficiência cardiaca; NT: ProBNP-fração N-terminal do peptídeo natriurético do tipo B. 


\section{Tratamento da Insuficiência cardíaca com fração de ejeção reduzida (ICFEr)}

\author{
7.1. Estratégias Farmacológicas Previamente \\ Consolidadas para Tratamento da Insuficiência Cardíaca \\ com fração de Ejeção Reduzida (ICFEr) (Tabela 7.1)
}

Tabela 7.1 - Recomendações para tratamento farmacológico da insuficiência cardíaca com fração de ejeção reduzida (ICFEr) previamente consolidadas em 2018

\begin{tabular}{|c|c|c|c|c|c|}
\hline Recomendações & Classe & NE & Comentário & $\begin{array}{c}\text { Tabela } \\
2018\end{array}$ & Ref. \\
\hline $\begin{array}{l}\text { Bisoprolol, carvedilol e succinato de metoprolol para disfunção } \\
\text { de VE sintomática para reduzir morbidade e mortalidade. }\end{array}$ & 1 & A & Recomendação de 2018 mantida. & $\begin{array}{c}\text { Item } 7.2 \\
\text { (página 457) }\end{array}$ & $\begin{array}{l}\text { Vide } \\
2018\end{array}$ \\
\hline $\begin{array}{l}\text { iECA para disfunção de VE sintomática para reduzir morbidade } \\
\text { e mortalidade. }\end{array}$ & 1 & $A$ & Recomendação de 2018 mantida. & $\begin{array}{c}\text { Item } 7.1 \\
\text { (página 456) }\end{array}$ & $\begin{array}{l}\text { Vide } \\
2018\end{array}$ \\
\hline $\begin{array}{l}\text { BRA para disfunção de VE sintomática (nos intolerantes a iECA } \\
\text { por tosse/angioedema) para reduzir morbidade e mortalidade. }\end{array}$ & 1 & A & Recomendação de 2018 mantida. & $\begin{array}{c}\text { Item } 7.1 \\
\text { (página 456) }\end{array}$ & $\begin{array}{l}\text { Vide } \\
2018\end{array}$ \\
\hline $\begin{array}{l}\text { Antagonista dos receptores mineralocorticoides para disfunção } \\
\text { de VE sintomática, associada ao tratamento padrão com iECA/ } \\
\text { BRA/INRA e BB, para reduzir morbidade e mortalidade. }\end{array}$ & 1 & $A$ & $\begin{array}{l}\text { MODIFICADO: } 0 \text { uso de antagonista dos } \\
\text { receptores mineralocorticoides justifica-se } \\
\text { em pacientes em uso iECA/BRA ou INRA. }\end{array}$ & $\begin{array}{c}\text { Item } 7.3 \\
\text { (página 457) }\end{array}$ & $\begin{array}{l}80- \\
84\end{array}$ \\
\hline $\begin{array}{l}\text { Sacubitril-valsartana, em substituição ao iECA (ou BRA), para } \\
\text { disfunção de VE sintomática, já em uso de terapêutica otimizada } \\
\text { e com terapia tripla para reduzir morbidade e mortalidade. }\end{array}$ & 1 & B & Recomendação de 2018 mantida. & $\begin{array}{l}\text { Item } 7.4 \\
\text { (página } 458)\end{array}$ & $\begin{array}{l}\text { Vide } \\
2018\end{array}$ \\
\hline $\begin{array}{l}\text { Associação de hidralazina e nitrato para disfunção } \\
\text { sistólica sintomática em classe funcional II-IV (NYHA) } \\
\text { com contraindicação à iECA/BRA (insuficiência renal e/ou } \\
\text { hipercalemia) independentemente de raça ou para pacientes } \\
\text { negros autodeclarados com disfunção sistólica sintomática em } \\
\text { NYHA III-IV, apesar de terapêutica otimizada. }\end{array}$ & 1 & $\mathrm{~B}$ & Recomendação de 2018 mantida. & $\begin{array}{c}\text { Item } 7.7 \\
\text { (página 459) }\end{array}$ & $\begin{array}{l}\text { Vide } \\
2018\end{array}$ \\
\hline $\begin{array}{l}\text { Ivabradina para disfunção de VE sintomática, em paciente com } \\
\text { terapêutica otimizada, em ritmo sinusal e com FC maior que } 70 \\
\text { bpm para redução de hospitalização, morte cardiovascular e } \\
\text { morte por IC. }\end{array}$ & $\| A$ & B & Recomendação de 2018 mantida. & $\begin{array}{l}\text { Item } 7.5 \\
\text { (página } 458)\end{array}$ & $\begin{array}{l}\text { Vide } \\
2018\end{array}$ \\
\hline $\begin{array}{l}\text { Digoxina para disfunção de VE sintomática, apesar de } \\
\text { terapêutica otimizada com terapia tripla, para reduzir sintomas e } \\
\text { hospitalizações. }\end{array}$ & $\| A$ & B & Recomendação de 2018 mantida. & $\begin{array}{c}\text { Item } 7.6 \\
\text { (página } 458 \text { ) }\end{array}$ & $\begin{array}{l}\text { Vide } \\
2018\end{array}$ \\
\hline Diurético de alça para controle de congestão. & 1 & C & Recomendação de 2018 mantida. & $\begin{array}{c}\text { Item } 7.7 \\
\text { (página 459) }\end{array}$ & $\begin{array}{l}\text { Vide } \\
2018\end{array}$ \\
\hline $\begin{array}{l}\text { Diurético tiazídico, associado ao diurético de alça para } \\
\text { congestão persistente. }\end{array}$ & 1 & $\mathrm{C}$ & Recomendação de 2018 mantida. & $\begin{array}{c}\text { Item } 7.7 \\
\text { (página 459) }\end{array}$ & $\begin{array}{l}\text { Vide } \\
2018\end{array}$ \\
\hline
\end{tabular}

Nas últimas décadas, os avanços no tratamento farmacológico e no uso de dispositivos implantáveis trouxeram mudanças no prognóstico de pacientes com ICFEr ${ }^{80-91}$ No entanto, ainda existe risco residual e alta morbimortalidade mesmo após tratamento clínico otimizado já ter sido instituido. Tratamento clínico otimizado adicional. Nesta nova era, medicações que agem em diferentes mecanismos fisiopatológicos da IC surgem para complementar a ação exercida sobre o sistema neuro-humoral. É importante ressaltar que os benefícios observados com as novas medicações ocorreram em adição à terapia padrão otimizada, o que reforça a necessidade de manutenção da terapia tripla, que inclui betabloqueadores, bloqueadores do sistema renina-angiotensinaaldosterona (SRAA) e antagonistas mineralocorticoides. Uma vez instituída a terapia tripla e adicionadas as novas terapias que demonstraram benefício em redução de mortalidade cardiovascular, morte geral e hospitalização por IC, podemos também associar medicações que tiveram impacto em morbidade. A escolha destas terapias adicionais deve levar em consideração o perfil de cada paciente.

iECA: inibidores da enzima de conversão de angiotensina II; BRA: bloqueadores dos receptores de angiotensina II; INRA: inibidores da neprilisina e antagonista do receptor de angiotensina II; VE: ventrículo esquerdo; FC: frequência cardiaca; ICFEr: insuficiência cardíaca com fração de ejeção reduzida; IC: insuficiência cardíaca; SRAA: sistema renina-angiotensina-aldosterona. 


\subsection{Sacubitril-Valsartana (Tabela 7.2)}

Tabela 7.2 - Recomendações para o uso de sacubitril-valsartana em pacientes com insuficiência cardíaca com fração de ejeção reduzida (ICFEr)

\begin{tabular}{|c|c|c|c|c|c|}
\hline Recomendações & Classe & NE & Comentário & $\begin{array}{l}\text { Tabela } \\
2018\end{array}$ & Ref. \\
\hline $\begin{array}{l}\text { Sacubitril-valsartana, em substituição } \\
\text { ao iECA/BRA para disfunção de VE } \\
\text { sintomática, já em uso de terapêutica } \\
\text { otimizada com terapia tripla para reduzir } \\
\text { morbidade e mortalidade. }\end{array}$ & 1 & B & $\begin{array}{l}\text { Recomendação de } 2018 \\
\text { mantida. }\end{array}$ & $\begin{array}{c}\text { Item } 7.4 \\
\text { (página 458) }\end{array}$ & Vide 2018 \\
\hline $\begin{array}{l}\text { Sacubitril-valsartana, como início de } \\
\text { tratamento na IC crônica sintomática, pode } \\
\text { ser considerado no lugar de iECA ou BRA. }\end{array}$ & Ila & C & $\begin{array}{l}\text { NOVA: Análise de subgrupos } \\
\text { de estudos randomizado e } \\
\text { não randomizado mostram a } \\
\text { segurança de uso em pacientes } \\
\text { virgens de uso de iECA/BRA. }\end{array}$ & Nova & $84,92,93$ \\
\hline $\begin{array}{l}\text { Sacubitril-valsartana, em lugar de iECA/ } \\
\text { BRA, pode ser considerado em pacientes } \\
\text { hospitalizados com IC descompensada. }\end{array}$ & Ila & B & $\begin{array}{l}\text { NOVA: Estudo randomizado } \\
\text { usando desfecho substituto } \\
\text { (redução de biomarcadores) } \\
\text { respalda esta nova } \\
\text { recomendação. }\end{array}$ & Nova & $84,92,94$ \\
\hline
\end{tabular}

0 estudo PARADIGM-HF (Prospective Comparison of ARNi with ACE-I to Determine Impact on Global Mortality and Morbidity in Heart Failure) investigou, em pacientes com ICFEr, os efeitos sobre morbidade e mortalidade da atenuação da ação deletéria da Angiotensina II associada à potencialização do efeito protetor dos peptídeos natriuréticos endógenos pela inibição da neprilisina (enzima que degrada o BNP), utilizando uma nova classe de fármacos, os inibidores da neprilisina e dos receptores da Angiotensina II (INRAs), cuja molécula atualmente disponível é o sacubitril-valsartana, em comparação com enalapril. ${ }^{83}$ Foram investigados 8.442 pacientes com ICFEr sintomática ambulatorial com terapia clínica otimizada e que persistiam com FEVE $\leq 40 \%$, níveis elevados de peptídeos natriuréticos plasmáticos e clearance de creatinina estimado $\geq 30 \mathrm{~mL} / \mathrm{min} / 1,73 \mathrm{~m}^{2}$. Nesta população, sacubitril-valsartana foi superior ao enalapril, associado à redução de $21 \%$ das internações por piora da IC, de $20 \%$ na mortalidade cardiovascular, de $20 \%$ na morte súbita e $16 \%$ na mortalidade geral. Com base nos resultados do PARADIGM-HF, recomenda-se a troca deilECA/BRA para o sacubitril-valsartana nos pacientes com ICFEr que persistem sintomáticos, mesmo após o emprego de doses otimizadas dos bloqueadores neuro-hormonais. Mais recentemente, o estudo PIONEER-HF (Angiotensin-Neprilysin Inhibition in Acute Decompensated Heart Failure) comparou sacubitril-valsartana $(n=440)$ com enalapril $(n=441)$ em pacientes internados por IC descompensada, tendo como desfecho primário o tempo médio de mudança proporcional na concentração de NT-proBNP desde o início até a $4^{a}$ e a $8^{a}$ semanas. ${ }^{84}$ Os resultados mostraram uma redução significativa de NT-proBNP em maior grau com sacubitril-valsartana do que com enalapril, sendo esta redução observada já na primeira semana de tratamento e independentemente da história prévia de IC ou do uso prévio de iECA ou BRA. ${ }^{94}$ Os efeitos colaterais, hipercalemia, disfunção renal e hipotensão foram semelhantes em ambos os grupos. Em uma análise aberta, após 0 término das 8 semanas (Pioneer-HF extended) em que todos os pacientes receberam o sacubitril-valsartana por adicionais 4 semanas, observou-se uma queda adicional significante do NT-proBNP no grupo enalapril que passou a usar sacubitril-valsartana. ${ }^{92}$ Outro estudo prospectivo, observacional, o estudo TRANSITION (Initiation of sacubitril/valsartan in haemodynamically stabilised heart failure patients in hospital or early after discharge), ${ }^{93}$ iniciou sacubitril-valsartana em 1.002 pacientes durante a hospitalização por IC descompensada ou logo após a alta hospitalar e mostrou ser seguro e com boa tolerância, com metade dos pacientes atingindo a dose-alvo em 10 semanas, com poucos eventos adversos.$^{93} \mathrm{~A}$ partir dos resultados desses estudos, que sugerem ser seguro o uso de sacubitril-valsartana em pacientes internados por IC aguda descompensada após estabilização clínica, e a partir da extrapolação dos benefícios demonstrados no estudo PARADIGM-HF, o sacubitril- valsartana pode ser considerado, em lugar de iECA/BRA, para tratamento de pacientes hospitalizados com IC descompensada. Os resultados desses estudos recentes também indicam a segurança e tolerabilidade do início de tratamento com sacubitrilvalsartana, ao invés de iECAs/BRAs, em pacientes com ICFEr nova, que compuseram 34\% da casuística do estudo Pioneer-HF e $29 \%$ dos pacientes no estudo TRANSITION. ${ }^{84,92,93} \mathrm{Em}$ conjunto, estes dados sugerem que o início de sabubitril/valsartana para pacientes sem tratamento prévio com iECA/BRA e durante episódios de descompensação de IC seja aceitável e seguro. Dados a respeito deste tipo de intervenção em longo prazo e com desfechos, como mortalidade, ainda não estão disponíveis.

iECA: inibidores da enzima de conversão de angiotensina II; BRA: bloqueadores dos receptores de angiotensina II; INRA: inibidores da neprilisina e antagonista do receptor de angiotensina II; IC: insuficiência cardíaca; FEVE: fração de ejeção ventricular esquerda; VE: ventrículo esquerdo; FC: frequência cardíaca; ICFEr: insuficiência cardíaca com fração de ejeção reduzida; SRAA: sistema renina-angiotensina-aldosterona. 


\subsection{Inibidores de SGLT2 (Tabela 7.3)}

Tabela 7.3 - Recomendações para o uso de Inibidores de SGLT2 no tratamento de pacientes com Insuficiência cardíaca com fração de ejeção reduzida (ICFEr)

\begin{tabular}{|c|c|c|c|c|c|}
\hline Recomendações & Classe & NE & Comentário & $\begin{array}{c}\text { Tabela } \\
2018\end{array}$ & Ref. \\
\hline $\begin{array}{l}\text { Inibidores de SGLT2 (dapagliflozina ou empagliflozina) em pacientes } \\
\text { com ICFEr sintomáticos diabéticos ou não já com dose máxima } \\
\text { otimizada tolerada de betabloqueador, antagonista da aldosterona, } \\
\text { iECA/BRA ou INRA para reduzir desfechos cardiovasculares e } \\
\text { progressão da disfunção renal. }\end{array}$ & I & A & $\begin{array}{c}\text { NOVA: Os iSGLT2 são úteis para } \\
\text { redução de morte cardiovascular, e } \\
\text { hospitalização por insuficiência cardíaca. }\end{array}$ & Nova & $95-98$ \\
\hline
\end{tabular}

No DAPA-HF (Dapagliflozin and Prevention of Adverse Outcomes in Heart Failure), 4.744 pacientes com ICFEr foram randomizados para receber dapagliflozina ou placebo além da terapia-padrão, sendo $41,8 \%$ com DM2.$^{95} 0$ desfecho primário de morte cardiovascular ou agravamento da IC foi significativamente menor no grupo dapagliflozina ( $26 \%$ de redução). Quando analisados separadamente, houve redução significativa tanto na morte cardiovascular (18\% de redução) quanto na piora da IC (30\% de redução), independentemente de DM2. Tais resultados revelam uma nova terapia para IC, já aprovada para essa finalidade. O EMPEROR-Reduced (Empagliflozin Outcome Trial in Patients with Chronic Heart Failure and a Reduced Ejection Fraction) avaliou a empagliflozina versus placebo, além da terapia padrão, em 3.730 pacientes com ICFEr, sendo 50,2\% com DM2. ${ }^{96}$ Os pacientes apresentavam IC mais grave do que aqueles no Dapa-HF, com média de FEVE de $27 \%$ contra $31 \%$, sendo que mais de $70 \%$ dos pacientes tinham FEVE menor que $30 \%$, além de maior nível mediano de NT-proBNP (1.907 versus 1.437 pg/mL). Houve redução de $25 \%$ no desfecho primário de morte cardiovascular ou hospitalização por insuficiência cardíaca (HIC) em favor da empagliflozina. Quando analisados separadamente, não houve redução de morte cardiovascular, resultado diferente daquele observado no Dapa-HF. Novamente, o benefício foi visto independentemente da presença de DM2. Esses dados confirmam os resultados do DAPA-HF e reforçam a justificativa para o uso de inibidores do cotransportador de sódio e glicose 2 (iSGLT2) em pacientes com ICFEr para redução dos sintomas, melhora da qualidade de vida, redução do risco de hospitalização e morte cardiovascular.

A metanálise utilizando os resultados do DAPA-HF e do EMPEROR-Reduced, envolvendo 8.474 pacientes, mostrou redução de $13 \%$ na morte por todas as causas (HR combinado 0,87, IC de 95\% 0,77-0,98; $p=0,018)$ e redução de $14 \%$ nas mortes por doenças cardiovasculares $(0,86$, IC de $95 \%$ 0,76 - 0,98; $p$ = 0,027).(94) 0 uso dos iSGLT2 foi acompanhado por uma redução relativa de $26 \%$ no risco combinado de morte cardiovascular ou primeira hospitalização por IC $(0,74,0,68-0,82$; p < 0,0001), e por uma redução de $25 \%$ no composto de hospitalizações recorrentes por IC ou morte cardiovascular $(0,75$, $0,68-0,84 ; p<0,0001) .0$ risco do desfecho renal composto também foi reduzido $(0,62,0,43-0,90 ; p=0,013)$. A subanálise do DAPA-HF avaliou a eficácia e segurança da dapagliflozina em pacientes com ICFEr, de acordo com a taxa de filtração glomerular (TFG) basal, bem como os efeitos da dapagliflozina na TFG após a randomização. 0 efeito da dapagliflozina nos desfechos primários (morte CV ou piora da IC) e secundários não foi alterado pela TFG (<60 e $\geq 60 \mathrm{~mL} / \mathrm{min} / 1,73 \mathrm{~m}^{2}$ ). Desfecho composto renal pré-especificado (redução sustentada $>50 \%$ da TFG, doença renal terminal ou morte renal) também foi analisado, juntamente com a piora da TFG ao longo do estudo. Embora dapagliflozina não tenha reduzido o desfecho renal composto (RR = 0,71, IC 95\% $0,44-1,16, p=0,17)$, a taxa de piora da TFG foi menor com a dapagliflozina $(-1,09)$ versus placebo $(-2,87)$, $p<0,001$, em pacientes com ou sem DM2 ( $p$ de interação $=0,92) \cdot{ }^{95}$ No estudo EMPEROR-Reduced, a taxa anual de redução da TFG foi menor com a empagliflozina do que com o placebo (-0,55 versus $-2,28 \mathrm{~mL} / \mathrm{min} / 1,73 \mathrm{~m}^{2}$ por ano, $\left.\mathrm{p}<0,001\right)$ e os pacientes tratados com empagliflozina tiveram menor risco de desfechos renais sérios, independentemente da presença ou ausência de DM2. ${ }^{96}$ Os dados da subanálise do DAPA-HF e do EMPEROR-Reduced sugerem que o uso dos inibidores de SGLT2 é seguro em pacientes com ICFEr e pacientes com alteração da TFG, independentemente da presença de DM2.

BRA: bloqueadores dos receptores de angiotensina II; DM2: diabetes tipo 2; FEVE: fração de ejeção ventricular esquerda; IC: insuficiência cardíaca; ICFEr: insuficiência cardíaca com fração de ejeção reduzida; iECA: inibidores da enzima de conversão de angiotensina II; INRA: inibidores da neprilisina e antagonista do receptor de angiotensina II; iSGLT2: inibidores do cotransportador de sódio e glicose 2; TFG: taxa de filtração glomerular. 
7.4. Tratamento de Comorbidades na Insuficiência Cardíaca com fração de Ejeção Reduzida

7.4.1. Diabetes Tipo 2 (Tabela 7.4)

Tabela 7.4 - Recomendações para o uso de inibidores de SGLT2 na prevenção de hospitalização por insuficiência cardíaca em pacientes diabéticos tipo 2

\begin{tabular}{|c|c|c|c|c|c|}
\hline Recomendações & Classe & NE & Comentário & $\begin{array}{c}\text { Tabela } \\
2018\end{array}$ & Ref. \\
\hline $\begin{array}{l}\text { Inibidores de SGLT2 (canagliflozina, dapagliflozina ou } \\
\text { empagliflozina) para prevenção de hospitalização por IC em } \\
\text { pacientes com diabetes tipo } 2 \text { e que apresentam fatores } \\
\text { de risco cardiovasculares para aterosclerose ou doença } \\
\text { cardiovascular aterosclerótica estabelecida. }\end{array}$ & I & A & $\begin{array}{c}\text { NOVA: Os ISGLT2 são úteis para reduzir a } \\
\text { hospitalização por insuficiência cardíaca em } \\
\text { pacientes DM2. }\end{array}$ & $\begin{array}{c}\text { Item } 5.2 \\
\text { (página 451) }\end{array}$ & $99-101$ \\
\hline $\begin{array}{l}\text { Inibidores de SGLT2 (dapagliflozina ou empagliflozina) } \\
\text { como medicação antidiabética inicial associada ou não a } \\
\text { metformina em pacientes com ICFEr. }\end{array}$ & I & $A$ & $\begin{array}{l}\text { NOVA: Os iSGLT2 são úteis para tratamento } \\
\text { do diabetes e na redução de evento } \\
\text { cardiovascular e renal. }\end{array}$ & Nova & 102 \\
\hline
\end{tabular}

Os benefícios dos iSGLT2 em pacientes diabéticos tipo 2 (DM2) foram descritos pela primeira vez no estudo EMPA-REG OUTCOME (Empagliflozin, Cardiovascular Outcomes, and Mortality in Type 2 Diabetes), publicado em 2015, que avaliou a empagliflozina em pacientes com DM2, doença cardiovascular estabelecida e recebendo tratamento usual. ${ }^{99}$ Entre os que receberam o fármaco, houve redução significativa dos principais eventos cardiovasculares adversos (MACE = morte CV, IM não fatal ou AVC não fatal) (HR: 0,86 (IC] 95\%: 0,74-0,99), e uma surpreendente redução na hospitalização por insuficiência cardíaca (HIC) (HR: 0,65 (IC 95\%: 0,50-0,85). 0 CANVAS (Canagliflozin and Cardiovascular and Renal Events in Type 2 Diabetes), publicado em 2017, avaliou a canagliflozina em pacientes com DM2 com alto risco para eventos cardiovasculares e recebendo tratamento usual. Houve redução no desfecho primário combinado (MACE = morte CV, IM não fatal ou AVC não fatal) e redução de HIC de 33 \% (HR = 0,67, IC 95\%: 0,52-0,87) bem como dos eventos renais combinados. ${ }^{100} 0$ estudo DECLARE-TIMI 58 (Dapagliflozin and Cardiovascular Outcomes in Type 2 Diabetes) avaliou a dapagliflozina em pacientes com DM2 e doença aterosclerótica estabelecida ou múltiplos fatores de risco para doença aterosclerótica e recebendo tratamento usual. Não houve redução no desfecho primário combinado (MACE = morte CV, infarto do miocárdio ou AVC). Observou-se a redução no desfecho combinado de mortalidade cardiovascular e HIC de 17\%, e de 27\% (HR: 0,73 (IC 95\%: 0,61-0,88) para HIC. ${ }^{101}$ Recentemente, o estudo VERTIS VC (Cardiovascular Outcomes with Ertugliflozin in Type 2 Diabetes) avaliou a ertugliflozina (ainda não comercializada no Brasil) em pacientes com DM2, doença cardiovascular estabelecida e recebendo tratamento usual. Não houve redução no desfecho primário combinado (MACE = morte CV, infarto do miocárdio ou AVC). Foi observado, no entanto, redução de $30 \%$ na HIC. ${ }^{100}$ Tomados em conjunto, os dados disponíveis demonstram a eficácia dos iSGLT2 na redução da incidência de hospitalização por IC em grupos de pacientes com DM2. ${ }^{102}$

AVC: acidente vascular cerebral ; DM2: diabetes tipo 2; HIC: hospitalização por insuficiência cardíaca; IM: infarto do miocárdio; iSGLT2: inibidores do cotransportador de sódio e glicose 2. 


\section{Atualização}

\subsubsection{Disfunção Renal (Tabela 7.5)}

Tabela 7.5 - Recomendações para o uso de inibidores de SGLT2 na prevenção de piora da função renal em pacientes com insuficiência cardíaca com fração de ejeção reduzida (ICFEr)

\begin{tabular}{lccc}
\hline Recomendação & Classe & NE & Comentários \\
\hline $\begin{array}{l}\text { Inibidores de SGLT2 (dapagliflozin ou empagliflozin) em pacientes } \\
\text { com ICFEr para a prevenção da redução da função renal em } \\
\text { pacientes com e sem diabetes, com TGF } \geq 20 \mathrm{~mL} / \mathrm{min} / 1,73 \mathrm{~m}^{2} .\end{array}$ & Ila & A $\begin{array}{c}\text { Tabela } \\
\text { NovA: Os iSGLT2 são úteis para reduzir a } \\
\text { piora progressiva da função renal na ICFEr. }\end{array}$ & $\begin{array}{c}\text { Nova } \\
95,96, \\
98,104\end{array}$ \\
\hline
\end{tabular}

No estudo EMPEROR-Reduced a taxa anual de redução da TFG foi menor com a empagliflozina do que com o placebo (-0,55 versus $-2,28 \mathrm{~mL} / \mathrm{min} / 1,73 \mathrm{~m}{ }^{2}$ por ano, $p<0,001)$ e os pacientes tratados com empagliflozina tiveram menor risco de desfechos renais sérios, independentemente da presença ou ausência de DM2 ${ }^{96}$ Subanálise do DAPA-HF avaliou a eficácia e segurança da dapagliflozina em pacientes com ICFEr, de acordo com a taxa de filtração glomerular (TFG) basal, bem como os efeitos da dapagliflozina na TFG após a randomização. ${ }^{98}$ No estudo $D A P A-H F$, a dapagliflozina não reduziu o desfecho renal composto (RR $=0,71$, IC 95\% 0,44-1,16, $\mathrm{p}=0,17) .{ }^{95}$ Entretanto, em uma subanálise, a taxa de piora da TFG foi menor com a dapagliflozina (-1,09) versus placebo (-2,87), p < 0,001, em pacientes com ou sem DM2. 0 estudo DAPA-CKD (Dapagliflozin in Patients with Chronic Kidney Disease) randomizou 4.304 pacientes com doença renal crônica, com TFG de $25-75 \mathrm{~mL} / \mathrm{min} / 73 \mathrm{~m}^{2}$ e relação albumina-creatinina urinária de $200-5.000$. A dapagliflozina reduziu 0 desfecho primário (composto por redução sustentada da TFG em pelo menos $50 \%$, doença renal terminal ou morte por causas renais ou CV) (9,2\% com dapagliflozina $\times 14,5 \%$ com placebo; $[R R=0,61, I C=9 \%, 0,51-0,72 ; p<0,001]$. Morte ocorreu em 101 (4,5\%) do grupo dapagliflozina $\times 146(6,8 \%)$ grupo placebo $(R R=0,69, I C=95 \% 0,53-0,88, p=0,004)$. A dapagliflozina reduziu a morte cardiovascular ou a hospitalização por insuficiência cardíaca $(0,67$, $0,40-1,13$ versus $0,70,0,52-0,94$, respectivamente, $p$ de interação $=0,88)$. Os resultados foram consistentes, em pacientes com ou sem DM2. ${ }^{104}$ Os dados do EMPEROR-Reduced, DAPA-CKD e da subanálise do DAPA-HF sugerem que o uso dos inibidores de SGLT2 é seguro com ICFEr e com alteração da TFG, independentemente da presença de DM2. Também demonstram que os ISGLT2 podem reduzir a piora da função renal em pacientes com ICFEr.

DM2: diabetes tipo 2; ICFEr: insuficiência cardíaca com fração de ejeção reduzida; iSGLT2: inibidores do cotransportador de sódio e glicose 2; TFG: taxa de filtração glomerular.

\subsubsection{Deficiência de Ferro (Tabela 7.6)}

Tabela 7.6 - Recomendações para uso de ferro intravenoso em pacientes com insuficiência cardíaca com fração de ejeção reduzida (ICFEr)

\begin{tabular}{|c|c|c|c|c|c|}
\hline Recomendação & Classe & NE & Comentários & $\begin{array}{l}\text { Tabela } \\
2018\end{array}$ & Ref. \\
\hline $\begin{array}{l}\text { Reposição intravenosa de carboximaltose férrica em pacientes } \\
\text { com ICFEr e deficiência de ferro (nível ferritina sérica menor } \\
\text { que } 100 \mathrm{ng} / \mathrm{mL} \text { ou entre } 100-299 \mathrm{ng} / \mathrm{mL} \text { com saturação de } \\
\text { transferrina menor que } 20 \% \text { ), mesmo na ausência de anemia } \\
\text { para aumentar capacidade para o exercício, qualidade de vida e } \\
\text { reduzir a hospitalização. }\end{array}$ & Ila & A & Recomendação de 2018 mantida. & $\begin{array}{l}\text { Item } 11.11 \\
\text { (página 470) }\end{array}$ & $\begin{array}{l}\text { Vide } \\
2018\end{array}$ \\
\hline $\begin{array}{l}\text { Reposição intravenosa de carboximaltose férrica em pacientes } \\
\text { com ICFEr, admitidos por IC descompensada com deficiência de } \\
\text { ferro (ferritina sérica menor que } 100 \mathrm{ng} / \mathrm{mL} \text { ou entre } 100-299 \mathrm{ng} / \\
\mathrm{mL} \text {, associada à saturação de transferrina menor que } 20 \% \text { ) após } \\
\text { a estabilização clínica para reduzir readmissão hospitalar. }\end{array}$ & Ila & B & $\begin{array}{l}\text { NOVA: Estudo randomizado multicêntrico } \\
\text { respalda esta recomendação }\end{array}$ & Nova & 105 \\
\hline
\end{tabular}

Em pacientes com IC crônica e deficiência de ferro, o uso de carboximaltose férrica intravenosa demonstrou melhora dos sintomas, da qualidade de vida e redução de hospitalizações em estudos randomizados e metanálises prévias. ${ }^{106-108}$ Recentemente, o estudo AFFIRM-AHF (Ferric carboxymaltose for iron deficiency at discharge after acute heart failure), randomizado, placebo-controlado e multicêntrico avaliou o efeito da carboximaltose férrica intravenosa em 1.132 com ICFEr e deficiência de ferro (estáveis após episódio de descompensação da IC e com deficiência de ferro - ferritina < 100 ng/mL ou ferritina sérica entre $109-299 \mathrm{ng} / \mathrm{mL}$ associada a saturação de transferrina < 20\%) e revelou ser seguro, reduzir hospitalização por IC (217 x 294 hospitalizações; $\mathrm{RR}=0,74 ; 95 \% \mathrm{Cl} 0,58-0,94, \mathrm{p}=0,013)$, embora sem impacto direto em redução de morte cardiovascular. ${ }^{105,109}$

IC: insuficiência cardíaca; ICFEr: insuficiência cardíaca com fração de ejeção reduzida. 
7.5. Algoritmo de Tratamento da Insuficiência Cardíaca com Fração de Ejeção Reduzida (Figura 7.1)

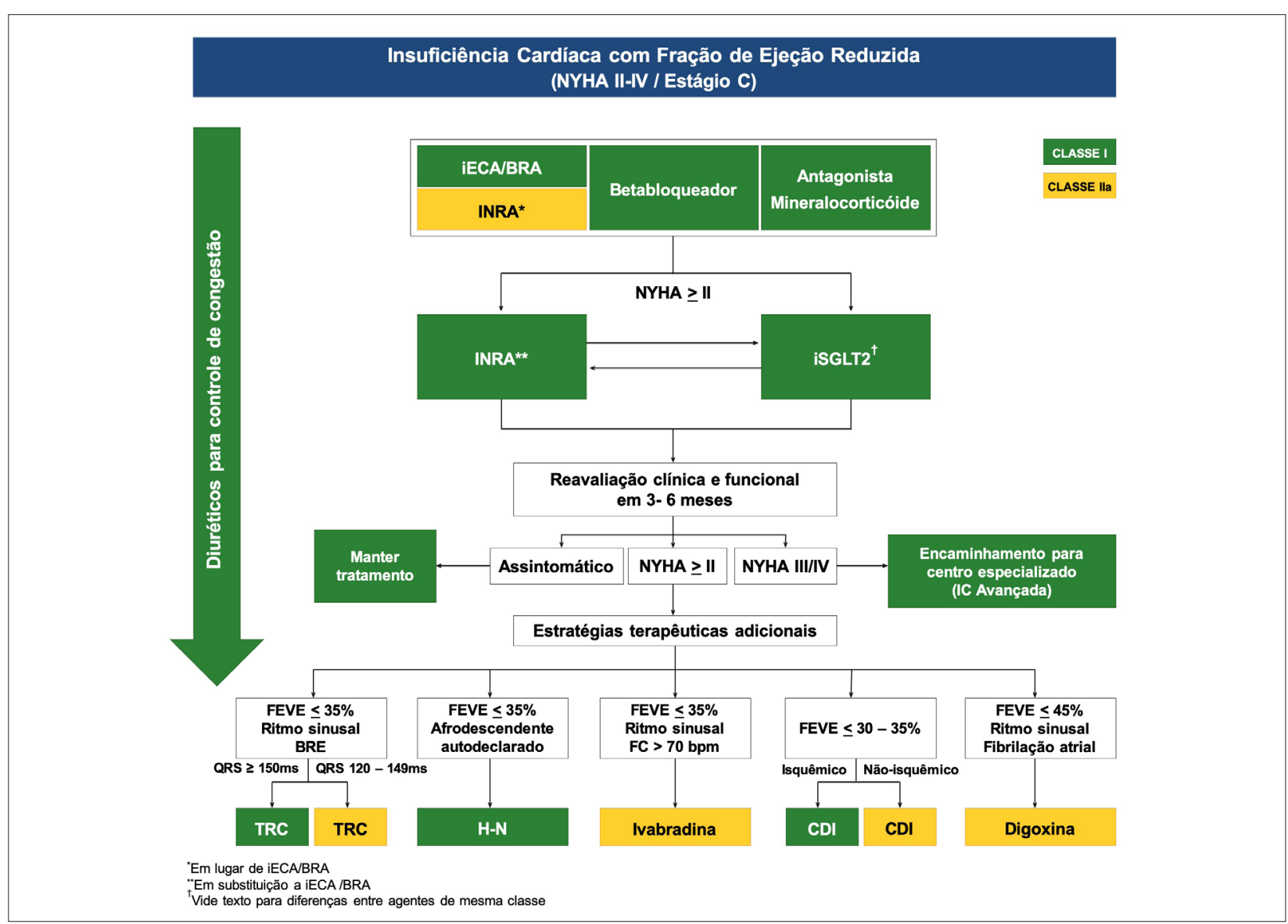

Figura 7.1 - Algoritmo de tratamento da insuficiência cardíaca de fração de ejeção reduzida

BRA: bloqueadores dos receptores da angiotensina II; BRE: bloqueio de ramo esquerdo; CDI: cardiodesfibrilador implantável; FEVE: fração de ejeção do ventrículo esquerdo; H-N: combinação de hidralazina e nitrato; IC: insuficiência cardiaca; iECA: inibidores da enzima conversora da angiotensina; INRA: inibidor da neprilisina e do receptor de angiotensina; iSGLT2: inibidores do cotransportador de sódio e glicose 2; NYHA: New York Heart Association; TRC: terapia de ressincronização cardíaca. 


\section{Atualização}

\section{Inovações em Outros Temas Relacionados à Insuficiência Cardíaca}

8.1. Biomarcadores na Insuficiência Cardíaca com fração de Ejeção Reduzida (Tabela 8.1)

Tabela 8.1 - Recomendações do uso de biomarcadores em pacientes com insuficiência cardíaca com fração de ejeção reduzida (ICFEr)

\begin{tabular}{|c|c|c|c|c|c|}
\hline Recomendações & Classe & NE & Comentário & $\begin{array}{l}\text { Tabela } \\
2018\end{array}$ & Ref. \\
\hline $\begin{array}{l}\text { Dosagem do BNP ou NT-proBNP quando há dúvida no diagnóstico } \\
\text { da IC e como exame de triagem diagnóstica na atenção primária. }\end{array}$ & I & $A$ & Recomendação de 2018 mantida. & $\begin{array}{l}\text { Item } 4.3 \\
\text { (página 451) }\end{array}$ & $\begin{array}{l}\text { Vide } \\
2018\end{array}$ \\
\hline $\begin{array}{l}\text { Dosagem de BNP ou NT-proBNP para estratificação prognóstica em } \\
\text { pacientes com IC. }\end{array}$ & I & A & Recomendação de 2018 mantida. & $\begin{array}{c}\text { Item } 4.3 \\
\text { (página 451) }\end{array}$ & $\begin{array}{l}\text { Vide } \\
2018\end{array}$ \\
\hline $\begin{array}{l}\text { Medidas de BNP ou NT-proBNP como complemento ao exame físico } \\
\text { para avaliar a resposta ao tratamento em pacientes com IC, em caso } \\
\text { de dúvida quanto ao status clínico. }\end{array}$ & Ila & B & $\begin{array}{l}\text { MODIFICADO: Dois estudos recentes, } \\
\text { um randomizado e um observacional } \\
\text { respaldam essa indicação. }\end{array}$ & $\begin{array}{c}\text { Item } 4.3 \\
\text { (página 451) }\end{array}$ & $\begin{array}{l}84, \\
110\end{array}$ \\
\hline $\begin{array}{l}\text { Medidas seriadas de BNP ou NT-proBNP para guiar tratamento, com } \\
\text { alvo do biomarcador a ser atingido. }\end{array}$ & $\mathrm{Ilb}$ & $B$ & $\begin{array}{l}\text { MODIFICADO: Metanálise recente, } \\
\text { que inclui dados do estudo GUIDE-IT, } \\
\text { respaldam a indicação. }\end{array}$ & $\begin{array}{c}\text { Item } 4.3 \\
\text { (página 451) }\end{array}$ & $\begin{array}{l}111 \\
112\end{array}$ \\
\hline
\end{tabular}

Peptídeos natriuréticos podem ser usados para avaliar a resposta a um determinado tratamento. No que diz respeito a esta estratégia, o tratamento é guiado clinicamente e o biomarcador é dosado antes e depois, sem um alvo específico. Novos estudos surgiram, confirmando o que já havia sido mostrado em subanálise do estudo PARADIGM-HF, no qual pacientes que reduziram NT-proBNP abaixo de 1.000 pg/mL, após a introdução de enalapril ou sacubitril-valsartana, apresentaram menos eventos de morte ou hospitalização por IC. ${ }^{113}$ No estudo PIONEER-HF, em pacientes hospitalizados por IC, acompanhados após a alta hospitalar, após 4 semanas se observou uma maior queda de NT-proBNP com sacubitril-valsartana do que com enalapril (46,7\% versus $25,3 \%$ ), e observou-se um menor número de eventos com sacubitril-valsartana. ${ }^{84}$ No estudo PROVE-HF (Association of Change in $N$-Terminal Pro-B-Type Natriuretic Peptide Following Initiation of Sacubitril-Valsartan Treatment With Cardiac Structure and Function in Patients With Heart Failure With Reduced Ejection Fraction), em que pacientes com IC crônica utilizaram sacubitril-valsartana, houve queda significativa de NT-proBNP aos 14 dias de uso da medicação. A queda de NT-proBNP se associou a remodelagem reversa nos 12 meses de seguimento e mostrou menor taxa de eventos. ${ }^{110} \mathrm{~A}$ utilização de peptídeos para guiar o tratamento (com alvo de peptídeo natriurético a ser atingido), diferentemente da situação anterior, é controversa. Apesar dessa estratégia não ter sido superior em comparação ao manuseio convencional no estudo GUIDE-IT (Effect of Natriuretic Peptide-Guided Therapy on Hospitalization or Cardiovascular Mortality in High-Risk Patients With Heart Failure and Reduced Ejection Fraction), existem outros levantamentos prévios que a demonstram. ${ }^{112} 0$ estudo PROTECT ${ }^{114}$ mostrou redução nos eventos cardiovasculares ao se guiar por biomarcadores. Os estudos TIME-CHF (BNPguided vs symptom-guided heart failure therapy) ${ }^{115}$ e Battlescarred ${ }^{116}$ mostraram redução de mortalidade com essa estratégia em pacientes abaixo de 75 anos de idade. Além disso, uma metanálise recente, feita com 4.554 pacientes e incorporando os pacientes do estudo GUIDE-IT, mostrou redução de hospitalizações e mortalidade por todas as causas com o tratamento guiado pelos peptídeos natriuréticos. ${ }^{111}$

IC: insuficiência cardiaca; NT: proBNP-fração N-terminal do peptídeo natriurético atrial do tipo B. 


\subsection{Imunizações na Insuficiência Cardíaca (Tabela 8.2)}

Tabela 8.2 - Recomendações sobre imunização em pacientes com insuficiência cardíaca com fração de ejeção reduzida (ICFEr)

\begin{tabular}{|c|c|c|c|c|c|}
\hline Recomendações & Classe & NE & Comentário & $\begin{array}{c}\text { Tabela } \\
2018\end{array}$ & Ref. \\
\hline $\begin{array}{l}\text { Vacina contra influenza, para prevenção de fatores } \\
\text { agravantes na IC e para redução de mortalidade na IC. }\end{array}$ & 1 & B & $\begin{array}{l}\text { MODIFICADA: Novos estudos retrospectivos } \\
\text { mostram benefício em mortalidade. }\end{array}$ & $\begin{array}{c}\text { Item } 6.7 \\
\text { (página 454) }\end{array}$ & $117-120$ \\
\hline $\begin{array}{l}\text { Vacina contra pneumococos para prevenção de fatores } \\
\text { agravantes na IC. }\end{array}$ & 1 & C & Recomendação de 2018 mantida. & $\begin{array}{c}\text { Item } 6.7 \\
\text { (página 454) }\end{array}$ & Vide 2018 \\
\hline
\end{tabular}

Mais recentemente, não existiam dados sobre o impacto da influenza sobre os desfechos em pacientes com IC. No entanto, estudos populacionais recentes demonstraram a relação entre sazonalidade e maior número de hospitalizações por IC, evidenciado em quatro periodos consecutivos. ${ }^{117}$ Em uma subanálise do estudo PARADIGM-HF, $21 \%$ dos participantes receberam vacinação contra influenza, o que acarretou numa redução de morte total de $19 \%$ após o ajuste de propensão. ${ }^{118} \mathrm{Um}$ estudo de corte dinamarquês feito com 134.048 pacientes com IC, que receberam > 1 vacinação entre 2005 e 2013 , resultou em redução de $18 \%$ na mortalidade por todas as causas; e mais do que 3 vacinações associou-se a redução de $28 \%$ no risco de morte total e $29 \%$ de morte cardiovascular. ${ }^{119} \mathrm{Um}$ estudo com banco de dados de 6.435 pacientes com IC, sendo 695 vacinados antes ou durante 0 inverno de $2017 / 2018$, mostrou redução de $22 \%$ na morte total e de $17 \%$ na morte cardiovascular ou internação por IC. 0 benefício da vacinação sobre a morte total foi maior em pacientes com mais de 70 anos, com redução de mais de $25 \% .{ }^{120}$ Não há estudos sobre o impacto da vacinação para pneumococos. Diversos estudos prospectivos estão em fase de inclusão de pacientes.

IC: insuficiência cardíaca.

8.3. Indicação de Avaliação Genética nas Cardiomiopatias e na Insuficiência Cardíaca (Tabela 8.3)

Tabela 8.3 - Recomendações sobre avaliação genética em pacientes com cardiomiopatias e IC

\begin{tabular}{|c|c|c|c|c|c|}
\hline Recomendações & Classe & NE & Comentário & $\begin{array}{c}\text { Tabela } \\
2018\end{array}$ & Ref. \\
\hline $\begin{array}{l}\text { Aconselhamento genético para pacientes e familiares com } \\
\text { miocardiopatias hereditárias e mutação já identificada. }\end{array}$ & I & C & \multirow{5}{*}{$\begin{array}{l}\text { NOVA: Avanços nas técnicas de avaliação } \\
\text { molecular genética permitem o reconhecimento } \\
\text { precoce de miocardiopatias hereditárias, } \\
\text { favorecendo a subclassificação de síndromes } \\
\text { clínicas e seu tratamento individualizado. }\end{array}$} & Nova & $121-125$ \\
\hline $\begin{array}{l}\text { Rastreamento de familiares em } 10 \text { grau de pacientes com } \\
\text { miocardiopatias hereditárias. }\end{array}$ & I & C & & Nova & $121-125$ \\
\hline $\begin{array}{l}\text { Sequenciamento do gene da transtirretina em pacientes } \\
\text { com diagnóstico de amiloidose cardíaca por deposição de } \\
\text { transtirretina. }\end{array}$ & I & C & & Nova & $121-125$ \\
\hline $\begin{array}{l}\text { Avaliação molecular genética direcionada para avaliação } \\
\text { etiológica e prognóstica de pacientes com fenótipo de } \\
\text { miocardiopatia hereditária. }\end{array}$ & Ila & C & & Nova & $121-125$ \\
\hline $\begin{array}{l}\text { Avaliação molecular genética de rotina para pacientes } \\
\text { com IC. }\end{array}$ & III & C & & Nova & $121-125$ \\
\hline
\end{tabular}

A incorporação do sequenciamento de última geração têm aumentado a sensibilidade dos testes genéticos, possibilitando o diagnóstico precoce com perspectiva de intervenção. ${ }^{121}$ Consequentemente, a avaliação molecular passa a fazer parte da rotina de avaliação de pacientes com miocardiopatias hereditárias, tais como: miocardiopatias hipertróficas, arritmogênica dilatada e/ou restritiva e miocárdio não compactado, em função do seu potencial de fornecer aconselhamento mais individualizado e preciso aos pacientes e familiares com estas doenças. ${ }^{122}$ Um exemplo claro desta necessidade é a diferenciação entre a miocardiopatia amiloidótica por transtirretina (ATTR) do "tipo selvagem" e a familiar: nas formas familiares, é imperativo o rastreamento em cascata de membros da família. É importante ressaltar que, atualmente, terapias utilizadas na ATTR apresentam particular benefício quando iniciadas em fase precoce da doença, conforme descrito no item 2, Tabela $2.4 .{ }^{123}$ Avanços na avaliação prognóstica, envolvendo genes com alto potencial arritmogênico também já foram descritos nas miocardiopatias dilatadas e arritmogênicas. ${ }^{124,125}$ Portanto, fica clara a necessidade de buscar formas mais eficientes da utilização da genética, especialmente no aconselhamento familiar, o que traz resultados seguros e sustentáveis na gestão do cuidado destes pacientes e de suas famílias.

IC: insuficiência cardíaca. 


\section{Perspectivas na insuficiência cardíaca - 9.1. Estimuladores da Guanilato Ciclase (Tabela 9.1) Novas Moléculas}

Tabela 9.1 - Estimuladores da guanilato ciclase no tratamento de pacientes com insuficiência cardíaca com fração de ejeção reduzida (ICFEr)

\begin{tabular}{|c|c|c|c|}
\hline Observações & Comentário & $\begin{array}{c}\text { Tabela } \\
2018\end{array}$ & Ref. \\
\hline $\begin{array}{l}\text { Vericiguat em pacientes com FEVE menor que } 45 \% \text {, NYHA II - } \\
\text { IV para reduzir morbidade, especialmente em pacientes com } \\
\text { hospitalizações frequentes a despeito da terapia clínica otimizada. }\end{array}$ & $\begin{array}{l}\text { POTENCIAL: A observação aqui descrita reflete dados } \\
\text { de estudos recentes com esta nova classe de fármacos, } \\
\text { porém ainda não aprovada pela Anvisa para uso no Brasil. }\end{array}$ & Nova & 126,127 \\
\hline
\end{tabular}

Vericiguat age suprindo o déficit relativo de produção de GMP cíclico que ocorre em pacientes com IC ${ }^{126}$ e foi avaliado em um ensaio clínico randomizado, multicêntrico, controlado por placebo, duplo-cego em pacientes com ICFEr, o estudo VICTORIA (Vericiguat in Patients with Heart Failure and Reduced Ejection Fraction). Este estudo alocou 5.050 pacientes com ICFEr, com FEVE menor que 45\%, em classe II-IV da NYHA, para receber Vericiguat $10 \mathrm{mg} / \mathrm{dia}$, via oral, ou placebo, em adição a todo tratamento clínico. 0 desfecho primário foi morte cardiovascular ou primeira hospitalização por IC. Em um período de 11 meses, o desfecho primário ocorreu em $35,5 \%$ dos pacientes com vericiguat e em $38,5 \%$ dos pacientes tratados com placebo, sendo que 24 pacientes foram necessários para tratar (NNT) e salvar uma vida no período de 11 meses. 0 benefício do desfecho composto deveu-se prioritariamente à redução de hospitalizações, não havendo benefício estatisticamente significativo em morte cardiovascular ou mortalidade total. ${ }^{127}$ Esta medicação tem potencial de integrar o grupo de medicações com efeito sobre sintomas e re-hospitalizações em pacientes com ICFEr, sendo uma opção adicional em pacientes com hospitalizações frequentes a despeito de terapia otimizada e função renal ruim, já que o estudo considerava inclusão de pacientes com TFG maior que $15 \%$ ou com intolerância a outros fármacos. Devemos salientar que esta classe de fármacos é contraindicada em concomitância com nitratos.

FEVE: fração de ejeção do ventrículo esquerdo; IC: insuficiência cardíaca; ICFEr: insuficiência cardíaca com fração de ejeção reduzida; NYHA: New York Heart Association; TFG: taxa de filtração glomerular.

\subsection{Ativador Seletivo da Miosina Cardíaca (Tabela 9.2)}

Tabela 9.2 - Omecamtiv mecarbil no tratamento de pacientes com insuficiência cardíaca com fração de ejeção reduzida (ICFEr)

\begin{tabular}{|c|c|c|c|}
\hline Observações & Comentário & $\begin{array}{c}\text { Tabela } \\
2018\end{array}$ & Ref. \\
\hline $\begin{array}{l}\text { Omecamtiv mecarbil em pacientes com ICFEr aguda } \\
\text { ou crônica. }\end{array}$ & $\begin{array}{l}\text { POTENCIAL: A observação aqui descrita reflete dados de estudos } \\
\text { recentes com esta nova classe de fármacos, porém ainda não aprovada } \\
\text { pela Anvisa para uso no Brasil. }\end{array}$ & Nova & $128-131$ \\
\hline
\end{tabular}

Omecamtiv mercabil é um ativador seletivo da miosina cardíaca e sua ação resulta na ativação e aumento da taxa de hidrólise de ATP, melhorando a contração ventricular em casos de ICFEr. Seu mecanismo de ação é diferente dos tratamentos atuais que bloqueiam a elevada estimulação neuro-hormonal. Os estudos mecanísticos, como o ATOMIC-AHF (Acute Treatment with Omecamtiv Mecarbil to Increase Contractility in Acute Heart Failure) ${ }^{128}$ e o COSMICHF (Chronic Oral Study of Myosin Activation to Increase Contractility in Heart Failure), ${ }^{129}$ mostraram que o fármaco melhora a contratilidade, melhorando a fração de ejeção, o volume ejetado e o débito cardíaco, além de outros parâmetros que indicam melhora da função cardíaca. Os estudos mostraram que promove redução dos níveis de NT-proBNP. Identificou-se também elevação dos níveis de troponina, sem alterações clínicas nos estudos realizados. 0 estudo ATOMIC-AHF, entretanto, em pacientes com IC aguda, não documentou redução da dispneia nos pacientes tratados. No ensaio clínico randomizado GALACTIC-HF, publicado recentemente, pacientes com ICFEr que receberam omecamtiv mecarbil apresentaram menor risco de desfecho composto por evento de IC (definido como hospitalização ou visitas não planejadas por piora da IC) ou morte cardiovascular do que aqueles que receberam placebo. ${ }^{130,131}$ No entanto, quando avaliados individualmente, não houve diferença nos desfechos secundários de morte por todas as causas, morte cardiovascular, primeira hospitalização por IC ou mudança no escore de qualidade de vida Kansas City Cardiomyopathy Questionnaire. 


\section{Referências}

1. O'Meara E, McDonald M, Chan M, Ducharme A, Ezekowitz JA, Giannetti $\mathrm{N}$, et al. CCS/CHFS Heart Failure Guidelines: Clinical Trial Update on Functional Mitral Regurgitation, SGLT2 Inhibitors, ARNI in HFpEF, and Tafamidis in Amyloidosis. Can J Cardiol. 2020;36(2):159-69.

2. Borlaug BA. Evaluation and management of heart failure with preserved ejection fraction. Nat Rev Cardiol. 2020;17(9):559-73.

3. Maisel AS, McCord J, Nowak RM, Hollander JE, Wu AH, Duc P, et al. Bedside B-Type natriuretic peptide in the emergency diagnosis of heart failure with reduced or preserved ejection fraction. Results from the Breathing Not Properly Multinational Study. J Am Coll Cardiol. 2003;41(11):2010-7.

4. Lam CS, Rienstra M, Tay WT, Liu LC, Hummel YM, van der Meer P, et al. Atrial Fibrillation in Heart Failure With Preserved Ejection Fraction: Association With Exercise Capacity, Left Ventricular Filling Pressures, Natriuretic Peptides, and Left Atrial Volume. JACC Heart Fail. 2017;5(2):92-8.

5. Nagueh SF, Smiseth OA, Appleton CP, Byrd BF, Dokainish H, Edvardsen $\mathrm{T}$, et al. Recommendations for the Evaluation of Left Ventricular Diastolic Function by Echocardiography: An Update from the American Society of Echocardiography and the European Association of Cardiovascular Imaging. Eur Heart J Cardiovasc Imaging. 2016;17(12):1321-60.

6. Reddy YNV, Carter RE, Obokata M, Redfield MM, Borlaug BA. A Simple, Evidence-Based Approach to Help Guide Diagnosis of Heart Failure With Preserved Ejection Fraction. Circulation. 2018;138(9):861-70.

7. Pieske B, Tschöpe C, de Boer RA, Fraser AG, Anker SD, Donal E, et al. How to diagnose heart failure with preserved ejection fraction: the HFA-PEFF diagnostic algorithm: a consensus recommendation from the Heart Failure Association (HFA) of the European Society of Cardiology (ESC). Eur J Heart Fail. 2020;22(3):391-412.

8. Barandiarán Aizpurua A, Sanders-van Wijk S, Brunner-La Rocca HP, Henkens M, Heymans S, Beussink-Nelson L, et al. Validation of the HFAPEFF score for the diagnosis of heart failure with preserved ejection fraction. Eur J Heart Fail. 2020;22(3):413-21.

9. Belyavskiy E, Morris DA, Url-Michitsch M, Verheyen N, Meinitzer A, Radhakrishnan AK, et al. Diastolic stress test echocardiography in patients with suspected heart failure with preserved ejection fraction: a pilot study. ESC Heart Fail. 2019;6(1):146-53.

10. Obokata M, Kane GC, Reddy YN, Olson TP, Melenovsky V, Borlaug BA. Role of Diastolic Stress Testing in the Evaluation for Heart Failure With Preserved Ejection Fraction: A Simultaneous Invasive-Echocardiographic Study. Circulation. 2017;135(9):825-38.

11. Lund LH, Claggett B, Liu J, Lam CS, Jhund PS, Rosano GM, et al. Heart failure with mid-range ejection fraction in CHARM: characteristics, outcomes and effect of candesartan across the entire ejection fraction spectrum. Eur J Heart Fail. 2018;20(8):1230-9

12. Solomon SD, Claggett B, Lewis EF, Desai A, Anand I, Sweitzer NK, et al Influence of ejection fraction on outcomes and efficacy of spironolactone in patients with heart failure with preserved ejection fraction. Eur Heart J. 2016;37(5):455-62.

13. Cleland JGF, Bunting KV, Flather MD, Altman DG, Holmes J, Coats AJS, et al. Beta-blockers for heart failure with reduced, mid-range, and preserved ejection fraction: an individual patient-level analysis of double-blind randomized trials. Eur Heart J. 2018;39(1):26-35.

14. Solomon SD, Vaduganathan M, L Claggett B, Packer M, Zile M, Swedberg K, et al. Sacubitril/Valsartan Across the Spectrum of Ejection Fraction in Heart Failure. Circulation. 2020;141(5):352-61.

15. Diretriz Brasileira da Insuficiência Cardíaca Crônica e Aguda. Arq Bras Cardiol. 2018;Arquivos Brasileiros de Cardiologia. 2018;111(3):436-539.

16.. Halliday BP, Wassall R, Lota AS, Khalique Z, Gregson J, Newsome S, et al. Withdrawal of pharmacological treatment for heart failure in patients with recovered dilated cardiomyopathy (TRED-HF): an open-label, pilot, randomised trial. Lancet. 2019;393(10166):61-73.
17. Merlo M, Pyxaras SA, Pinamonti B, Barbati G, Di Lenarda A, Sinagra G Prevalence and prognostic significance of left ventricular reverse remodeling in dilated cardiomyopathy receiving tailored medical treatment. J Am Coll Cardiol. 2011;57(13):1468-76.

18. AJS, Tsutsui $\mathrm{H}$, Abdelhamid CM, Adamopoulos S, Albert N, et al. Universal definition and classification of heart failure: A report of the Heart Failure Society of America, Heart Failure Association of the European Society of Cardiology, Japanese Heart Failure Society and Writing Committee of the Universal Definition of Heart Failure: Endorsed by Canadian Heart Failure Society, Heart Failure Association of India, the Cardiac Society of Australia and New Zealand, and the Chinese Heart Failure Association. Eur J Heart Fail. 2021 Feb 19. Epub ahead of print.

19. Lane T, Fontana M, Martinez-Naharro A, Quarta CC, Whelan CJ, Petrie A et al. Natural History, Quality of Life, and Outcome in Cardiac Transthyretin Amyloidosis. Circulation. 2019;140(1):16-26.

20. González-López E, Gallego-Delgado M, Guzzo-Merello G, de HaroDel Moral FJ, Cobo-Marcos M, Robles C, et al. Wild-type transthyretin amyloidosis as a cause of heart failure with preserved ejection fraction. Eur Heart J. 2015;36(38):2585-94.

21. Cornwell GG, Murdoch WL, Kyle RA, Westermark P, Pitkänen P. Frequency and distribution of senile cardiovascular amyloid. A clinicopathologic correlation. Am J Med. 1983;75(4):618-23.

22. Tanskanen M, Peuralinna T, Polvikoski T, Notkola IL, Sulkava R, Hardy J, et al. Senile systemic amyloidosis affects $25 \%$ of the very aged and associates with genetic variation in alpha2-macroglobulin and tau: a population-based autopsy study. Ann Med. 2008;40(3):232-9

23. Benson MD, Buxbaum JN, Eisenberg DS, Merlini G, Saraiva MJM Sekijima Y, et al. Amyloid nomenclature 2018: recommendations by the International Society of Amyloidosis (ISA) nomenclature committee. Amyloid. 2018;25(4):215-9.

24. Kyle RA, Linos A, Beard CM, Linke RP, Gertz MA, O'Fallon WM, et al. Incidence and natural history of primary systemic amyloidosis in Olmsted County, Minnesota, 1950 through 1989. Blood. 1992;79(7):1817-22

25. Mesquita ET, Jorge AJL, Souza CV, Andrade TR. Cardiac Amyloidosis and its New Clinical Phenotype: Heart Failure with Preserved Ejection Fraction. Arq Bras Cardiol. 2017;109(1):71-80

26. Maurer MS, Elliott P, Comenzo R, Semigran M, Rapezzi C. Addressing Common Questions Encountered in the Diagnosis and Management of Cardiac Amyloidosis. Circulation. 2017;135(14):1357-77.

27. Dorbala S, Cuddy S, Falk RH. How to Image Cardiac Amyloidosis: A Practical Approach. JACC Cardiovasc Imaging. 2020;13(6):1368-83.

28. Maurer MS, SchwartzJH, Gundapaneni B, Elliott PM, Merlini G, WaddingtonCruz M, et al. Tafamidis Treatment for Patients with Transthyretin Amyloid Cardiomyopathy. N Engl J Med. 2018;379(11):1007-16.

29. Lin MH, Yuan WL, Huang TC, Zhang HF, Mai JT, Wang JF. Clinica effectiveness of telemedicine for chronic heart failure: a systematic review and meta-analysis. J Investig Med. 2017;65(5):899-911.

30. Koehler F, Koehler K, Deckwart O, Prescher S, Wegscheider K, Kirwan BA, et al. Efficacy of telemedical interventional management in patients with heart failure (TIM-HF2): a randomised, controlled, parallel-group, unmasked trial. Lancet. 2018;392(10152):1047-57.

31. Abraham WT, Stevenson LW, Bourge RC, Lindenfeld JA, Bauman JG Adamson PB, et al. Sustained efficacy of pulmonary artery pressure to guide adjustment of chronic heart failure therapy: complete follow-up results from the CHAMPION randomised trial. Lancet. 2016;387(10017):453-61.

32. Singhal A, Cowie MR. The Role of Wearables in Heart Failure. Curr Heart Fail Rep. 2020;17(4):125-32.

33. Ahmad T, Wilson FP, Desai NR. The Trifecta of Precision Care in Heart Failure: Biology, Biomarkers, and Big Data. J Am Coll Cardiol. 2018;72(10):1091-4 
34. Choi DJ, Park JJ, Ali T, Lee S. Artificial intelligence for the diagnosis of heart failure. NPJ Digit Med. 2020;3:54

35. Feeny AK, Rickard J, Trulock KM, Patel D, Toro S, Moennich LA, et al. Machine Learning of 12-Lead QRS Waveforms to Identify Cardiac Resynchronization Therapy Patients With Differential Outcomes. Circ Arrhythm Electrophysiol. 2020;13(7):e008210.

36. Stone GW, Lindenfeld J, Abraham WT, Kar S, Lim DS, Mishell JM, et al. Transcatheter Mitral-Valve Repair in Patients with Heart Failure. N Engl J Med. 2018;379(24):2307-18.

37. Tarasoutchi F, Montera MW, Ramos AldO, Sampaio RO, Rosa VEE, Accorsi TAD, et al. Atualização das Diretrizes Brasileiras de Valvopatias 2020. Arq Bras Cardiol. 2020;115(4):720-75.

38. Hunter RJ, Berriman TJ, Diab I, Kamdar R, Richmond L, Baker V, et al. A randomized controlled trial of catheter ablation versus medical treatment of atrial fibrillation in heart failure (the CAMTAF trial). Circ Arrhythm Electrophysiol. 2014;7(1):31-8.

39. Prabhu S, Taylor AJ, Costello BT, Kaye DM, McLellan AJA, Voskoboinik A, et al. Catheter Ablation Versus Medical Rate Control in Atrial Fibrillation and Systolic Dysfunction: The CAMERA-MRI Study. J Am Coll Cardiol. 2017;70(16):1949-61.

40. Khan MN, Jaïs P, Cummings J, Di Biase L, Sanders P, Martin DO, et al. Pulmonary-vein isolation for atrial fibrillation in patients with heart failure. N Engl J Med. 2008;359(17):1778-85

41. Marrouche NF, Kheirkhahan M, Brachmann J. Catheter Ablation for Atrial Fibrillation with Heart Failure. N Engl J Med. 2018;379(5):492.

42. Di Biase L, Mohanty P, Mohanty S, Santangeli P, Trivedi C, Lakkireddy D, et al. Ablation Versus Amiodarone for Treatment of Persistent Atrial Fibrillation in Patients With Congestive Heart Failure and an Implanted Device: Results From the AATAC Multicenter Randomized Trial. Circulation. 2016;133(17):1637-44.

43. Hindricks G, Potpara T, Dagres N, Arbelo E, Bax JJ, Blomström-Lundqvist C, et al. 2020 ESC Guidelines for the diagnosis and management of atrial fibrillation developed in collaboration with the European Association of Cardio-Thoracic Surgery (EACTS). Eur Heart J. 2020 Aug 29;ahead 612 (online ahead print)

44. Willems S, Meyer C, de Bono J, Brandes A, Eckardt L, Elvan A, et al. Cabins, castles, and constant hearts: rhythm control therapy in patients with atrial fibrillation. Eur Heart J. 2019;40(46):3793-9c.

45. Baran DF, Di Biasel E, Brugada J, Hindricks G, Maggioni AP, Tavazzi L, Vardas P, et al. The atrial fibrillation ablation pilot study: a European Survey on Methodology and results of catheter ablation for atrial fibrillation conducted by the European Heart Rhythm Association. Eur Heart J. 2014;35(22):1466-78

46. The European Society for Cardiology. ESC Guidance for the Diagnosis and Management of CV Disease during the COVID-19 Pandemic. Available from:https://www.escardio.org/Education/COVID-19-and-Cardiology/ ESC-COVID-19-Guidance. (Last update: 10 June 2020).

47. Panjrath GS, Krepp J. COVID-19 and Heart Failure: Harsh Reality of PreExisting Conditions. J Am Coll Cardiol. 2020;76(20):2349-51.

48. Alvarez-Garcia J, Lee S, Gupta A, Cagliostro M, Joshi AA, Rivas-Lasarte M, et al. Prognostic Impact of Prior Heart Failure in Patients Hospitalized With COVID-19. J Am Coll Cardiol. 2020;76(20):2334-48.

49. Bozkurt B, Kovacs R, Harrington B. Joint HFSA/ACC/AHA Statement Addresses Concerns Re: Using RAAS Antagonists in COVID-19. J Card Fail. 2020;26(5):370

50. Reynolds HR, Adhikari S, Pulgarin C, Troxel AB, Iturrate E, Johnson SB, et al. Renin-Angiotensin-Aldosterone System Inhibitors and Risk of Covid-19. N Engl J Med. 2020;382(25):2441-8.

51. Defilippis EM, Reza N, Donald E, Givertz MM, Lindenfeld J, Jessup M. Considerations for Heart Failure Care During the COVID-19 Pandemic. JACC Heart Fail. 2020;8(8):681-91.
52. Gorodeski EZ, Goyal P, Cox ZL, Thibodeau JT, Reay RE, Rasmusson K, et al. Virtual Visits for Care of Patients with Heart Failure in the Era of COVID-19: A Statement from the Heart Failure Society of America. J Card Fail. 2020;26(6):448-56.

53. Givertz MM, Stevenson LW, Costanzo MR, Bourge RC, Bauman JG, Ginn G, et al. Pulmonary Artery Pressure-Guided Management of Patients With Heart Failure and Reduced Ejection Fraction. J Am Coll Cardiol. 2017;70(15):1875-86

54. Desai AS, Bhimaraj A, Bharmi R, Jermyn R, Bhatt K, Shavelle D, et al. Ambulatory Hemodynamic Monitoring Reduces Heart Failure Hospitalizations in "Real-World" Clinical Practice. J Am Coll Cardiol. 2017;69(19):2357-65.

55. Schmier JK, Ong KL, Fonarow GC. Cost-Effectiveness of Remote Cardiac Monitoring With the CardioMEMS Heart Failure System. Clin Cardiol. 2017;40(7):430-6.

56. Angermann CE, Assmus B, Anker SD, Asselbergs FW, Brachmann J, BrettME, et al. Pulmonary artery pressure-guided therapy in ambulatory patients with symptomatic heart failure: the CardioMEMS European Monitoring Study for Heart Failure (MEMS-HF). Eur J Heart Fail. 2020;22(10):1891-901.

57. Shavelle DM, Desai AS, Abraham WT, Bourge RC, Raval N, Rathman LD, et al. Lower Rates of Heart Failure and All-Cause Hospitalizations During Pulmonary Artery Pressure-Guided Therapy for Ambulatory Heart Failure: One-Year Outcomes From the CardioMEMS Post-Approval Study. Circ Heart Fail. 2020;13(8):e006863.

58. Baran DA, Grines CL, Bailey S, Burkhoff D, Hall SA, Henry TD, et al. SCAI clinical expert consensus statement on the classification of cardiogenic shock: This document was endorsed by the American College of Cardiology (ACC), the American Heart Association (AHA), the Society of Critical Care Medicine (SCCM), and the Society of Thoracic Surgeons (STS) in April 2019. Catheter Cardiovasc Interv. 2019;94(1):29-37.

59. Jentzer JC, van Diepen S, Barsness GW, Henry TD, Menon V, Rihal CS, et al. Cardiogenic Shock Classification to Predict Mortality in the Cardiac Intensive Care Unit. J Am Coll Cardiol. 2019;74(17):2117-28.

60. Tehrani BN, Truesdell AG, Psotka MA, Rosner C, Singh R, Sinha SS, et al. A Standardized and Comprehensive Approach to the Management of Cardiogenic Shock. JACC Heart Fail. 2020;8(11):879-91.

61. Garan AR, Kanwar M, Thayer KL, Whitehead E, Zweck E, HernandezMontfort J, et al. Complete Hemodynamic Profiling With Pulmonary Artery Catheters in Cardiogenic Shock Is Associated With Lower In-Hospital Mortality. JACC Heart Fail. 2020;8(11):903-13.

62. Shah MR, Hasselblad V, Stevenson LW, Binanay C, O'Connor CM, Sopko $\mathrm{G}$, et al. Impact of the pulmonary artery catheter in critically ill patients: meta-analysis of randomized clinical trials. JAMA. 2005;294(13):1664-70.

63. Sotomi Y, Sato N, Kajimoto K, Sakata Y, Mizuno M, Minami Y, et al. Impact of pulmonary artery catheter on outcome in patients with acute heart failure syndromes with hypotension or receiving inotropes: from the ATTEND Registry. Int J Cardiol. 2014;172(1):165-72.

64. Binanay C, Califf RM, Hasselblad V, O'Connor CM, Shah MR, Sopko G, et al. Evaluation study of congestive heart failure and pulmonary artery catheterization effectiveness: the ESCAPE trial. JAMA. 2005;294(13):1625-33.

65. Metra M, Ponikowski P, Dickstein K, McMurray M, Dinatolo E, Dasseni N. et al. Advanced chronic heart failure :a position statement from the study Group on Advanced Heart Failure of the Heart Failure Association of the European Society of Cardiology. Eur Heart J. 2007;; 9(6-7):684-94.

66. Metra M, Dinatolo E, Dasseni N. The New Heart Failure Association Definition of Advanced Heart Failure. Card Fail Rev. 2019;5(1):5-8.

67. Crespo-Leiro MG, Metra M, Lund LH, Milicic D, Costanzo MR, Filippatos G, et al. Advanced heart failure: a position statement of the Heart Failure Association of the European Society of Cardiology. Eur J Heart Fail. 2018;20(11):1505-35 
68. Truby LK, Rogers JG,. Advanced heart failure:epidemiology,diagnosis and therapeutic approaches. JACC Heart Fail. 2020;8(7):523-36.

69. Al-Fares AA, Randhawa VK, Englesakis M, McDonald MA, Nagpal AD, Estep JD, et al. Optimal Strategy and Timing of Left Ventricular Venting During Veno-Arterial Extracorporeal Life Support for Adults in Cardiogenic Shock: A Systematic Review and Meta-Analysis. Circ Heart Fail. 2019;12(11):e006486.

70. Russo JJ, Aleksova N, Pitcher I, Couture E, Parlow S, Faraz M, et al. Left Ventricular Unloading During Extracorporeal Membrane Oxygenation in Patients With Cardiogenic Shock. J Am Coll Cardiol. 2019;73(6):654-62.

71. Piechura LM, Coppolino A, Mody GN, Rinewalt DE, Keshk M, Ogawa M, et al. Left ventricle unloading strategies in ECMO: A single-center experience. J Card Surg. 2020;35(7):1514-24

72. Cevasco M, Takayama H, Ando M, Garan AR, Naka Y, Takeda K. Left ventricular distension and venting strategies for patients on venoarterial extracorporeal membrane oxygenation. J Thorac Dis. 2019;11(4):1676-83.

73. Pan P, Yan P, Liu D, Wang X, Zhou X, Long Y, et al. Outcomes of VA-ECMO with and without Left Centricular (LV) Decompression Using Intra-Aortic Balloon Pumping (IABP) versus Other LV Decompression Techniques: A Systematic Review and Meta-Analysis. Med Sci Monit. 2020;26:e924009.

74. Guglin M, Zucker MJ, Bazan VM, Bozkurt B, El Banayosy A, Estep JD, et al. Venoarterial ECMO for Adults: JACC Scientific Expert Panel. J Am Coll Cardiol. 2019;73(6):698-716.

75. Stephens AF, Wanigasekara D, Pellegrino VA, Burrell AJC, Marasco SF, Kaye DM, et al. Comparison of Circulatory Unloading Techniques for Venoarterial Extracorporeal Membrane Oxygenation. ASAIO J. 2020Oct 14; (online ahead print).

76. Silvetti S, Nieminen MS. Repeated or intermittent levosimendan treatment in advanced heart failure: An updated meta-analysis. Int J Cardiol. 2016;202:138-43.

77. Silvetti S, Belletti A, Fontana A, Pollesello P. Rehospitalization after intermittent levosimendan treatment in advanced heart failure patients: a meta-analysis of randomized trials. ESC Heart Fail. 2017;4(4):595-604

78. Nizamic T, Murad MH, Allen LA, Mcllvennan CK, Wordingham SE, Matlock DD, et al. Ambulatory Inotrope Infusions in Advanced Heart Failure: A Systematic Review and Meta-Analysis. JACC Heart Fail. 2018;6(9):757-67.

79. Comín-Colet J, Manito N, Segovia-Cubero J, Delgado J, García Pinilla $J \mathrm{M}$, Almenar L, et al. Efficacy and safety of intermittent intravenous outpatient administration of levosimendan in patients with advanced heart failure: the LION-HEART multicentre randomised trial. Eur J Heart Fail. 2018:20(7):1128-36.

80. Granger CB, McMurray JJ, Yusuf S, Held P, Michelson EL, Olofsson B, et al. Effects of candesartan in patients with chronic heart failure and reduced leftventricular systolic function intolerant to angiotensin-converting-enzyme inhibitors: the CHARM-Alternative trial. Lancet. 2003;362(9386):772-6.

81. Pitt B, Zannad F, Remme WJ, Cody R, Castaigne A, Perez A, et al. The effect of spironolactone on morbidity and mortality in patients with severe heart failure. Randomized Aldactone Evaluation Study Investigators. N Engl J Med. 1999;341(10):709-17.

82. Zannad F, McMurray JJ, Krum H, van Veldhuisen DJ, Swedberg K, Shi H, et al. Eplerenone in patients with systolic heart failure and mild symptoms. $\mathrm{N}$ Engl J Med. 2011;364(1):11-21

83. McMurray JJ, Packer M, Desai AS, Gong J, Lefkowitz MP, Rizkala AR, et al. Angiotensin-neprilysin inhibition versus enalapril in heart failure. N Engl J Med. 2014:371(11):993-1004.

84. Velazquez EJ, Morrow DA, DeVore AD, Duffy Cl, Ambrosy AP, McCague $\mathrm{K}$, et al. Angiotensin-Neprilysin Inhibition in Acute Decompensated Heart Failure. N Engl J Med. 2019;380(6):539-48.

85. Effect of metoprolol CR/XL in chronic heart failure: Metoprolol CR/XL Randomised Intervention Trial in Congestive Heart Failure (MERIT-HF). Lancet. 1999;353(9169):2001-7.
86. The Cardiac Insufficiency Bisoprolol Study II (CIBIS-II): a randomised trial. Lancet. 1999;353(9146):9-13.

87. Packer M, Fowler MB, Roecker EB, Coats AJ, Katus HA, Krum H, et al. Effect of carvedilol on the morbidity of patients with severe chronic heart failure: results of the carvedilol prospective randomized cumulative survival (COPERNICUS) study. Circulation. 2002;106(17):2194-9.

88. SOLVD Investigators. Effect of enalapril on mortality and the development of heart failure in asymptomatic patients with reduced left ventricular ejection fractions. N Engl J Med. 1992;327(10):685-91.

89. Taylor AL, Ziesche S, Yancy C, Carson P, D'Agostino R, Ferdinand K, et al Combination of isosorbide dinitrate and hydralazine in blacks with heart failure. N Engl J Med. 2004;351(20):2049-57.

90. Swedberg K, Komajda M, Böhm M, Borer JS, Ford I, Dubost-Brama A, et al. Ivabradine and outcomes in chronic heart failure (SHIFT): a randomised placebo-controlled study. Lancet. 2010;376(9744):875-85.

91. Digitalis Investigation Group. The effect of digoxin on mortality and morbidity in patients with heart failure. N Engl J Med. 1997;336(8):525-33.

92. DeVore AD, Braunwald E, Morrow DA, Duffy Cl, Ambrosy AP, Chakraborty $\mathrm{H}$, et al. Initiation of Angiotensin-Neprilysin Inhibition After Acute Decompensated Heart Failure: Secondary Analysis of the Open-label Extension of the PIONEER-HF Trial. JAMA Cardiol. 2020;5(2):202-7.

93. Wachter R, Senni M, BelohlavekJ, Straburzynska-Migaj E, Witte KK, Kobalava $Z$, et al. Initiation of sacubitril/valsartan in haemodynamically stabilised heart failure patients in hospital or early after discharge: primary results of the randomised TRANSITION study. Eur J Heart Fail. 2019;21(8):998-1007.

94. Anger AP, Braunwald E, Morrow DA, DeVore AD, McCague K, Meng X, et al. Angiotensin Receptor-Neprilysin Inhibition Based on History of Heart Failure and Use of Renin-Angiotensin System Antagonists. J Am Coll Cardiol. 2020;76(9):1034-48

95. McMurray JJV, Solomon SD, Inzucchi SE, Køber L, Kosiborod MN, Martinez FA, et al. Dapagliflozin in Patients with Heart Failure and Reduced Ejection Fraction. N Engl J Med.2019;381(21):1995-2008.

96. Packer M, Anker SD, Butler J, Filippatos G, Pocock SJ, Carson P, et al Cardiovascular and Renal Outcomes with Empagliflozin in Heart Failure. N Engl J Med. 2020;383(15):1413-24.

97. Zannad F, Ferreira JP, Pocock SJ, Anker SD, Butler J, Filippatos G, et al. SGLT2 inhibitors in patients with heart failure with reduced ejection fraction: a meta-analysis of the EMPEROR-Reduced and DAPA-HF trials. Lancet. 2020:396(10254):819-29.

98. Jhund PS, Solomon SD, Docherty KF, Heerspink HJL, Anand IS, Böhm M et al. Efficacy of Dapagliflozin on Renal Function and Outcomes in Patients with Heart Failure with Reduced Ejection Fraction: Results of DAPA-HF. Circulation. 2021:143(4):298-309.

99. Zinman B, Wanner C, Lachin JM, Fitchett D, Bluhmki E, Hantel S, et al Empagliflozin, Cardiovascular Outcomes, and Mortality in Type 2 Diabetes. N Engl J Med. 2015;373(22):2117-28.

100. Neal B, Perkovic V, Mahaffey KW, de Zeeuw D, Fulcher G, Erondu N, et al Canagliflozin and Cardiovascular and Renal Events in Type 2 Diabetes. N Engl J Med. 2017;377(7):644-57.

101. Wiviott SD, Raz I, Bonaca MP, Mosenzon O, Kato ET, Cahn A, et al. Dapagliflozin and Cardiovascular Outcomes in Type 2 Diabetes. N Engl J Med. 2019;380(4):347-57.

102.Zelniker TA, Wiviott SD, Raz I, Im K, Goodrich EL, Bonaca MP, et al. SGLT2 inhibitors for primary and secondary prevention of cardiovascular and renal outcomes in type 2 diabetes: a systematic review and meta-analysis of cardiovascular outcome trials. Lancet. 2019;393(10166):31-9.

103. Cannon CP, Pratley R, Dagogo-Jack S, Mancuso J, Huyck S, Masiukiewicz $\mathrm{U}$, et al. Cardiovascular Outcomes with Ertugliflozin in Type 2 Diabetes. N Engl J Med. 2020;383(15):1425-35. 
104. Hunter HJL, Stefánsson BV, Correa-Rotter R, Chertow GM, Greene T, Hou FF, et al. Dapagliflozin in Patients with Chronic Kidney Disease. N Engl J Med. 2020;383(15):1436-46.

105. Ponikowski P, Kirwan BA, Anker SD, McDonagh T, Dorobantu M, Drozdz J, et al. Ferric carboxymaltose for iron deficiency at discharge after acute heart failure: a multicentre, double-blind, randomised, controlled trial. Lancet. 2020;396(10266):1895-904.

106. Ponikowski P, van Veldhuisen DJ, Comin-Colet J, Ertl G, Komajda M, Mareev V, et al. Beneficial effects of long-term intravenous iron therapy with ferric carboxymaltose in patients with symptomatic heart failure and iron deficiencyt. Eur HeartJ. 2015;36(11):657-68.

107. Beck-da-Silva L, Piardi D, Soder S, Rohde LE, Pereira-Barretto AC, de Albuquerque D, etal. IRON-HF study: a randomized trial to assess the effects of iron in heart failure patients with anemia. IntJ Cardiol. 2013;168(4):3439-42.

108. Anker SD, Kirwan BA, van Veldhuisen DJ, Filippatos G, Comin-Colet J, Ruschitzka F, et al. Effects of ferric carboxymaltose on hospitalisations and mortality rates in iron-deficient heart failure patients: an individual patient data meta-analysis. Eur J Heart Fail. 2018;20(1):125-33.

109. Ponikowski P, Kirwan BA, Anker SD, Dorobantu M, Drozdz J, Fabien V, et al. Rationale and design of the AFFIRM-AHF trial: a randomised, doubleblind, placebo-controlled trial comparing the effect of intravenous ferric carboxymaltose on hospitalisations and mortality in iron-deficient patients admitted for acute heart failure. Eur J Heart Fail. 2019;21(12):1651-8.

110. Januzzi JL, Prescott MF, Butler J, Felker GM, Maisel AS, McCague K, et al. Association of Change in N-Terminal Pro-B-Type Natriuretic Peptide Following Initiation of Sacubitril-Valsartan Treatment With Cardiac Structure and Function in Patients With Heart Failure With Reduced Ejection Fraction. JAMA. 2019;322(11):1-11.

111. McLellan J, Bankhead CR, Oke JL, Hobbs FDR, Taylor CJ, Perera R. Natriuretic peptide-guided treatment for heart failure: a systematic review and meta-analysis. BMJ Evid Based Med. 2020;25(1):33-7.

112. Felker GM, Anstrom KJ, Adams KF, Ezekowitz JA, Fiuzat M, Houston-Miller $\mathrm{N}$, et al. Effect of Natriuretic Peptide-Guided Therapy on Hospitalization or Cardiovascular Mortality in High-Risk Patients With Heart Failure and Reduced Ejection Fraction: A Randomized Clinical Trial. JAMA. 2017;318(8):713-20.

113. Zile MR, Claggett BL, Prescott MF, McMurray JJ, Packer M, Rouleau JL, et al. Prognostic Implications of Changes in N-Terminal Pro-B-Type Natriuretic Peptide in Patients With Heart Failure. J Am Coll Cardiol. 2016;68(22):2425-36.

114. Januzzi JL, Rehman SU, Mohammed AA, Bhardwaj A, Barajas L, Barajas J, et al. Use of amino-terminal pro-B-type natriuretic peptide to guide outpatient therapy of patients with chronic left ventricular systolic dysfunction. J Am Coll Cardiol. 2011;58(18):1881-9.

115. Pfisterer M, Buser P, Rickli H, Gutmann M, Erne P, Rickenbacher P, et al. BNPguided vs symptom-guided heart failure therapy: the Trial of Intensified vs Standard Medical Therapy in Elderly Patients With Congestive Heart Failure (TIME-CHF) randomized trial. JAMA. 2009;301(4):383-92.

116. Lainchbury JG, Troughton RW, Strangman KM, Frampton CM, Pilbrow A, Yandle TG, et al. N-terminal pro-B-type natriuretic peptide-guided treatment for chronic heart failure: results from the BATTLESCARRED (NTproBNP-Assisted Treatment To Lessen Serial Cardiac Readmissions and Death) trial. J Am Coll Cardiol. 2009;55(1):53-60.

117. Kytömaa S, Hegde S, Claggett B, Udell JA, Rosamond W, Temte J, et al. Association of Influenza-like Illness Activity With Hospitalizations for Heart
Failure: The Atherosclerosis Risk in Communities Study. JAMA Cardiol. 2019;4(4):363-9.

118. Vardeny O, Claggett B, Udell JA, Packer M, Zile M, Rouleau J, et al. Influenza Vaccination in Patients With Chronic Heart Failure: The PARADIGM-HF Trial. JACC Heart Fail. 2016;4(2):152-8.

119. Modin D, Jørgensen ME, Gislason G, Jensen JS, Køber L, Claggett B, et al. Influenza Vaccine in Heart Failure. Circulation. 2019;139(5):575-86.

120. Gotsman I, Shuvy M, Tahiroglu I, Zwas DR, Keren A. Influenza vaccination and outcome in heart failure. Am J Cardiol. 2020;128:134-9.

121. Musunuru K, Hershberger RE, Day SM, Klinedinst NJ, Landstrom AP, Parikh VN, et al. Genetic Testing for Inherited Cardiovascular Diseases: A Scientific Statement From the American Heart Association. Circ Genom Precis Med. 2020;13(4):e000067.

122. Charron P, Arad M, Arbustini E, Basso C, Bilinska Z, Elliott P, et al. Genetic counselling and testing in cardiomyopathies: a position statement of the European Society of Cardiology Working Group on Myocardial and Pericardial Diseases. Eur Heart J. 2010;31(22):2715-26.

123. Kittleson MM, Maurer MS, Ambardekar AV, Bullock-Palmer RP, Chang PP, Eisen HJ, et al. Cardiac Amyloidosis: Evolving Diagnosis and Management: A Scientific Statement From the American Heart Association. Circulation. 2020;142(1):e7-e22.

124. Towbin JA, McKenna WJ, Abrams DJ, Ackerman MJ, Calkins H, Darrieux FCC, et al. 2019 HRS expert consensus statement on evaluation, risk stratification, and management of arrhythmogenic cardiomyopathy: Executive summary. Heart Rhythm. 2019;16(11):e373-e407.

125. Pinto YM, Elliott PM, Arbustini E, Adler Y, Anastasakis A, Böhm M, et al. Proposal for a revised definition of dilated cardiomyopathy, hypokinetic non-dilated cardiomyopathy, and its implications for clinical practice: a position statement of the ESC working group on myocardial and pericardial diseases. Eur Heart J. 2016;37(23):1850-8.

126. Armstrong PW, Roessig L, Patel MJ, Anstrom KJ, Butler J, Voors AA, et al. A Multicenter, Randomized, Double-Blind, Placebo-Controlled Trial of the Efficacy and Safety of the Oral Soluble Guanylate Cyclase Stimulator: The VICTORIA Trial. JACC Heart Fail. 2018;6(2):96-104.

127. Armstrong PW, Pieske B, Anstrom KJ, Ezekowitz J, Hernandez AF, Butler J, et al. Vericiguat in Patients with Heart Failure and Reduced Ejection Fraction. N Engl J Med. 2020;382(20):1883-93.

128. Teerlink JR, Felker GM, McMurray JJV, Ponikowski P, Metra M, Filippatos GS, et al. Acute Treatment With Omecamtiv Mecarbil to Increase Contractility in Acute Heart Failure: The ATOMIC-AHF Study. J Am Coll Cardiol. 2016;67(12):1444-55.

129. Teerlink JR, Felker GM, McMurray JJ, Solomon SD, Adams KF, Cleland JG, et al. Chronic Oral Study of Myosin Activation to Increase Contractility in Heart Failure (COSMIC-HF): a phase 2, pharmacokinetic, randomised, placebo-controlled trial. Lancet. 2016;388(10062):2895-903.

130. Teerlink JR, Diaz R, Felker GM, McMurray JJV, Metra M, Solomon SD, et al. Omecamtiv Mecarbil in Chronic Heart Failure With Reduced Ejection Fraction: Rationale and Design of GALACTIC-HF. JACC Heart Fail. 2020;8(4):329-40.

131. Teerlink JR, Diaz R, Felker GM, McMurray JJV, Metra M, Solomon SD, et al. Cardiac Myosin Activation with Omecamtiv Mecarbil in Systolic Heart Failure. N Engl J Med. 2020;384(2):105-16. 


Atualização de Tópicos Emergentes da Diretriz Brasileira de Insuficiência Cardíaca - 2021

0 relatório abaixo lista as declarações de interesse conforme relatadas à SBC

pelos especialistas durante o período de desenvolvimento desta atualização, 2020.

Especialista Tipo de relacionamento com a indústria

Aguinaldo F. Freitas Jr. Declaração financeira

A - Pagamento de qualquer espécie e desde que economicamente apreciáveis, feitos a (i) você, (ii) ao seu cônjuge/ companheiro ou a qualquer outro membro que resida com você, (iii) a qualquer pessoa jurídica em que qualquer destes seja controlador, sócio, acionista ou participante, de forma direta ou indireta, recebimento por palestras, aulas, atuação como proctor de treinamentos, remunerações, honorários pagos por participações em conselhos consultivos, de investigadores, ou outros comitês, etc. Provenientes da indústria farmacêutica, de órteses, próteses, equipamentos e implantes, brasileiras ou estrangeiras:

- Novartis: Insuficiência cardíaca; Servier: Insuficiência cardíaca; Bayer: Anticoagulação.

\begin{tabular}{ll}
\hline Andréia Biolo & Nada a ser declarado \\
\hline Antonio Carlos Pereira Barretto & Outros relacionamentos
\end{tabular}

Financiamento de atividades de educação médica continuada, incluindo viagens, hospedagens e inscrições para congressos e cursos, provenientes da indústria farmacêutica, de órteses, próteses, equipamentos e implantes, brasileiras ou estrangeiras:

- Novartis: sacubitril valsartana; Astrazeneca: dapagliflozina, succinato de metoprolol, candesartana; Servier: ivabradina.

Antônio José Lagoeiro Jorge

\section{Outros relacionamentos}

Financiamento de atividades de educação médica continuada, incluindo viagens, hospedagens e inscrições para congressos e cursos, provenientes da indústria farmacêutica, de órteses, próteses, equipamentos e implantes, brasileiras ou estrangeiras:

- Novartis: Entresto.

\begin{tabular}{ll}
\hline Bruno Biselli & Nada a ser declarado \\
\hline Carlos Eduardo Lucena Montenegro & Declaração financeira \\
A - Pagamento de qualq
\end{tabular}

A - Pagamento de qualquer espécie e desde que economicamente apreciáveis, feitos a (i) você, (ii) ao seu cônjuge/companheiro ou a qualquer outro membro que resida com você, (iii) a qualquer pessoa jurídica em que qualquer destes seja controlador, sócio, acionista ou participante, de forma direta ou indireta, recebimento por palestras, aulas, atuação como proctor de treinamentos, remunerações, honorários pagos por participações em conselhos consultivos, de investigadores, ou outros comitês, etc. Provenientes da indústria farmacêutica, de órteses, próteses, equipamentos e implantes, brasileiras ou estrangeiras:

- Astrazeneca: palestras; Novartis: palestras; Servier: palestras; Merck: palestras.

Outros relacionamentos

Financiamento de atividades de educação médica continuada, incluindo viagens, hospedagens e inscrições para congressos e cursos, provenientes da indústria farmacêutica, de órteses, próteses, equipamentos e implantes, brasileiras ou estrangeiras:

- Pfizer: Viagem para Congresso; AstraZeneca: Viagem para Simpósio; Novartis: Viagem para Simpósio.

Denilson Campos de Albuquerque Declaração financeira

B - Financiamento de pesquisas sob sua responsabilidade direta/pessoal (direcionado ao departamento ou instituição) provenientes da indústria farmacêutica, de órteses, próteses, equipamentos e implantes, brasileiras ou estrangeiras:

- FAPERJ: Pesquisa Clínica; Boehringer: Participante de Pesquisa Clínica Multicêntrica Internacional.

\begin{tabular}{ll}
\hline Dirceu Rodrigues de Almeida & Nada a ser declarado \\
\hline Edimar Alcides Bocchi & Declaração financeira \\
& A - Pagamento de qualquer espécie e desde que economicamente apreciáveis, feitos a (i) você, (ii) ao seu \\
& cônjuge/companheiro ou a qualquer outro membro que resida com você, (iii) a qualquer pessoa jurídica em que \\
& qualquer destes seja controlador, sócio, acionista ou participante, de forma direta ou indireta, recebimento por \\
& palestras, aulas, atuação como proctor de treinamentos, remunerações, honorários pagos por participações em \\
& conselhos consultivos, de investigadores, ou outros comitês, etc. Provenientes da indústria farmacêutica, de \\
& órteses, próteses, equipamentos e implantes, brasileiras ou estrangeiras: \\
& - AstraZeneca: ISGLT2; Bayer: ISGLT2, Vericiguat; Boehringer: ISGLT2.
\end{tabular}

\begin{tabular}{ll}
\hline Edval Gomes dos Santos Júnior & Nada a ser declarado \\
\hline Estêvão Lanna Figueiredo & Declaração financeira \\
& B - Financiamento de pesquisas sob sua responsabilidade direta/pessoal (direcionado ao departamento ou \\
& instituição) provenientes da indústria farmacêutica, de órteses, próteses, equipamentos e implantes, brasileiras ou \\
& estrangeiras: \\
& - AstraZeneca: Dapagliflozina; Boehringer: Empagliflozina; Pfizer: Apixabana; Novartis. \\
\hline Evandro Tinoco Mesquita & Outros relacionamentos \\
& Vínculo empregatício com a indústria farmacêutica, de órteses, próteses, \\
& Equipamentos e implantes, brasileiras ou estrangeiras, assim como se tem \\
& Relação vínculo empregatício com operadoras de planos de saúde ou em \\
& Auditorias médicas (incluindo meio período) durante o ano para o qual você está declarando: \\
& - UnitedHealth Group.
\end{tabular}




\section{Atualização}

Fabiana G. Marcondes-Braga
Declaração financeira

A - Pagamento de qualquer espécie e desde que economicamente apreciáveis, feitos a (i) você, (ii) ao seu cônjuge/ companheiro ou a qualquer outro membro que resida com você, (iii) a qualquer pessoa jurídica em que qualquer destes seja controlador, sócio, acionista ou participante, de forma direta ou indireta, recebimento por palestras, aulas, atuação como proctor de treinamentos, remunerações, honorários pagos por participações em conselhos consultivos, de investigadores, ou outros comitês, etc. Provenientes da indústria farmacêutica, de órteses, próteses, equipamentos e implantes, brasileiras ou estrangeiras: - Novartis: Palestras; AstraZeneca: Palestras e Conselho Consultivo; Boehringer: Conselho Consultivo.

Fábio Fernandes Declaração financeira

A - Pagamento de qualquer espécie e desde que economicamente apreciáveis, feitos a (i) você, (ii) ao seu cônjuge/ companheiro ou a qualquer outro membro que resida com você, (iii) a qualquer pessoa jurídica em que qualquer destes seja controlador, sócio, acionista ou participante, de forma direta ou indireta, recebimento por palestras, aulas, atuação como proctor de treinamentos, remunerações, honorários pagos por participações em conselhos consultivos, de investigadores, ou outros comitês, etc. Provenientes da indústria farmacêutica, de órteses, próteses, equipamentos e implantes, brasileiras ou estrangeiras:

- Pfizer: Tafamidis; Alnylan: Patisiran.

Fabio Serra Silveira

Declaração financeira

A - Pagamento de qualquer espécie e desde que economicamente apreciáveis, feitos a (i) você, (ii) ao seu cônjuge/ companheiro ou a qualquer outro membro que resida com você, (iii) a qualquer pessoa jurídica em que qualquer destes seja controlador, sócio, acionista ou participante, de forma direta ou indireta, recebimento por palestras, aulas, atuação como proctor de treinamentos, remunerações, honorários pagos por participações em conselhos consultivos, de investigadores, ou outros comitês, etc. Provenientes da indústria farmacêutica, de órteses, próteses, equipamentos e implantes, brasileiras ou estrangeiras:

- Novartis: Entresto; AstraZeneca: Forxiga; Servier: Procoralan.

B - financiamento de pesquisas sob sua responsabilidade direta/pessoal (direcionado ao departamento ou instituição) provenientes da indústria farmacêutica, de órteses, próteses, equipamentos e implantes, brasileiras ou estrangeiras:

- Amgen: Omecantiv mecarbil

Outros relacionamentos

Financiamento de atividades de educação médica continuada, incluindo viagens, hospedagens e inscrições para congressos e cursos, provenientes da indústria farmacêutica, de órteses, próteses, equipamentos e implantes, brasileiras ou estrangeiras:

- Ache: Hipertensão

Felix José Alvarez Ramires Declaração financeira

A - Pagamento de qualquer espécie e desde que economicamente apreciáveis, feitos a (i) você, (ii) ao seu cônjuge/ companheiro ou a qualquer outro membro que resida com você, (iii) a qualquer pessoa jurídica em que qualquer destes seja controlador, sócio, acionista ou participante, de forma direta ou indireta, recebimento por palestras, aulas, atuação como proctor de treinamentos, remunerações, honorários pagos por participações em conselhos consultivos, de investigadores, ou outros comitês, etc. Provenientes da indústria farmacêutica, de órteses, próteses, equipamentos e implantes, brasileiras ou estrangeiras:

- Novartis: Sacubitril/Valsartana; Pfizer: Patisiran; Merck: Vericiquat; Amgen.

\begin{tabular}{ll}
\hline Fernando Antibas Atik & Nada a ser declarado \\
\hline Fernando Bacal & Nada a ser declarado \\
\hline Flávio de Souza Brito & Declaração financeira \\
& A - Pagamento de qualquer espécie e desde que economicamente apreciáveis, feitos a (i) você, (ii) ao seu cônjuge/ \\
& companheiro ou a qualquer outro membro que resida com você, (iii) a qualquer pessoa jurídica em que qualquer \\
& destes seja controlador, sócio, acionista ou participante, de forma direta ou indireta, recebimento por palestras, \\
& aulas, atuação como proctor de treinamentos, remunerações, honorários pagos por participações em conselhos \\
& consultivos, de investigadores, ou outros comitês, etc. Provenientes da indústria farmacêutica, de órteses, próteses, \\
& equipamentos e implantes, brasileiras ou estrangeiras: \\
- Novartis: Entresto; Servier: Procoralan; Merck: Concor.
\end{tabular}

Germano Emílio Conceição Souza Declaração financeira

A - Pagamento de qualquer espécie e desde que economicamente apreciáveis, feitos a (i) você, (ii) ao seu cônjuge/ companheiro ou a qualquer outro membro que resida com você, (iii) a qualquer pessoa jurídica em que qualquer destes seja controlador, sócio, acionista ou participante, de forma direta ou indireta, recebimento por palestras, aulas, atuação como proctor de treinamentos, remunerações, honorários pagos por participações em conselhos consultivos, de investigadores, ou outros comitês, etc. Provenientes da indústria farmacêutica, de órteses, próteses, equipamentos e implantes, brasileiras ou estrangeiras:

- Novartis: Insuficiência Cardíaca; Merck: Insuficiência Cardíaca.

B - Financiamento de pesquisas sob sua responsabilidade direta/pessoal (direcionado ao departamento ou instituição) provenientes da indústria farmacêutica, de órteses, próteses, equipamentos e implantes, brasileiras ou estrangeiras: - Bayer: Rivaroxabana;

Outros relacionamentos

Participação societária de qualquer natureza e qualquer valor economicamente apreciável de empresas na área de saúde, de ensino ou em empresas concorrentes ou fornecedoras da SBC:

- Área da saúde: Prestação de serviços PJ médica, área assistencial

- Ensino: Empresa de ensino técnico para aulas e cursos na área de saúde 

companheiro ou a qualquer outro membro que resida com você, (iii) a qualquer pessoa jurídica em que qualquer destes seja controlador, sócio, acionista ou participante, de forma direta ou indireta, recebimento por palestras, aulas, atuação como proctor de treinamentos, remunerações, honorários pagos por participações em conselhos consultivos, de investigadores, ou outros comitês, etc. Provenientes da indústria farmacêutica, de órteses, próteses, equipamentos e implantes, brasileiras ou estrangeiras:

- Novartis: Insuficiência Cardíaca; Roche: Biomarcadores; Servier: Insuficiência Cardíaca.

C - Financiamento de pesquisa (pessoal), cujas receitas tenham sido provenientes da indústria farmacêutica, de órteses, próteses, equipamentos e implantes, brasileiras ou estrangeiras:

- Roche: GDF-15.

\begin{tabular}{ll}
\hline Jefferson Luis Vieira & Nada a ser declarado \\
\hline João David de Souza Neto & Nada a ser declarado \\
\hline João Manoel Rossi Neto & Declaração financeira
\end{tabular}

A - Pagamento de qualquer espécie e desde que economicamente apreciáveis, feitos a (i) você, (ii) ao seu cônjuge/ companheiro ou a qualquer outro membro que resida com você, (iii) a qualquer pessoa jurídica em que qualquer destes seja controlador, sócio, acionista ou participante, de forma direta ou indireta, recebimento por palestras, aulas, atuação como proctor de treinamentos, remunerações, honorários pagos por participações em conselhos consultivos, de investigadores, ou outros comitês, etc. Provenientes da indústria farmacêutica, de órteses, próteses, equipamentos e implantes, brasileiras ou estrangeiras:

- Novartis: Aulas; AstraZeneca: Aulas.

José Albuquerque de Figueiredo Neto
Declaração financeira

A - Pagamento de qualquer espécie e desde que economicamente apreciáveis, feitos a (i) você, (ii) ao seu cônjuge/ companheiro ou a qualquer outro membro que resida com você, (iii) a qualquer pessoa jurídica em que qualquer destes seja controlador, sócio, acionista ou participante, de forma direta ou indireta, recebimento por palestras, aulas, atuação como proctor de treinamentos, remunerações, honorários pagos por participações em conselhos consultivos, de investigadores, ou outros comitês, etc. Provenientes da indústria farmacêutica, de órteses, próteses, equipamentos e implantes, brasileiras ou estrangeiras:

- Novartis: Insuficiência Cardíaca.

Lídia Ana Zytynski Moura

\section{Declaração financeira}

A - Pagamento de qualquer espécie e desde que economicamente apreciáveis, feitos a (i) você, (ii) ao seu cônjuge/ companheiro ou a qualquer outro membro que resida com você, (iii) a qualquer pessoa jurídica em que qualquer destes seja controlador, sócio, acionista ou participante, de forma direta ou indireta, recebimento por palestras, aulas, atuação como proctor de treinamentos, remunerações, honorários pagos por participações em conselhos consultivos, de investigadores, ou outros comitês, etc. Provenientes da indústria farmacêutica, de órteses, próteses, equipamentos e implantes, brasileiras ou estrangeiras:

- Novartis: Entresto; AstraZeneca: Forxiga.

B - Financiamento de pesquisas sob sua responsabilidade direta/pessoal (direcionado ao departamento ou instituição) provenientes da indústria farmacêutica, de órteses, próteses, equipamentos e implantes, brasileiras ou estrangeiras: - AstraZeneca: Forxiga.

Livia Adams Goldraich

Luís Beck-da-Silva

Nada a ser declarado

Declaração financeira

A - Pagamento de qualquer espécie e desde que economicamente apreciáveis, feitos a (i) você, (ii) ao seu cônjuge/ companheiro ou a qualquer outro membro que resida com você, (iii) a qualquer pessoa jurídica em que qualquer destes seja controlador, sócio, acionista ou participante, de forma direta ou indireta, recebimento por palestras, aulas, atuação como proctor de treinamentos, remunerações, honorários pagos por participações em conselhos consultivos, de investigadores, ou outros comitês, etc. Provenientes da indústria farmacêutica, de órteses, próteses, equipamentos e implantes, brasileiras ou estrangeiras:

- Novartis: Insuficiência Cardíaca; AstraZeneca: Insuficiência Cardíaca.

B - Financiamento de pesquisas sob sua responsabilidade direta/pessoal (direcionado ao departamento ou instituição) provenientes da indústria farmacêutica, de órteses, próteses, equipamentos e implantes, brasileiras ou estrangeiras: - Amgen: Insuficiência Cardíaca

Luis Eduardo Rohde Declaração financeira

A - Pagamento de qualquer espécie e desde que economicamente apreciáveis, feitos a (i) você, (ii) ao seu cônjuge/ companheiro ou a qualquer outro membro que resida com você, (iii) a qualquer pessoa jurídica em que qualquer destes seja controlador, sócio, acionista ou participante, de forma direta ou indireta, recebimento por palestras, aulas, atuação como proctor de treinamentos, remunerações, honorários pagos por participações em conselhos consultivos, de investigadores, ou outros comitês, etc. Provenientes da indústria farmacêutica, de órteses, próteses, equipamentos e implantes, brasileiras ou estrangeiras:

- AstraZeneca: Dapaglifozina; Novartis: Sacubitril-Valsartana; Amgen: Omecamtiv Mecarbil; Merck; Bayer. 


\section{Atualização}

\begin{tabular}{ll}
\hline Luiz Claudio Danzmann & Declaração financeira \\
& A - Pagamento de qualquer espécie e desde que economicamente apreciáveis, feitos a (i) você, (ii) ao seu cônjuge/ \\
& companheiro ou a qualquer outro membro que resida com você, (iii) a qualquer pessoa jurídica em que qualquer \\
& destes seja controlador, sócio, acionista ou participante, de forma direta ou indireta, recebimento por palestras, \\
& aulas, atuação como proctor de treinamentos, remunerações, honorários pagos por participações em conselhos \\
& consultivos, de investigadores, ou outros comitês, etc. Provenientes da indústria farmacêutica, de órteses, próteses, \\
& equipamentos e implantes, brasileiras ou estrangeiras: \\
& - Novartis: Entresto; AstraZeneca: Forxiga; Servier: Procoralan. \\
\hline Manoel Fernandes Canesin & Nada a ser declarado \\
\hline Marcelo Imbroinise Bittencourt & Declaração financeira \\
& A - Pagamento de qualquer espécie e desde que economicamente apreciáveis, feitos a (i) você, (ii) ao seu cônjuge/ \\
& companheiro ou a qualquer outro membro que resida com você, (iii) a qualquer pessoa juridica em que qualquer \\
& destes seja controlador, sócio, acionista ou participante, de forma direta ou indireta, recebimento por palestras, \\
& aulas, atuação como proctor de treinamentos, remunerações, honorários pagos por participações em conselhos \\
& consultivos, de investigadores, ou outros comitês, etc. Provenientes da indústria farmacêutica, de órteses, próteses, \\
& equipamentos e implantes, brasileiras ou estrangeiras: \\
- Geneone - dasa: testes genéticos; Sanofi: Terapia de reposição enzimática; AstraZeneca: Forxiga.
\end{tabular}

\begin{tabular}{ll}
\hline Marcelo Westerlund Montera & Nada a ser declarado \\
\hline Marcely Gimenes Bonatto & Declaração financeira \\
& A - Pagamento de qualquer espécie e desde que economicamente apreciáveis, feitos a (i) você, (ii) ao seu cônjuge/
\end{tabular}
companheiro ou a qualquer outro membro que resida com você, (iii) a qualquer pessoa jurídica em que qualquer destes seja controlador, sócio, acionista ou participante, de forma direta ou indireta, recebimento por palestras, aulas, atuação como proctor de treinamentos, remunerações, honorários pagos por participações em conselhos consultivos, de investigadores, ou outros comitês, etc. Provenientes da indústria farmacêutica, de órteses, próteses, equipamentos e implantes, brasileiras ou estrangeiras: - Novartis: Sacubitril/Nalsartana; AstraZeneca: Forxiga.

Marcus Vinícius Simões Declaração financeira

A - Pagamento de qualquer espécie e desde que economicamente apreciáveis, feitos a (i) você, (ii) ao seu cônjuge/ companheiro ou a qualquer outro membro que resida com você, (iii) a qualquer pessoa jurídica em que qualquer destes seja controlador, sócio, acionista ou participante, de forma direta ou indireta, recebimento por palestras, aulas, atuação como proctor de treinamentos, remunerações, honorários pagos por participações em conselhos consultivos, de investigadores, ou outros comitês, etc. Provenientes da indústria farmacêutica, de órteses, próteses, equipamentos e implantes, brasileiras ou estrangeiras:

- Novartis: entresto; AstraZeneca: Dapagliflozina.

B - Financiamento de pesquisas sob sua responsabilidade direta/pessoal (direcionado ao departamento ou instituição) provenientes da indústria farmacêutica, de órteses, próteses, equipamentos e implantes, brasileiras ou estrangeiras: - Amgen: Omecamtiv/Mecarbil; Beringher Ingelheim: Empagliflozina.

Maria da Consolação Vieira Moreira Nada a ser declarado

Miguel Morita Fernandes-Silva Declaração financeira

A - Pagamento de qualquer espécie e desde que economicamente apreciáveis, feitos a (i) você, (ii) ao seu cônjuge/ companheiro ou a qualquer outro membro que resida com você, (iii) a qualquer pessoa jurídica em que qualquer destes seja controlador, sócio, acionista ou participante, de forma direta ou indireta, recebimento por palestras, aulas, atuação como proctor de treinamentos, remunerações, honorários pagos por participações em conselhos consultivos, de investigadores, ou outros comitês, etc. Provenientes da indústria farmacêutica, de órteses, próteses, equipamentos e implantes, brasileiras ou estrangeiras:

- Novartis: Insuficiência Cardíaca

C - Financiamento de pesquisa (pessoal), cujas receitas tenham sido provenientes da indústria farmacêutica, de órteses, próteses, equipamentos e implantes, brasileiras ou estrangeiras:

- Amgen: Omecamtiv/Insuficiência Cardiaca; Beringher Ingelheim: Empagliflozina.

\begin{tabular}{ll}
\hline Mônica Samuel Avila & Nada a ser declarado \\
\hline Mucio Tavares de Oliveira Junior & Declaração financeira \\
& B - Financiamento de pesquisas sob sua responsabilidade direta/pessoal (direcionado ao departamento ou \\
& instituição) provenientes da indústria farmacêutica, de órteses, próteses, equipamentos e implantes, brasileiras ou \\
& estrangeiras: \\
& - Torrent do Brasil: Droga em desenvolvimento; Sanofi Pasteur: Vacina para gripe. \\
\hline Nadine Clausell & Nada a ser declarado \\
\hline Odilson Marcos Silvestre & Nada a ser declarado \\
\hline
\end{tabular}



companheiro ou a qualquer outro membro que resida com você, (iii) a qualquer pessoa jurídica em que qualquer destes seja controlador, sócio, acionista ou participante, de forma direta ou indireta, recebimento por palestras, aulas, atuação como proctor de treinamentos, remunerações, honorários pagos por participações em conselhos consultivos, de investigadores, ou outros comitês, etc. Provenientes da indústria farmacêutica, de órteses, próteses, equipamentos e implantes, brasileiras ou estrangeiras:

- Pfizer: Amiloidose cardíaca; Alnylam: Amiloidose cardíaca; AstraZeneca: Insuficiência Cardíaca; Novartis.

B - Financiamento de pesquisas sob sua responsabilidade direta/pessoal (direcionado ao departamento ou instituição) provenientes da indústria farmacêutica, de órteses, próteses, equipamentos e implantes, brasileiras ou estrangeiras: - Pfizer: Amiloidose cardíaca.

Outros relacionamentos

Financiamento de atividades de educação médica continuada, incluindo viagens, hospedagens e inscrições para congressos e cursos, provenientes da indústria farmacêutica, de órteses, próteses, equipamentos e implantes, brasileiras ou estrangeiras:

- AstraZeneca: Insuficiência Cardíaca; Pfizer: Insuficiência Cardíaca.

Pedro Vellosa Schwartzmann Declaração financeira

A - Pagamento de qualquer espécie e desde que economicamente apreciáveis, feitos a (i) você, (ii) ao seu cônjuge/ companheiro ou a qualquer outro membro que resida com você, (iii) a qualquer pessoa jurídica em que qualquer destes seja controlador, sócio, acionista ou participante, de forma direta ou indireta, recebimento por palestras, aulas, atuação como proctor de treinamentos, remunerações, honorários pagos por participações em conselhos consultivos, de investigadores, ou outros comitês, etc. Provenientes da indústria farmacêutica, de órteses, próteses, equipamentos e implantes, brasileiras ou estrangeiras:

- Novartis: Entresto; Servier: Ivabradina; AstraZeneca: Dapagliflozina; Merck: Serono.

B - Financiamento de pesquisas sob sua responsabilidade direta/pessoal (direcionado ao departamento ou instituição) provenientes da indústria farmacêutica, de órteses, próteses, equipamentos e implantes, brasileiras ou estrangeiras: - Novartis: investigacional; Eidos: AG10.

Outros relacionamentos

Financiamento de atividades de educação médica continuada, incluindo viagens, hospedagens e inscrições para congressos e cursos, provenientes da indústria farmacêutica, de órteses, próteses, equipamentos e implantes, brasileiras ou estrangeiras:

- Bayer: Rivaroxaban; AstraZeneca: Dapagliflozina.

Reinaldo Bulgarelli Bestetti

Nada a ser declarado

Ricardo Mourilhe-Rocha Declaração financeira

A - Pagamento de qualquer espécie e desde que economicamente apreciáveis, feitos a (i) você, (ii) ao seu cônjuge/ companheiro ou a qualquer outro membro que resida com você, (iii) a qualquer pessoa jurídica em que qualquer destes seja controlador, sócio, acionista ou participante, de forma direta ou indireta, recebimento por palestras, aulas, atuação como proctor de treinamentos, remunerações, honorários pagos por participações em conselhos consultivos, de investigadores, ou outros comitês, etc. Provenientes da indústria farmacêutica, de órteses, próteses, equipamentos e implantes, brasileiras ou estrangeiras:

- AstraZeneca: Dapagliflozina; Boehringer: Empagliflozina; Novartis: SacubitrilNalsartana.

B - Financiamento de pesquisas sob sua responsabilidade direta/pessoal (direcionado ao departamento ou instituição) provenientes da indústria farmacêutica, de órteses, próteses, equipamentos e implantes, brasileiras ou estrangeiras: - PROADI/SUS: Telemedicina; Boehringer: Empagliflozina.

\begin{tabular}{ll}
\hline Sabrina Bernadez-Pereira & Nada a ser declarado \\
\hline Salvador Rassi & Declaração financeira
\end{tabular}

A - Pagamento de qualquer espécie e desde que economicamente apreciáveis, feitos a (i) você, (ii) ao seu cônjuge/ companheiro ou a qualquer outro membro que resida com você, (iii) a qualquer pessoa jurídica em que qualquer destes seja controlador, sócio, acionista ou participante, de forma direta ou indireta, recebimento por palestras, aulas, atuação como proctor de treinamentos, remunerações, honorários pagos por participações em conselhos consultivos, de investigadores, ou outros comitês, etc. Provenientes da indústria farmacêutica, de órteses, próteses, equipamentos e implantes, brasileiras ou estrangeiras:

- Novartis: Entresto; Servier: Procoralan.

B - Financiamento de pesquisas sob sua responsabilidade direta/pessoal (direcionado ao departamento ou instituição) provenientes da indústria farmacêutica, de órteses, próteses, equipamentos e implantes, brasileiras ou estrangeiras: - Novartis: Entresto; Servier: Procoralan; Boehringer Ingelheim: Jardiance.

Outros relacionamentos

Financiamento de atividades de educação médica continuada, incluindo viagens, hospedagens e inscrições para congressos e cursos, provenientes da indústria farmacêutica, de órteses, próteses, equipamentos e implantes, brasileiras ou estrangeiras:

- Novartis: Entresto; Servier: Procoralan. 


\section{Atualização}

\begin{tabular}{ll}
\hline Sandrigo Mangini & Declaração financeira \\
& A - Pagamento de qualquer espécie e desde que economicamente apreciáveis, feitos a (i) você, (ii) ao seu cônjuge/ \\
& companheiro ou a qualquer outro membro que resida com você, (iii) a qualquer pessoa jurídica em que qualquer \\
& destes seja controlador, sócio, acionista ou participante, de forma direta ou indireta, recebimento por palestras, \\
& aulas, atuação como proctor de treinamentos, remunerações, honorários pagos por participações em conselhos \\
& consultivos, de investigadores, ou outros comitês, etc. Provenientes da indústria farmacêutica, de órteses, próteses, \\
& equipamentos e implantes, brasileiras ou estrangeiras: \\
& - Novartis: Sacubitril/Valsartan; Pfizer: Doenças raras \\
& Outros relacionamentos \\
& Financiamento de atividades de educação médica continuada, incluindo viagens, hospedagens e inscrições para \\
& congressos e cursos, provenientes da indústria farmacêutica, de órteses, próteses, equipamentos e implantes, \\
& brasileiras ou estrangeiras: \\
& - Pfizer: Doenças raras \\
& Nada a ser declarado \\
\hline Silvia Marinho Martins Alves & Declaração financeira \\
\hline Silvia Moreira Ayub Ferreira & compananento de qualquer espécie e desde que economicamente apreciáveis, feitos a (i) você, (ii) ao seu cônjuge/ \\
& destes seja controlador, sócio, acionista ou participante, de forma direta ou indireta, recebimento por palestras, \\
& aulas, atuação como proctor de treinamentos, remunerações, honorários pagos por participações em conselhos \\
& consultivos, de investigadores, ou outros comitês, etc. Provenientes da indústria farmacêutica, de órteses, próteses, \\
& equipamentos e implantes, brasileiras ou estrangeiras: \\
- Abbott: Mitraclip; Novartis: Entresto. \\
Outros relacionamentos \\
Financiamento de atividades de educação médica continuada, incluindo viagens, hospedagens e inscrições para \\
congressos e cursos, provenientes da indústria farmacêutica, de órteses, próteses, equipamentos e implantes, \\
brasileiras ou estrangeiras: \\
- Abbott: Heartmate Il e HeartMate 3. \\
Nada a ser declarado \\
\hline Victor Sarli Issa
\end{tabular}

